\title{
Semi-group Conditions for Affine Algebraic Plane Curves with More than One Place at Infinity
}

\author{
Penelope G. WIGHTWICK \\ Department of Mathematics and Statistics \\ University of Melbourne \\ Victoria 3010 - Australia \\ P.Wightwick@ms . unimelb.edu . au
}

Received: July 7, 2005

Accepted: September 28, 2006

\begin{abstract}
An interesting and open question is the classification of affine algebraic plane curves. Abhyankar and Moh [1] completely described the possible links at infinity for those curves where the link has just one component, a knot. Such curves are said to have one place at infinity. The Abhyankar-Moh result has been of great assistance in classifying those polynomials which define a connected curve with one place at infinity. This paper provides a new proof of the Abhyankar-Moh result which is then used to find a description for the case where the polynomial defines a curve with one point at infinity.

Key words: affine algebraic curves, classification, link at infinity.

2000 Mathematics Subject Classification: 14R05 (14H20).
\end{abstract}

\section{Introduction}

An important topological feature of curves defined by polynomials is the link at infinity. For a polynomial $f \in \mathbb{C}[x, y]$ the link at infinity is the intersection of the associated curve $C$, defined by $f(x, y)=0$, with a three dimensional sphere in $\mathbb{C}^{2}$ of radius large enough that all isolated singularities of $C$ lie within the sphere. The link at infinity is invariant under automorphisms of $\mathbb{C}^{2}$ and is an important tool for

This research was supported by an Australian Postgraduate Award. 
studying the topology of such curves. Consider the fibres, $\left\{f^{-1}(c): c \in \mathbb{C}\right\}$, of a polynomial $f \in \mathbb{C}[x, y]$. A fibre $f^{-1}(c)$ is regular if for some neighbourhood $D$ of $c \in \mathbb{C}$, $\left.f\right|_{f^{-1}(D)}: f^{-1}(D) \rightarrow D$ is a locally trivial $C^{\infty}$ fibration. Thus $f^{-1}(c)$ looks like nearby fibres and has no isolated singularities. The link at infinity of a regular plane curve $C$ determines the topology of $C \subset \mathbb{C}^{2}$ as an embedded smooth 2-manifold up to ambient isotopy (see [18]). If $C$ is not regular, the link at infinity, together with the Milnor numbers of any singularities in $C$, gives the Euler characteristic of $C$. Thus knowledge of the link at infinity of regular algebraic plane curves helps one to classify those with a given topology.

The question of classification of polynomials up to equivalence under automorphisms of $\mathbb{C}^{2}$ for certain specific cases has been addressed by a number of mathematicians. Perhaps the most well-known case is the Abhyankar-Moh-Suzuki theorem $[1,28]$ which says that, if $f \in \mathbb{C}[x, y]$ defines a curve $f(x, y)=0$ that is smooth, connected and contractible, then $f$ is equivalent to $g(x, y)=x$. This theorem has been proved by a number of people. As well as proving the theorem in a new way, Artal Bartolo [6] gives a history of proofs of this result.

Various people have looked at the classification of those polynomials which define a curve which is connected with one puncture, that is, where the link at infinity is a knot. Such polynomials are said to have one place at infinity. Neumann [18] has given a classification up to equivalence of irreducible polynomials whose curves are smooth, once punctured, connected and of genus less than or equal to 4. Miyanishi [16] describes simple forms to which once punctured, irreducible, smooth affine curves of genera 1, 2, 3, 4 are equivalent. A'Campo and Oka [5] have classified smooth curves of genus 1 or 2 with one place at infinity. Nakazawa and Oka [17] classify smooth, algebraic plane curves with one place at infinity of given genus $\leq 16$ and have written down all possible normal forms of equations. There has been some duplication of cases classified, however the techniques used have varied from researcher to researcher.

Another related result is that of Zaidenberg and Lin [33] who have classified curves which are contractible and singular.

Progress in classification has also been made for polynomials for which every fibre is smooth and connected but not contractible. Artal Bartolo, Cassou-Noguès, and Velasco [8] have looked at families of smooth polynomial mappings of degree $6 n+4$ with fibres that are irreducible and generically of genus $n$. Cassou-Noguès [11] has used rational maps to find other families of polynomials with fibres that are smooth and irreducible.

A beautiful result of Abhyankar and Moh ([1]) completely described the possible links at infinity for those curves with one place at infinity. This result has been of great assistance in classifying those polynomials which define a connected curve with one place at infinity.

Given the importance of the Abhyankar-Moh result it is natural to ask

Can we find descriptions of the links at infinity for affine algebraic plane curves which have more than one place at infinity? 
This question is addressed in sections 3 and 4 and descriptions are found for two cases, in particular for the case of one point at infinity and many places at infinity.

To describe links at infinity of affine algebraic plane curves we use the splice diagrams developed by Eisenbud and Neumann in [12]. These are a particularly useful way of encoding the details of certain links. Briefly the splice diagram is a finite tree with certain numerical and other decorations on it. These splice diagrams are particularly useful in the analysis of the links of affine algebraic plane curves. In section 2 we give a description of the splice diagram as it applies to the link at infinity of an algebraic curve and how to find it. This was first described by Neumann in [18] and also by Cassou-Noguès in [10] and Wightwick in [31]. We refer the reader to [18] and [10] for a full discussion of the properties of splice diagrams and the invariants which arise from these.

The splice diagram encoding the information for the link at infinity for a curve with one place at infinity is

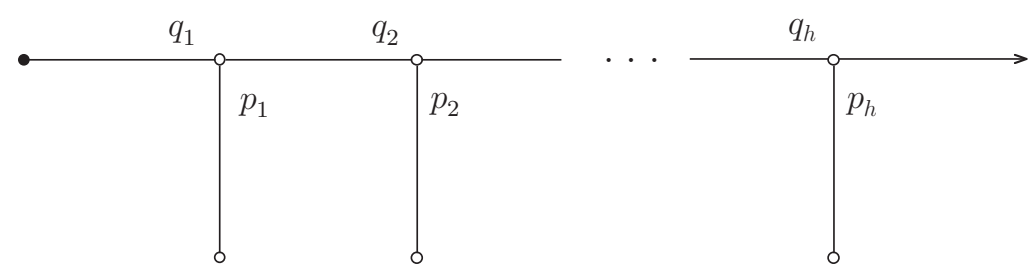

with $\operatorname{gcd}\left(p_{k}, q_{k}\right)=1, p_{k}, q_{k}>0$, for $k=1,2, \ldots, h$. The conditions, called the Abhyankar-Moh semi-group conditions, are defined as follows. Let

$$
\text { semi-group }\left\{\delta_{0}, \delta_{1}, \ldots, \delta_{j-1}\right\}
$$

be the set of all non-zero linear combinations of $\delta_{0}, \delta_{1}, \ldots, \delta_{j-1}$ with non-negative integer coefficients.

Theorem 1.1 ([1]). Let $f \in \mathbb{C}[x, y]$ be an irreducible polynomial that defines a curve $f(x, y)=0$ with one place at infinity with the above splice diagram. Then for $j=1,2,3, \ldots, h$,

$$
\begin{aligned}
q_{j} p_{j} p_{j+1} & \cdots p_{h} \\
& \in \operatorname{semi-group}\left\{p_{1} p_{2} \cdots p_{h}, q_{1} p_{2} p_{3} \cdots p_{h}, q_{2} p_{3} p_{4} \cdots p_{h}, \ldots, q_{j-1} p_{j} \cdots p_{h}\right\} .
\end{aligned}
$$

Sathaye ([24]) and Sathaye and Stenerson ([25]) show that any set of the linking numbers as defined above, $\left\{p_{1}, q_{1}, p_{2}, q_{2}, \ldots, p_{h}, q_{h}\right\}$, satisfying the Abhyankar-Moh semi-group conditions is realisable as a semi-group associated with an algebraic curve.

In section 3 a new elementary proof for the Abhyankar-Moh result is provided. This uses the Newton polygons that are used in finding the Newton-Puiseux expansion of the polynomial. The Newton-Puiseux expansion refers to finding the roots of a 
polynomial, $f(x, y)$ in the form $y-a(x)$ where $a$ is a fractional power series in $x^{1 / n}$ for some positive integer $n$ (or similarly in the form $x-a(y)$ ). The advantage of the new proof is that it can easily be extended to find semi-group conditions which hold in other special cases.

The Newton polygons used in this paper are based on those used in [31] and differ from those described in [18] and [10]. As the form of the Newton polygons used to find the Newton-Puiseux expansion of a polynomial is a key element of the proof of the Abhyankar-Moh result, we give, in section 2, a detailed description of the procedure that is used.

In the original proof of Theorem 1.1 Abhyankar and Moh used the theory of valuations. Suzuki ([28]) has given an alternative proof of this result using the theory of resolutions. His proof gives some valuable insight into the geometry of an algebraic curve with one place at infinity. Another readable proof was given by Pinkham ([22]).

In section 4, I generalize the semi-group conditions from the case of one place at infinity to the important case of polynomials which define curves which have one point at infinity. The semi-group conditions arise quite naturally from the shapes of the splice diagrams (see sections 3 and 4 ).

Assuming that we have $f \in \mathbb{C}[x, y]$ irreducible with one point at infinity occurring at $y=0$, then $f$ will be monic in $y$ but may have more than one component in the link at infinity. In this case there will be a maximal $h$ such that the splice diagram of $f$ contains the following section:

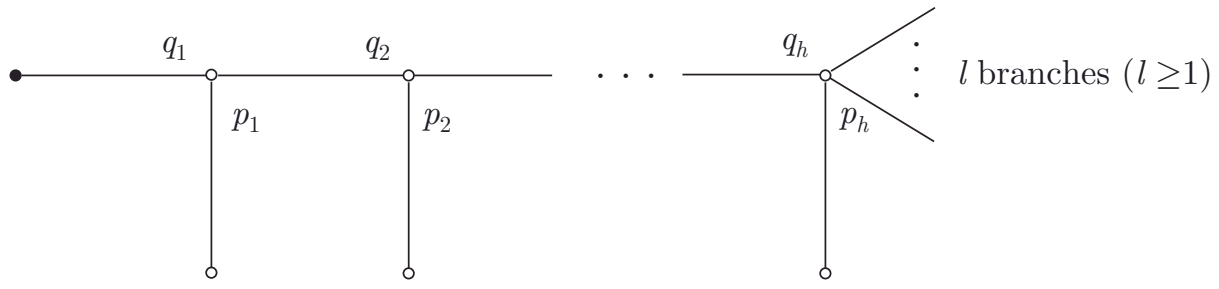

and such that $\operatorname{deg}_{x}(f)<\operatorname{deg}_{y}(f)=p_{1} p_{2} \cdots p_{h} d$ for some positive integer $d$.

Theorem 1.2. Let $f \in \mathbb{C}[x, y]$ be an irreducible polynomial that defines a curve $f(x, y)=0$ with one point at infinity with its splice diagram containing the section pictured above.

Then for $j=1,2, \ldots, h-1$,

$$
\begin{aligned}
& q_{j} p_{j} \cdots p_{h} d \\
& \quad \in \text { semi-group }\left\{p_{1} p_{2} \cdots p_{h} d, q_{1} p_{2} p_{3} \cdots p_{h} d, q_{2} p_{3} p_{4} \cdots p_{h} d, \ldots, q_{j-1} p_{j} \cdots p_{h} d\right\} .
\end{aligned}
$$

Furthermore, there exists $r \in \mathbb{Z}$ with $1 \leq r \leq l$ such that

$$
r q_{h} d \in \text { semi-group }\left\{p_{1} p_{2} \cdots p_{h} d, q_{1} p_{2} p_{3} \cdots p_{h} d, q_{2} p_{3} p_{4} \cdots p_{h} d, \ldots, q_{h-1} p_{h} d\right\} .
$$


Here the Abhyankar-Moh semi-group conditions are the case $d=1$.

Note that the knots that are components of links at infinity are classified — they are simply the knots whose Puiseux pairs satisfy the reverse Puiseux inequalities (see [18]). Thus any additional restriction on Puiseux pairs of a branch must depend on how different branches are positioned with respect to each other. Moreover, examples show that one really only expects to be able to get a reasonable condition on an individual branch up to the point where branches diverge; weights no longer even have to be positive, in fact it is not hard to make them arbitrarily negative beyond this point.

The proof of Theorem 1.2 also gives some restrictions on the case with two points at infinity, but these are rather more technical (see section 5).

Ultimately one would like to understand what splice diagrams may be realised as affine algebraic plane curves and to understand the moduli spaces of the families of polynomials which give rise to these. The results and methods in this paper may help to achieve this objective.

\section{Iterated torus links and the splice diagram}

In this section we describe the splice diagrams of links at infinity of affine algebraic plane curves, and how to use the method of Newton polygons to find these. Some parts of this section have been discussed in [20].

\subsection{The splice diagram}

The link at infinity of an algebraic plane curve is a cabled torus link in $S^{3}$ and can be conveniently represented by the splice diagram. The splice diagram is a tree with various decorations. We briefly describe splice diagrams below. See Neumann [18], and Cassou-Noguès [10] for further details. For example, if the link at infinity is a knot it has a splice diagram of the form

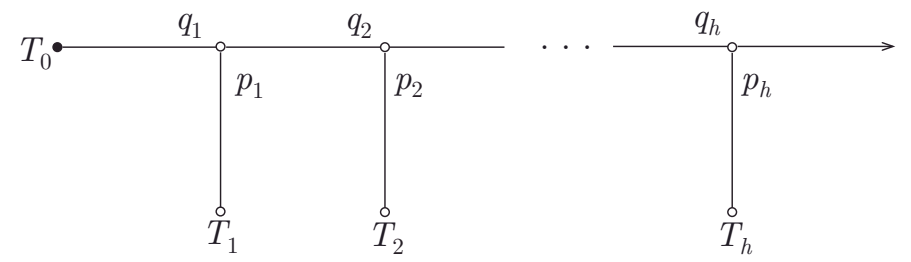


This splice diagram encodes the information that we:

- Start with an unknot.

- Cable a $\left(p_{1}, q_{1}\right)$ torus knot on the unknot.

- Cable a $\left(p_{2}, q_{2}\right)$ torus knot on the $\left(p_{1}, q_{1}\right)$ torus knot.

- Cable a $\left(p_{h}, q_{h}\right)$ torus knot on the $\left(p_{h-1}, q_{h-1}\right)$ torus knot.

The final $\left(p_{h}, q_{h}\right)$ cable is the link at infinity. The earlier "virtual" components used in constructing the link are represented by the vertices, $T_{1}, \ldots, T_{h}$ (those with just one edge adjoining them), the unknot being represented by the node $T_{1}$ and the $\left(p_{k}, q_{k}\right)$ cable being represented by $T_{k+1}$. The nodes $T_{0}$ and $T_{1}$ form a virtual Hopf link. Note that

- $T_{0}$ is called the "root node" and is indicated by a solid disk rather than a circle.

- The valency of a vertex is the number of edges adjoining it.

- Vertices of valency 1 other than the root node are called leaves.

- Vertices of valency 2 or more are called nodes.

- Arrowheads represent actual components of the link at infinity.

- The weights attached to the diagram are oriented with respect to the root node, the weight closest to the root node on an edge is called the near weight and the one furthest away (if it exists) is called a far weight. Edge weights of 1 are usually omitted from the splice diagram.

- We can associate a numeric weight with each component of a link. Such a link, together with the numeric weights is called a multilink. The numeric weights are included in splice diagrams at the end of the arrowheads corresponding to the components. Such weights occur quite naturally in algebraic links and reflect the multiplicity of factors of polynomials. 
Let $f \in \mathbb{C}[x, y]$ be such that

$$
f=f_{N}+f_{N-1}+\cdots+f_{1}+f_{0}
$$

where each $f_{i}$ is homogeneous of degree $i$. Then the points at infinity where the algebraic plane curve $f^{-1}(c)$ meet infinity are the solutions of

$$
f_{N}(x, y)=0 .
$$

If there is more than one point at infinity the link at infinity is constructed by cabling on a Hopf link with one component for each point at infinity. There may be nodes of valency greater than three. For example

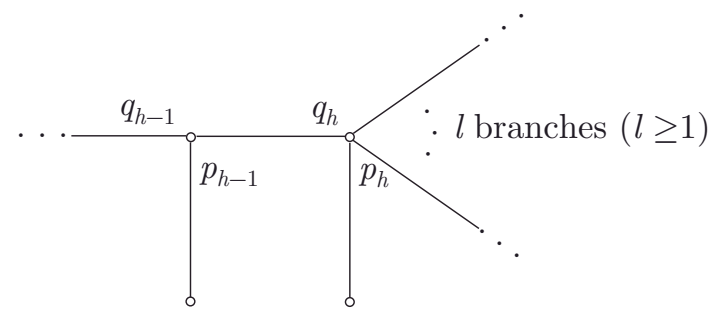

may arise in the construction of the link. In this case there are $l$ parallel $\left(p_{h}, q_{h}\right)$ torus knots cabled on the $\left(p_{h-1}, q_{h-1}\right)$ cable.

If we take $c \in \mathbb{C}$ then an algebraic curve, $f^{-1}(c)$, is regular if for some neighbourhood of $c$, say $D,\left.f\right|_{f^{-1}(D)}$ is a locally trivial fibration. For all but finitely many values of $c, f^{-1}(c)$ is regular.

A minimal Seifert surface, $F$ say, is a Seifert surface with maximum Euler characteristic among all oriented embedded surfaces with no closed components in $S^{3}$ with $\partial F=\mathcal{L}$ where $\mathcal{L}$ is a link. $F$ may not be unique up to isotopy as a Seifert surface and the isotopy need not fix $\mathcal{L}$.

Theorem 2.1 ([18]). The topology of a regular algebraic curve $V=f^{-1}(c) \subset \mathbb{C}^{2}$, as an embedded smooth manifold is determined up to ambient isotopy by its link $\mathcal{L}$ at infinity. In fact a minimal Seifert surface $F$ for $\mathcal{L}$ in $S^{3}$ is unique up to isotopy in $S^{3}$, and $V$ can be recovered up to isotopy by attaching a collar out to infinity in $\mathbb{C}^{2}$ to the boundary of $F$.

While we are able to recover a minimal Seifert surface with the same topology as a regular algebraic curve this is not the case for irregular curves. However we are at least able to find the Euler characteristic for this case.

Not all cable links arise as the link at infinity of an algebraic plane curve. The splice diagrams of links at infinity of algebraic plane curves can always be reduced to a unique minimal splice diagram satisfying the following conditions:

(i) All near weights are positive.

(ii) At most one near weight of a node differs from 1 . 
(iii) For the following edge in a splice diagram

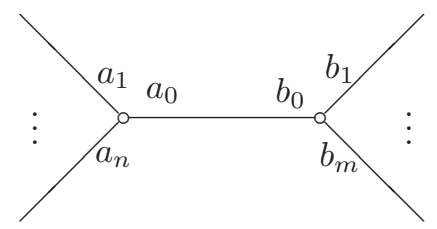

the edge determinant of the edge is

$$
a_{0} b_{0}-a_{1} \cdots a_{n} b_{1} \cdots b_{m} .
$$

All the edge determinants of a splice diagram for a link at infinity are negative.

A non-minimal splice diagram of a link at infinity can be simplified by the following moves:

EN 1: removing edges of weight 1 ending in a leaf or

EN 2: removing nodes of valency 2 (other than the root node).

We call a diagram which does not admit either of these moves the minimal splice diagram of $f$. If we allow deletion of the root vertex we obtain the minimal unrooted splice diagram of $f$.

We can use the above moves to reduce the number of edges in a splice diagram.

Theorem $2.2([12,18])$. If $\Gamma$ is a splice diagram which does not contain any edges of weight one ending in a leaf or nodes of valency 2 then $\Gamma$ is minimal. Moreover $\Gamma$ is unique in its equivalence class up to change of sign.

Note that Theorem 2.2 implies that if we remove the root vertex from a splice diagram and obtain a minimal splice diagram satisfying its conditions then the resultant splice diagram is a combinatorial invariant of a given link at infinity of an algebraic plane curve under automorphisms of $\mathbb{C}^{2}$. However the same is not true for the rooted splice diagram which includes additional information about the curve defined by a specific polynomial. For example, the root node encodes the number of points at infinity of the curve and the degree of the polynomial (see below).

\subsection{The Newton-Puiseux expansion and the link at infinity}

The Newton-Puiseux techniques for studying the singularities of affine algebraic plane curves have been long established (for example see $[14,21,23]$ ). It is only relatively recently that these methods have been extended to include the study of points at infinity (see Abhyankar and Moh [1], Neumann [18] and Cassou-Noguès [10]). 
The idea is to find the Puiseux expansion for each component of the link at infinity. For example, if a component of a link at infinity corresponds to the curve meeting infinity at $y=0$ we obtain an equation of the form

$$
y=x^{q_{1} / p_{1}}\left(t_{1}+x^{-\tau_{2} /\left(p_{1} p_{2}\right)}\left(t_{2}+x^{-\tau_{3} /\left(p_{1} p_{2} p_{3}\right)}+\cdots\right)\right)
$$

with $q_{1}<p_{1}, \operatorname{gcd}\left(p_{1}, q_{1}\right)=1, p_{i}>0, \tau_{i}>0$, and $\operatorname{gcd}\left(p_{i}, \tau_{i}\right)=1$ for all $i$. For some $h, p_{j}=1$ for all $j>h$. We need only find the terms up to this point. This gives a branch of the splice diagram for $f=0$ corresponding to one component of the link at infinity:

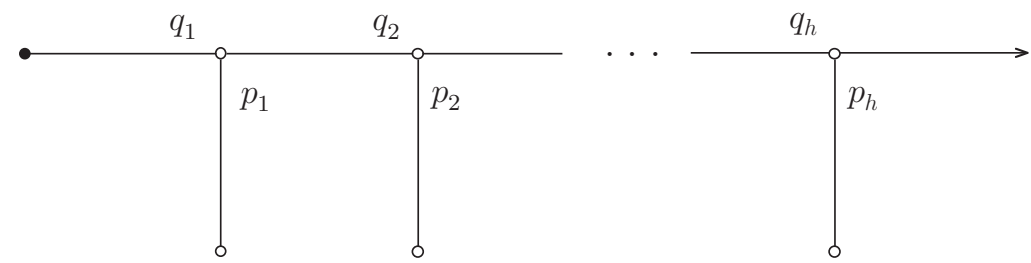

where $q_{i}=q_{i-1} p_{i-1} p_{i}-\tau_{i}$ for $i=2, \ldots, h$. In this context "branch of the splice diagram" means part of the finite tree in the natural way.

Definition 2.3. The pairs $\left(p_{1}, q_{1}\right),\left(p_{2},-\tau_{2}\right), \ldots,\left(p_{h},-\tau_{h}\right)$ are called Newton pairs. The pairs $\left(p_{1}, q_{1}\right),\left(p_{2}, q_{2}\right), \ldots,\left(p_{h}, q_{h}\right)$ are called cabling pairs.

Note that one or more of the $p_{i}$ may be equal to 1 . Replacing a link component by a $\left(1, q_{i}\right)$ cable does not change the topology of the link. We can find all the relevant equations and assemble the branches of the splice diagram by merging parts that correspond to identical initial Puiseux expansions.

In practice we use Newton polygons to find the Newton and cabling pairs and build up the splice diagram.

Definition 2.4. Let $f \in \mathbb{C}\left[x^{-1}, x, y\right]$ where

$$
f=\sum a_{\alpha \beta} x^{\alpha} y^{\beta}
$$

and consider the curve $C$ defined by $f(x, y)=0$. The Newton polygon of $f$ is the convex hull of the points

$$
\left\{(\alpha, \beta) \mid a_{\alpha \beta} \neq 0\right\} \text {. }
$$

The Newton polygon of $f$ is denoted $\operatorname{NP}(f)$.

The process of using Newton polygons to find the splice diagram is as follows: 


\section{SteP 1: Find the Starting Hopf LinK}

$f=f_{N}+f_{N-1}+\cdots+f_{0}$ where $f_{i}$ is homogeneous of degree $i$. We find the solutions of $f_{N}=0$, which will be of the form $x=a, y=b$ or $y=c x, a \neq 0$. We can always apply linear automorphisms of $\mathbb{C}[x, y]$ of the form $\phi(x, y)=\left(c_{1} x+d_{1} y, c_{2} x+d_{2} y\right)$ to ensure that the roots will be one of the following:

(i) Where there is just one point at infinity: $y=0$

(ii) Where there are two point at infinity: $y=0$ and $x=0$

(iii) Where there are more than 2, say $r+2$, points at infinity: $y=0, x=0$ and $y=c_{i} x, i=1, \ldots, r$.

The initial splice diagram will consist of a root node and one arrowhead for each point at infinity. In two special cases we stop at this point.

(i) If $f=(y-b)^{N}$, the Newton polygon includes the point $(0, N)$ which corresponds to one point at infinity. The link is the unknot with multiplicity $N$.

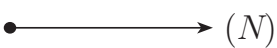

(ii) If $f=(x-a)^{m}(y-b)^{n}$ with $m, n \geq 1$, the Newton polygon includes the point $(m, n)$, not on either axis, and the points at infinity are $x=0$ and $y=0$. The link is the Hopf link with two components with multiplicity $m$ and $n$ respectively.

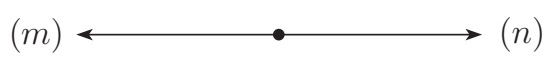

There are two other special cases worthy of mention.

(iii) If $f=\prod_{i=1}^{m}\left(y-a_{i}\right)^{n_{i}}, m>1$ then there is one point at infinity and $m$ components of the link at infinity. The solutions to $f(x, y)=0$ are $y=a_{i} x^{0 / 1}$ with multiplicity $n_{i}$. We replace the one component Hopf link (the unknot) by $m$ parallel $(0,1)$ cables giving $m$ parallel unknots.

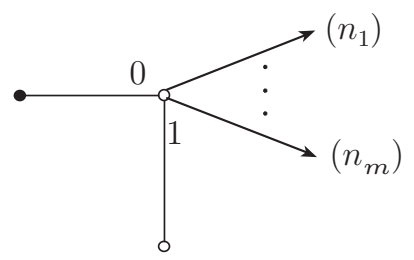


(iv) If $f=x^{a} y^{b} \prod_{i=1}^{m}\left(x^{q} y^{p}-c_{i}\right)^{n_{i}}$ then there are two points at infinity, $x=0$ and $y=0$. We can see that the fractional power series solutions are of the form

$$
y=\sqrt[p]{c_{i}} x^{-q / p}
$$

or

$$
x=\sqrt[q]{c_{i}} x^{-p / q}
$$

In addition we may have solutions $y=0$ or $x=0$ with multiplicity $b$ and $a$ respective. Thus we have cabling pairs $(p, q)$ for the $y=0$ point at infinity and $(q, p)$ for the $x=0$ point at infinity. We obtain the splice diagram

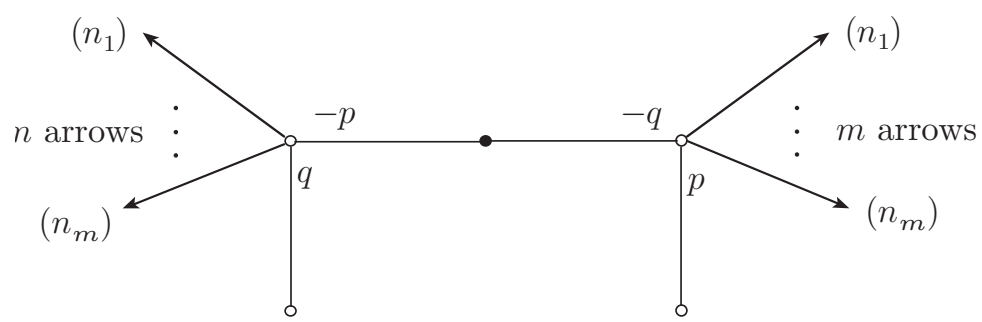

if $a=b=0$; otherwise for $a>0$ or $b>0$ we retain the original component of the Hopf link with the relevant multiplicity $a$ or $b$.

From now on we assume that we need to add one or more cables to the initial Hopf link. To analyse these cases we will need the following definition.

Definition 2.5. Let

$$
f=\sum_{\alpha, \beta} a_{\alpha \beta} x^{\alpha} y^{\beta} .
$$

The $(u, v)$ weighted degree of $a_{\alpha \beta} x^{\alpha} y^{\beta}, a_{\alpha \beta} \neq 0$ is $u \alpha+v \beta$. The maximum $(u, v)$ weighted degree of $f$ is

$$
\max \left\{u \alpha+v \beta \mid a_{\alpha \beta} \neq 0\right\}
$$

Express $f$ in the form

$$
f=\sum_{u \alpha+v \beta=d} a_{\alpha \beta} x^{\alpha} y^{\beta}+\sum_{u \alpha+v \beta<d} a_{\alpha \beta} x^{\alpha} y^{\beta}
$$

where $d$ is the maximum $(u, v)$ weighted degree of $f$. Then the terms in

$$
\sum_{u \alpha+v \beta=d} a_{\alpha \beta} x^{\alpha} y^{\beta}
$$

lie in the boundary of $\operatorname{NP}(f)$. If there are two or more such terms then there is a line segment in $\partial \operatorname{NP}(f)$ satisfying $u \alpha+v \beta=d$. 
Definition 2.6. A single point in $\partial \mathrm{NP}(f)$ satisfying $u \alpha+v \beta=d$ is called a maximum $(u, v)$ point of $\mathrm{NP}(f)$ while a line segment consisting of all points satisfying $u \alpha+v \beta=$ $d$ is called the maximum $(u, v)$ line segment of $\mathrm{NP}(f)$.

We then have the following:

(i) The line segments corresponding to the point at infinity $y=0$ are the maximum $\left(p_{i}, q_{i}\right)$ line segments of $\mathrm{NP}(f), i=1, \ldots, m$ say, where $p_{i}, q_{i} \in \mathbb{Z}, p_{i}>0$, $\operatorname{gcd}\left(p_{i}, q_{i}\right)=1$ and $p_{i}>q_{i}$. These line segments have slope $-p_{i} / q_{i}$, although one slope might be infinite. For convenience we think of this as slope $1 / 0$.

(ii) The line segments corresponding to the point at infinity $x=0$ are the maximum $\left(q_{j}^{\prime}, p_{j}^{\prime}\right)$ line segments of $\mathrm{NP}(f), j=1, \ldots, k$ say, where $q_{j}^{\prime}, p_{j}^{\prime} \in \mathbb{Z}, p_{j}^{\prime}>0$, $\operatorname{gcd}\left(p_{j}^{\prime}, q_{j}^{\prime}\right)=1$ and $p_{j}^{\prime}>q_{j}^{\prime}$. These line segments have slope $-q_{j}^{\prime} / p_{j}^{\prime}$.

(iii) The line segment in the Newton polygon which corresponds to points at infinity of the form $y=a x, a \neq 0$, if any, is the maximum $(1,1)$ line segment of $\mathrm{NP}(f)$, a line segment of slope -1 .

The following diagram shows the relevant line segments in $\partial \mathrm{NP}(f)$ if there are three or more points at infinity:

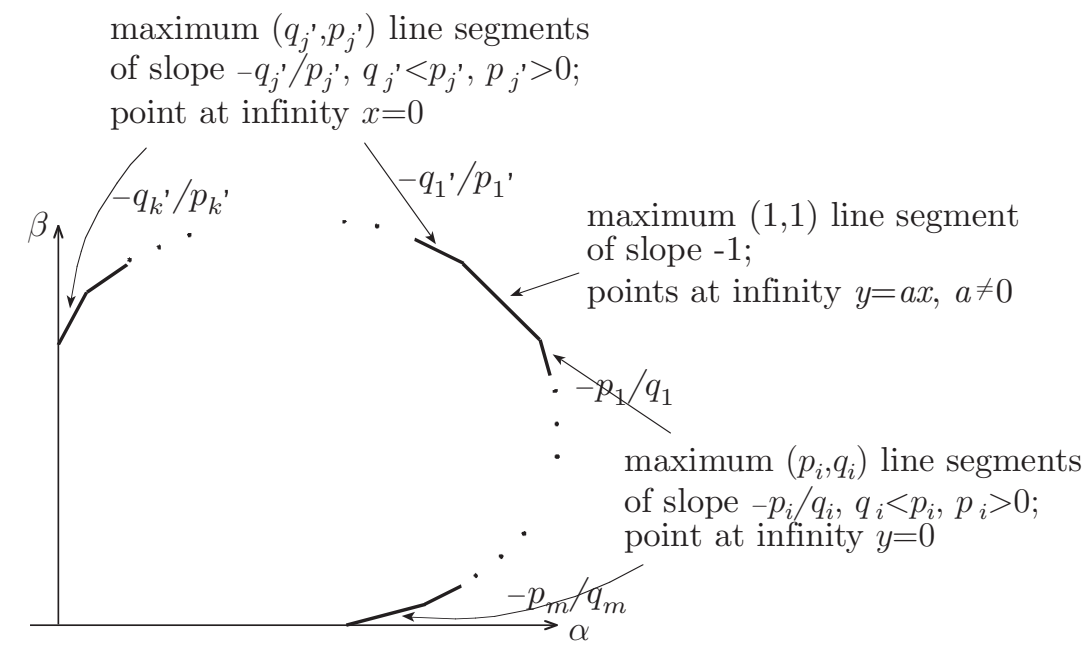

We will describe the procedure for finding further cabling pairs for the point at infinity $y=0$. For the point at infinity, $x=0$, one reverses the role of $x$ and $y$ in the initial substitution. For a point at infinity $y=a x$ we first use an affine automorphism of $\mathbb{R}^{2}$, say $\phi:(x, y) \mapsto\left(c_{1} x+c_{2} y, d_{1} x+d_{2} y\right)$ to move the point at infinity to $y=0$ and work with $\phi \circ f$ from Step 2 . 


\section{SteP 2: AdDing the FiRst CABLeS}

For each maximum $\left(p_{i}, q_{i}\right)$ line segment of $\mathrm{NP}(f)$ corresponding to the point at infinity $y=0$ we consider the corresponding terms in $f$ :

$$
\sum_{p_{i} \alpha-q_{i} \beta=d_{i}} a_{\alpha \beta} x^{\alpha} y^{\beta}=c_{i} x^{a_{i}} y^{b_{i}} \prod_{j=1}^{s_{i}}\left(y^{p_{i}}-t_{i j}^{p_{i}} x^{q_{i}}\right)^{l_{i j}} .
$$

This gives $s_{i}$ solutions

$$
y=t_{i j} x^{q_{i} / p_{i}}
$$

where $p_{i}>0, q_{i}<p_{i}$ and a Newton pair (and cabling pair), $\left(p_{i}, q_{i}\right)$. Note that if two solutions differ by a root of unity they are equivalent. We have $q_{1} / p_{1}>\cdots>q_{m} / p_{m}$. We add each $\left(p_{i}, q_{i}\right)$ cable to the $y=0$ component of the Hopf link in turn. At the $i^{\text {th }}$ operation we retain the Hopf link component unless it is the $m^{\text {th }}$ operation in which case we only retain the original Hopf component if $f$ has a factor $y^{b}, b \neq 0$. For the case where $y^{b}$ is not a factor of $f$ we obtain

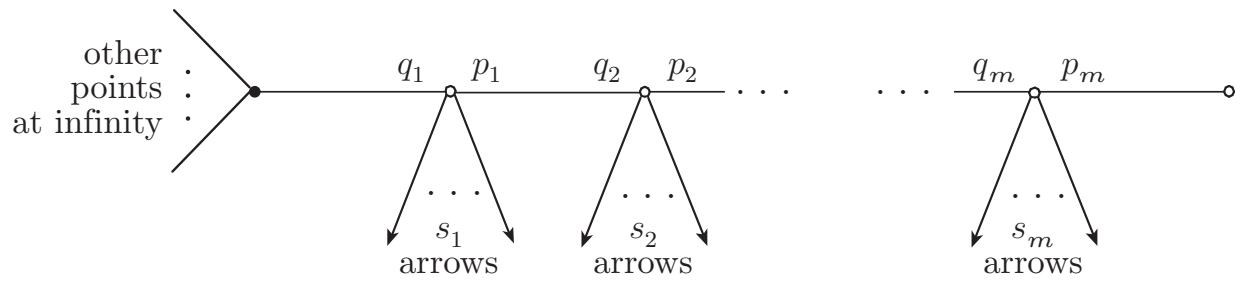

For the case where $y^{b}$ is a factor of $f$ we obtain

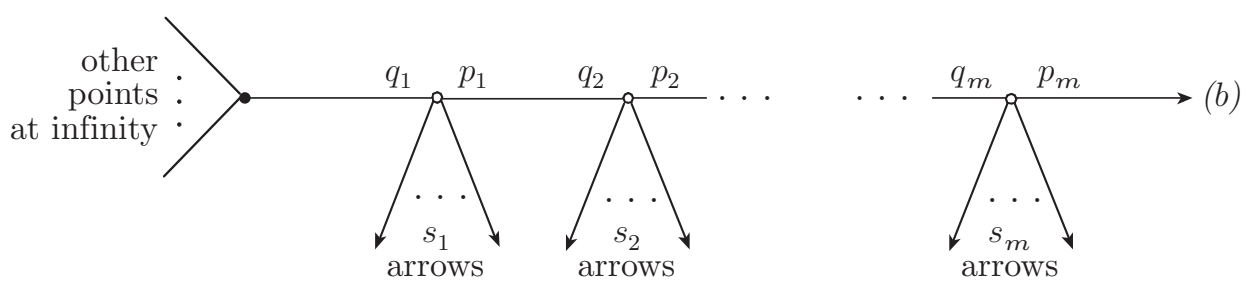

Thus we obtain $s_{i}$ arrowheads for each maximum $\left(p_{i}, q_{i}\right)$ line segment. If any $l_{i j}=1$ no further cabling occurs on the relevant branch of the splice diagram and the corresponding arrowhead represents an actual component of the link at infinity. Otherwise we continue as in Step 3.

\section{Step 3: Further CABling}

For each solution $y=t_{i j} x^{q_{i} / p_{i}}$ where $l_{i j}>1$ we make the substitution

$$
x=x_{1}^{p_{i}} \text { and } y=x_{1}^{q_{i}}\left(y_{1}+t_{i j}\right)
$$


to find $f_{1}\left(x_{1}, y_{1}\right) \in \mathbb{C}\left[x_{1}^{-1}, x_{1}, y_{1}\right]$. Draw $\mathrm{NP}\left(f_{1}\right)$. Then the line segments corresponding to branches at infinity are the maximum $\left(p_{i j, k},-\tau_{i j, k}\right)$ line segments of $\operatorname{NP}\left(f_{1}\right)$ where $p_{i j, k}, \tau_{i j, k}>0$ and $p_{i j, k}, \tau_{i j, k} \in \mathbb{Z}, k=1, \ldots, m_{i j}$.

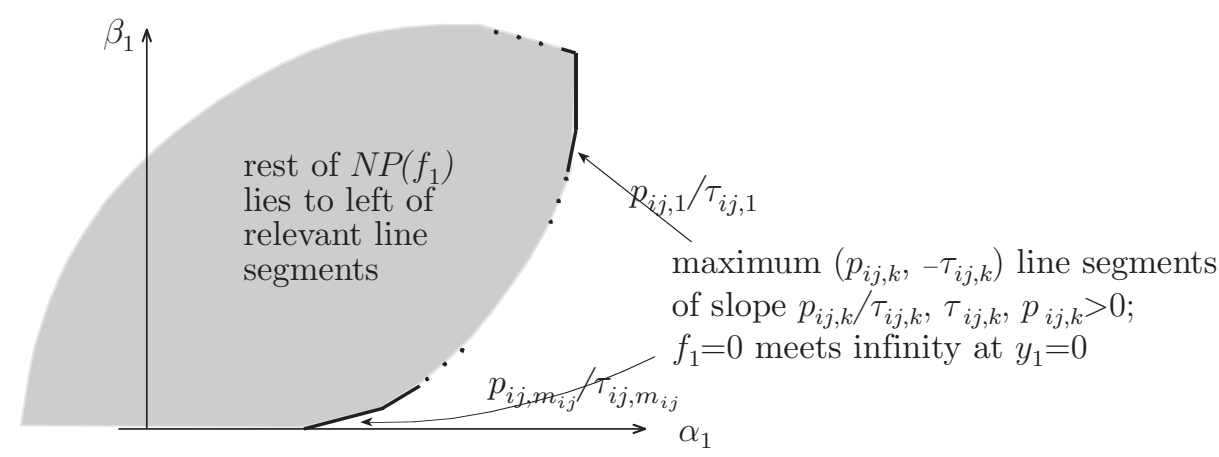

If there is just one line segment of height one so that the only Newton pair is $\left(p_{i j, 1},-\tau_{i j, 1}\right)$ with $p_{i j, k}=1$ we stop cabling on the branch. Otherwise, we analyse each line segment as in Step 2. We obtain Newton pairs

$$
\left(p_{i j, 1},-\tau_{i j, 1}\right), \ldots,\left(p_{i j, m_{i j}},-\tau_{i j, m_{i j}}\right)
$$

giving cabling pairs

$$
\begin{aligned}
\left(p_{i j, 1}, q_{i} p_{i} p_{i j, 1}-\tau_{i j, 1}\right), \ldots,\left(p_{i j, m_{i j}}, q_{i} p_{i} p_{i j, m_{i j}}\right. & \left.-\tau_{i j, m_{i j}}\right) \\
& =\left(p_{i j, 1}, q_{i j, 1}\right), \ldots,\left(p_{i j, m_{i j}}, q_{i j, m_{i j}}\right)
\end{aligned}
$$

and

$$
q_{i j, 1} / p_{i j, 1}>\cdots>q_{i j, m_{i j}} / p_{i j, m_{i j}} .
$$

Cabling gives that the $(i j)^{\text {th }}$ arrowhead is replaced by the shaded region:

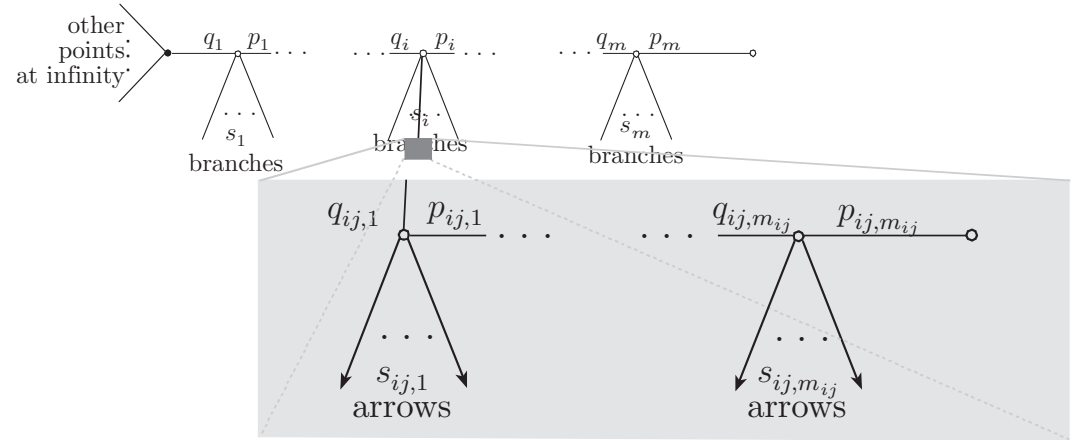


if $f_{1}$ does not have a factor $y_{1}^{b_{i j}}$ for some $b_{i j} \neq 0$, otherwise the original component is retained and we obtain

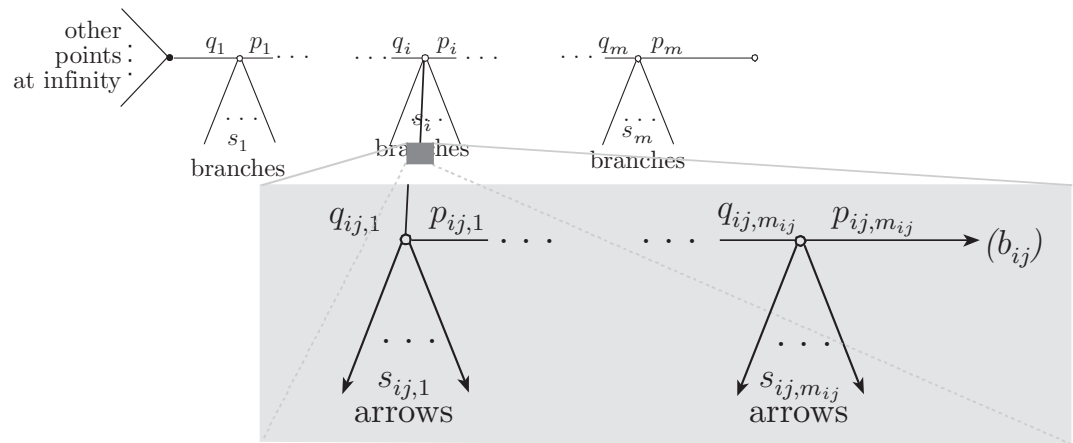

Let the maximum $\left(p_{i j, k},-\tau_{i j, k}\right)$ line segment have associated terms

$$
d_{k} x_{1}^{a_{k}} y_{1}^{b_{k}} \prod_{r=1}^{s_{i j, k}}\left(y_{1}^{p_{i j, k}}-t_{k, r}^{p_{i j, k}} x_{1}^{-\tau_{i j, k}}\right)^{l_{k, r}} .
$$

We add $s_{i j, k}\left(p_{i j, k}, q_{i j, k}\right)$ cables to the $k^{\text {th }}$ component. If any $l_{k, r}=1$ no further cabling will be required on that branch and the arrowhead represents an actual component in the link at infinity for $f$. For $l_{k, r} \neq 1$ we make substitutions of the form

$$
\left(x_{1}, y_{1}\right)=\left(x_{2}^{p_{i j, k}}, x_{2}^{-\tau_{i j, k}}\left(y_{2}+t_{k, r}\right)\right) .
$$

The first two terms of the Newton Puiseux expansion for the $r^{\text {th }}$ solution corresponding to the $k^{\text {th }}$ pair are now known:

$$
y=t_{i j} x^{q_{i} / p_{i}}+t_{k, r} x^{q_{i} / p_{i}} x^{-\tau_{i j, k} /\left(p_{i} p_{i j, k}\right)}+\cdots .
$$

We continue in this fashion to find the Newton pairs. Apart from the initial cabling step, all the Newton polygons will be of the form found in Step 3.

Example 2.7 (The Briançon Polynomial).

$$
f(x, y)=x^{2}(1+x y)^{4}+3 x(1+x y)^{3}+(3-8 / 3 x)(1+x y)^{2}-4(1+x y)+y .
$$

We will find the splice diagram for $f(x, y)=0, \Gamma\left(f^{-1}(0)\right)$. We first find the points at infinity by solving $x^{2}(x y)^{4}=0$ to get two points at infinity, $x=0$ and $y=0$. Thus our initial splice diagram is that of the two component Hopf link. Drawing the Newton polygon, $\mathrm{NP}(f)$, we have 


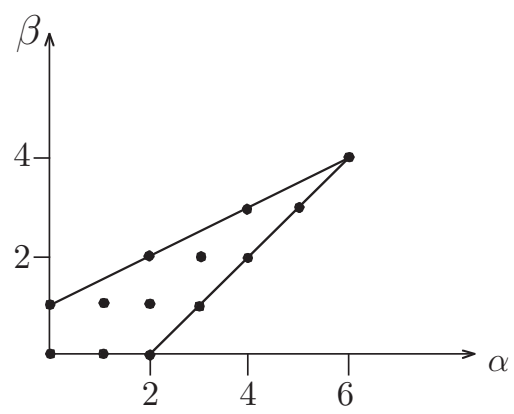

For $y=0$ there is one line segment and the terms in $f$ corresponding to this are

$$
x^{2}(1+x y)^{4} .
$$

We solve $(1+x y)^{4}=0$, obtaining $y=-x^{-1 / 1}$ as a solution. The Newton pair is $(1,-1)$ as is the cabling pair. As the exponent of $(1+x y)^{4}$ is more than 1 we need to continue the algorithm. We substitute $(x, y)=\left(x_{1}^{1}, x_{1}^{-1}\left(y_{1}-1\right)\right)$ to obtain

$$
f_{1}=x_{1}^{2} y_{1}^{4}+3 x_{1} y_{1}^{3}+3 y_{1}^{2}-8 / 3 x_{1} y_{1}^{2}-4 y_{1}+x_{1}^{-1} y_{1}-x_{1}^{-1}
$$

with Newton polygon

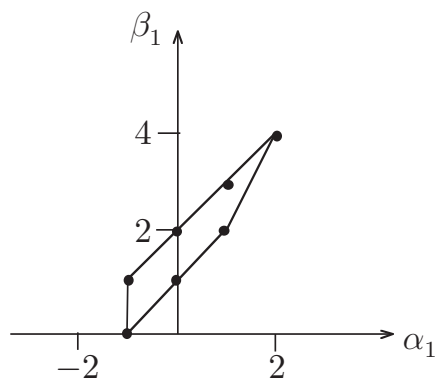

We have two line segments corresponding to branches at infinity. The first, of slope 2, has corresponding terms $f_{1,1}=x_{1}^{2} y_{1}^{4}-8 / 3 x_{1} y_{1}^{2}=x_{1}^{2} y_{1}^{2}\left(y_{1}^{2}-8 / 3 x_{1}^{-1}\right)$ giving a solution $y_{1}=\sqrt[2]{8 / 3} x_{1}^{-1 / 2}$ as the required solution. The Newton pair is $(2,-1)$ and the cabling pair is $(2,-1 \times 1 \times 2-1)=(2,-3)$. As the exponent of $\left(y_{1}^{2}-8 / 3 x_{1}^{-1}\right)$ in $f_{1,1}$ is equal to one, we have completed this branch.

The other line segment of slope 1 has corresponding terms

$$
f_{1,2}=-8 / 3 x_{1} y_{1}^{2}-4 y_{1}-x_{1}^{-1}=-8 / 3 x_{1}\left(y_{1}+(3+\sqrt{3}) / 4 x_{1}^{-1}\right)\left(y_{1}+(3-\sqrt{3}) / 4 x_{1}^{-1}\right)
$$

with two relevant solutions of $f_{1,2}=0$. The Newton pair is $(1,-1)$ and we add two $(1,-1 \times 1 \times 1-1)=(1,-2)$ parallel cables. As the exponents of $\left(y_{1}+(3+\sqrt{3}) / 4 x_{1}^{-1}\right)$ and $\left(y_{1}+(3-\sqrt{3}) / 4 x_{1}^{-1}\right)$ are both equal to 1 in $f_{1,2}$ we have completed this branch. 
For $x=0$ there is again one line segment with the corresponding terms being

$$
y\left(\left(x^{2} y\right)^{3}+3\left(x^{2} y\right)^{2}+3\left(x^{2} y\right)+1\right)=y\left(x^{2} y+1\right)^{3}
$$

with solution $x=-y^{-1 / 2}$. Putting $(x, y)=\left(x_{1}^{-1}\left(y_{1}-1\right), x_{1}^{2}\right)$, we obtain $f_{1}^{\prime}$ which has a Newton polygon with one relevant line segment giving Newton pair $(3,-1)$ and cabling pair $(3,-1 \times 2 \times 3-1)=(3,-7)$ at which stage we stop.

Thus we build up the splice diagrams as follows:

Step 1: Hopf link

Step 3: Next cabling

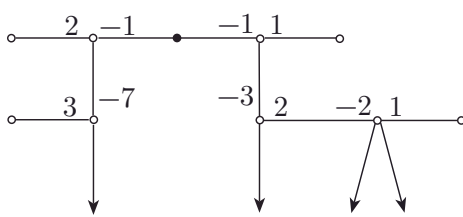

Step 2: First cabling

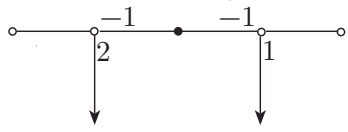

Apply EN1 and EN2

$\approx$

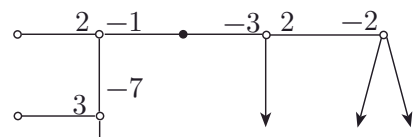

In step 3, one obtains two edges of weight 1 adjoining leaves. The reduced splice diagram was obtained by first deleting theses leaves and edges and then deleting the resultant node of valency two.

\subsection{Invariants from splice diagrams}

Definition 2.8. Given a vertex, $v$, of a splice diagram, $\Gamma$, the linking coefficient at $v$, also called the multiplicity at $v$ is the sum over the arrowheads, $w$, of $\Gamma$ :

$$
l_{v}=\sum_{w} l(v, w)
$$

where $l(v, w)$ is the product of edge weights directly adjacent to, but not on, the path from $v$ to $w$ in $\Gamma$.

The splice diagram for the Briançon polynomial, $\Gamma\left(f^{-1}(0)\right)$ (Example 2.7) has the linking coefficients indicated in brackets in the diagram below: 
(1)

(2)

(10)

$(0) \quad(-1)$

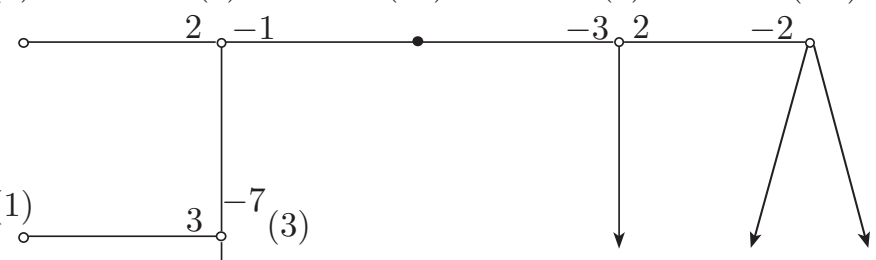

PROPERTIES OF THE LINKING COEFFICIENTS

The properties relating to the linking coefficients of splice diagrams of links at infinity are as follows:

(i) The degree of a polynomial is always the linking coefficient $l_{\bullet}$ at the root vertex.

(ii) A splice diagram is regular, that is, the splice diagram of a regular fibre of $f$, if and only if it has no negative linking coefficients.

(iii) A polynomial has fibres with irregular splice diagrams if and only if the regular splice diagram has at least one zero linking coefficient. Each irregular diagram determines the regular one and the regular one strongly constrains the number and form of the irregular ones. See Neumann ([19]), for details.

(iv) The Euler characteristic of the generic fibre of $f$ is

$$
\chi_{\mathrm{reg}}:=\sum_{v \in \operatorname{vert} \Gamma(f)}\left(2-\sigma_{v}\right) l_{v},
$$

where vert $\Gamma(f)$ is the set of non-arrowhead vertices of $\Gamma(f)$ and $\sigma_{v}$ is the valency of vertex $v$ (number of edges at $v$ ).

(v) For any $c \in \mathbb{C}$ define the Milnor number at infinity of $f^{-1}(c)$ as

$$
\lambda_{c}:=\sum_{\substack{v \in \Gamma\left(f^{-1}(c)\right) \\ l_{v}<0}}\left(2-\sigma_{v}\right) l_{v}
$$

so $\lambda_{c}=0$ unless $\Gamma\left(f^{-1}(c)\right)$ is irregular. Moreover, if $f^{-1}(c)$ is a reduced fibre (that is, one without multiple components), the total Milnor number is the sum of Milnor numbers (see Milnor [15])

$$
\mu_{c}:=\sum_{p \in f^{-1}(c)} \mu_{p} .
$$


As $\mu_{p}=0$ at non-singular points, this is a finite sum. Then the Euler characteristic of a reduced fibre $f^{-1}(c)$ is given by

$$
\chi\left(f^{-1}(c)\right)=\mu_{c}+\sum_{v \in \operatorname{vert} \Gamma\left(f^{-1}(c)\right)}\left(2-\sigma_{v}\right) l_{v}=\mu_{c}+\lambda_{c}+\chi_{\mathrm{reg}} .
$$

(vi) Suzuki ([27]) showed that for any $f$ one has

$$
1-\chi_{\mathrm{reg}}=\mu_{c}+\sum_{c \in \mathbb{C}}\left(\chi\left(f^{-1}(c)\right)-\chi_{\mathrm{reg}}\right)
$$

so, if $f$ has only isolated singularities,

$$
1-\chi_{\mathrm{reg}}=\sum_{c \in \mathbb{C}}\left(\mu_{c}+\lambda_{c}\right)
$$

One consequence of this formula is that, once we have found finite singularities and singularities at infinity contributing enough $\mu_{c}$ and $\lambda_{c}$ to satisfy the formula, we know that we have found all non-generic fibres.

Example 2.9 (The Briançon Polynomial continued). We have found the splice diagram $\Gamma\left(f^{-1}(0)\right)$ for this polynomial. There are two other splice diagrams for links at infinity. For the regular fibres $f^{-1}(t), t \neq 0,-16 / 9$ we find that $\Gamma\left(f^{-1}(t)\right.$ is

(1)

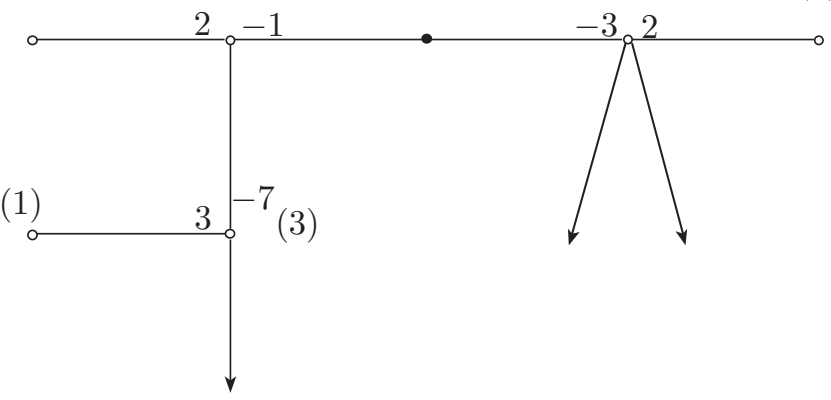


while for the other fibre $f^{-1}(-16 / 9), \Gamma\left(f^{-1}(-16 / 9)\right)$ is

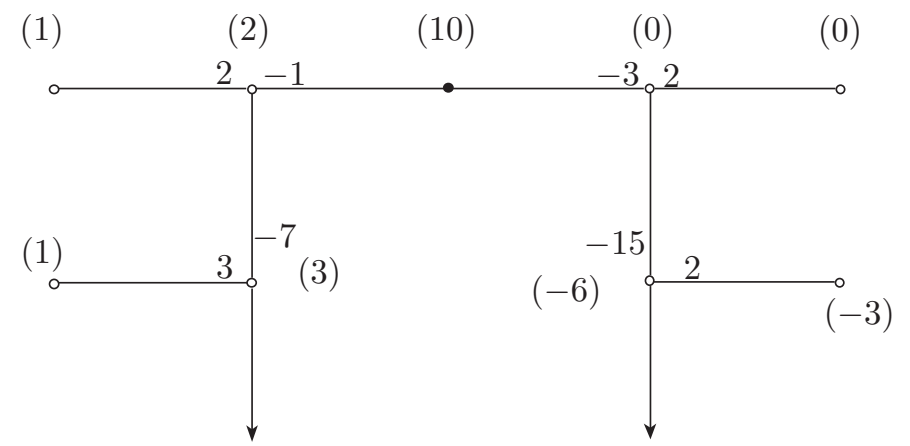

From these diagrams we find

$$
\begin{gathered}
\chi_{\mathrm{reg}}=1(2-1)+3(2-3)+1(2-1)+2(2-3)+10(2-2)+0(2-3)=-3, \\
\lambda_{0}=(-1)(2-3)=1,
\end{gathered}
$$

and

$$
\lambda_{-16 / 9}=3 \text {. }
$$

We thus obtain

$$
\chi_{\mathrm{reg}}+\lambda_{0}+\lambda_{-16 / 9}=-3+1+3=1
$$

so that $\mu_{c}=0$ for all $c$. If follows that the Briançon polynomial has no finite singularities.

\section{The semi-group condition for curves with one place at in- finity}

This section gives the new proof of Theorem 1.1. We start by restating the theorem. Let $f \in \mathbb{C}[x, y]$ be an irreducible polynomial that defines a curve $f(x, y)=0$ with one place at infinity, thus having a link at infinity whose minimal splice diagram is as follows:

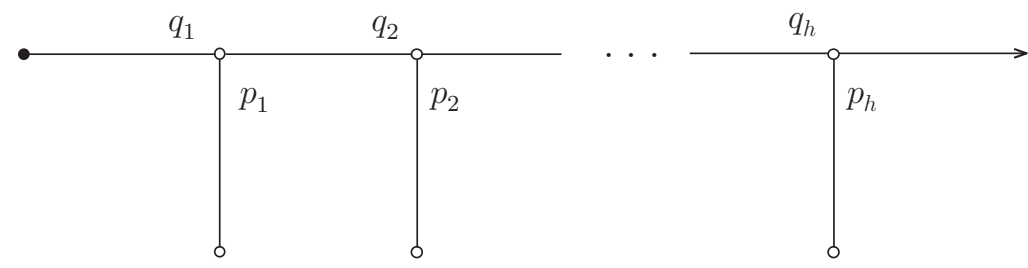

Name the vertices of the splice diagram as follows. Starting from the root vertex and moving towards the arrowhead: 
- vertices with one adjoining edge are $u_{0}, u_{1}, \ldots, u_{h}$

- vertices with three adjoining edges are $v_{1}, v_{2}, \ldots, v_{h}$

Let $L$ denote the link at infinity and let $S_{w}$ denote the virtual component of the link corresponding to the node $w$ for $w \in\left\{u_{i}: i=1, \ldots, h\right\} \cup\left\{v_{j}: 1, \ldots, h\right\}$. Then the linking numbers of the virtual components with the link at infinity are named as follows:

$$
\begin{aligned}
& \delta_{0}=l_{u_{0}}=\operatorname{link}\left(S_{u_{0}}, L\right)=p_{1} p_{2} \cdots p_{h}, \\
& \delta_{1}=l_{u_{1}}=\operatorname{link}\left(S_{u_{1}}, L\right)=q_{1} p_{2} p_{3} \cdots p_{h}, \\
& \delta_{2}=l_{u_{2}}= \operatorname{link}\left(S_{u_{2}}, L\right)=q_{2} p_{3} p_{4} \cdots p_{h}, \\
& \vdots \\
& \delta_{h}=l_{u_{h}}=\operatorname{link}\left(S_{u_{h}}, L\right)=q_{h}, \\
& \psi_{1}=l_{v_{1}}=\operatorname{link}\left(S_{v_{1}}, L\right)=q_{1} p_{1} p_{2} \cdots p_{h}, \\
& \psi_{2}=l_{v_{2}}=\operatorname{link}\left(S_{v_{2}}, L\right)=q_{2} p_{2} p_{3} \cdots p_{h}, \\
& \quad \vdots \\
& \psi_{h}=l_{v_{h}}=\operatorname{link}\left(S_{v_{h}}, L\right)=q_{h} p_{h} .
\end{aligned}
$$

We now restate Theorem 1.1 in terms of these linking numbers.

Theorem 1.1 (The Abhyankar-Moh Semi-Group Theorem restated). Let $f \in \mathbb{C}[x, y]$ be an irreducible polynomial that defines a curve $f(x, y)=0$ with one place at infinity. Then using the above notation we have:

For $j=1,2,3, \ldots, h$,

$$
\psi_{j} \in \operatorname{semi-group}\left\{\delta_{0}, \delta_{1}, \ldots, \delta_{j-1}\right\}
$$

where semi-group $\left\{\delta_{0}, \delta_{1}, \ldots, \delta_{j-1}\right\}$ is the set of all non-zero linear combinations of $\delta_{0}, \delta_{1}, \ldots, \delta_{j-1}$ with non-negative integer coefficients.

This theorem limits the possible values of $q_{j}$, in terms of $p_{1}, \ldots, p_{j-1}$ and $q_{1}, \ldots, q_{j-1}$.

\subsection{The essence of the proof}

The proof of the Theorem 1.1 depends on some rather technical lemmas so, before giving all the details, we describe the main ideas and show how these combine to give the result. In the case of $f=y+c$, Theorem 1.1 is trivially true. We prove the theorem under the assumption that the cabling pairs found are $\left\{\left(p_{1}, q_{1}\right),\left(p_{2}, q_{2}\right)\right.$, $\left.\ldots,\left(p_{h}, q_{h}\right)\right\}$ with $p_{i}>1, i=1, \ldots, h$. The case of redundant cabling pairs of the type $(1, s)$ is addressed in section 3.6 and the proof follows the same lines as the case without redundant pairs.

There are three main steps in the proof. 
(i) We express the polynomial $f$ as the sum of other polynomials using "approximate roots of polynomials" (see below).

(ii) We then analyse the Newton polygon used to find the cabling pair $\left(p_{k}, q_{k}\right)$ by finding the contribution of each summand to the Newton polygon.

(iii) Finally we show that the actual proof follows fairly easily from this analysis.

Step 1: Approximate roots of polynomials (SeCtion 3.2)

Abhyankar and Moh [3] developed the concept of approximate roots of polynomials. Their main result is the following lemma (Lemma 3.7).

Let $f \in \mathbb{C}[x, y]$ be monic in $y$ with $\operatorname{deg}_{y}(f)=k l, k, l \in \mathbb{Z}, k, l>1$. Then there exists a unique $g \in \mathbb{C}[x, y]$ such that $g$ is monic in $y, \operatorname{deg}_{y}(g)=k$ and

$$
f(x, y)=g^{l}+\zeta
$$

where $\operatorname{deg}_{y}(\zeta)<(l-1) k=(l-1) \operatorname{deg}_{y}(g)$.

Definition 3.1. The polynomial $g$ is the $l^{\text {th }}$ approximate root of $f$.

The main result used in the proof of Theorem 1.1 is Lemma 3.9 (Suzuki [28]). Let $f \in \mathbb{C}[x, y]$ be monic in $y$ and $\operatorname{deg}_{y}(f)=p_{1} p_{2} \cdots p_{k} \cdots p_{h}$. Then $f$ can be written as

$$
f=g_{k}^{p_{k} \cdots p_{h}}+\zeta_{k}
$$

where $g_{k}$ is the $\left(p_{k} \cdots p_{h}\right)^{t h}$ approximate root of $f$. Moreover

$$
\zeta_{k}=\sum_{\gamma_{0}, \ldots, \gamma_{k}} a_{\gamma_{0} \ldots \gamma_{k}} g_{0}^{\gamma_{0}} g_{1}^{\gamma_{1}} \cdots g_{k}^{\gamma_{k}}
$$

where $g_{0}=x$ and $g_{1}, g_{2}, \ldots, g_{k-1}$ are the $\left(p_{1} p_{2} \cdots p_{h}\right)^{\text {th }},\left(p_{2} \cdots p_{h}\right)^{\text {th }}, \ldots$, $\left(p_{k-1} \cdots p_{h}\right)^{\text {th }}$ approximate roots of $f$ respectively. In addition, $0 \leq \gamma_{0}, 0 \leq \gamma_{1}<p_{1}$, $\ldots, 0 \leq \gamma_{k-1}<p_{k-1}, 0 \leq \gamma_{k}<p_{k} \cdots p_{h}-1$.

We use the approximate roots where $p_{1}, p_{2}, \ldots, p_{h}$ are given in the splice diagram of $f$.

Step 2: The Newton polygons of $f, g_{0}, \ldots, g_{h}$ (SeCtions 3.3 And 3.5 )

We match features in the " $k$ th" Newton polygon of $f, \mathrm{NP}_{k}(f)$ say, used to find the pair $\left(p_{k}, q_{k}\right)$ with the $k^{\text {th }}$ Newton polygons of the components of $\zeta_{k}, a_{\gamma_{0} \ldots \gamma_{k}} g_{0}^{\gamma_{0}} g_{1}^{\gamma_{1}} \cdots g_{k}^{\gamma_{k}}$. Before doing so we give a few definitions.

Definition 3.2. The line segments in the boundary of a Newton polygon which correspond to the points at infinity of a curve $C$ defined by $f(x, y)=0$ where $f \in \mathbb{C}[x, y]$ are described in section 2. These line segments will be called the key line segments of the Newton polygon. 
Definition 3.3. If

$$
f(x, y)=\sum a_{\alpha \beta} x^{\alpha} y^{\beta}
$$

then the points in the set

$$
\left\{(\alpha, \beta) \mid a_{\alpha \beta} \neq 0\right\}
$$

are called the key points of $\mathrm{NP}(f)$, the Newton polygon of $f$.

We define the notion of corner point where there is one or no key line segments in a Newton polygon.

Definition 3.4. Let $\left\{(\alpha, \beta) \mid a_{\alpha \beta} \neq 0\right\}$ be the set of key points of $\operatorname{NP}(f)$. Then the corner point of $\mathrm{NP}(f)$ is the following key point:

(i) where there is a key line segment, the point $(\alpha, \beta)$ in the key line segment such that $\beta$ is maximum.

(ii) where there is no key line segment, the point $\left(\alpha^{\prime}, \beta^{\prime}\right)$ such that $\alpha^{\prime}=\max \{\alpha \mid$ $\left.a_{\alpha \beta} \neq 0\right\}$ and $\beta^{\prime}=\min \left\{\beta\left|\left(\alpha^{\prime}, \beta\right)\right| a_{\alpha^{\prime} \beta} \neq 0\right\}$.

(1)

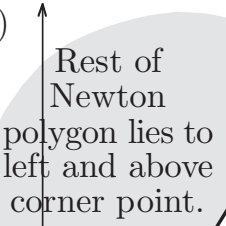

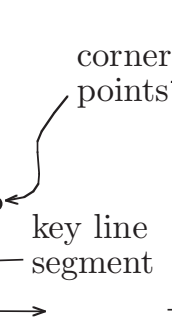

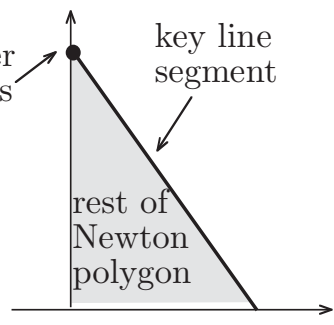

(2)

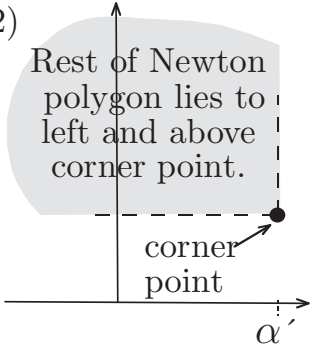

Some relevant facts about Newton polygons are proved in section 3.4.

We now turn to the Newton polygons of $f$. Without loss of generality we assume that $C$ meets infinity at $y=0$. Assume that in order to find the Newton pairs of $f$ we make the substitutions:

$$
\Phi_{j}:\left(x_{j}, y_{j}\right)=\left(x_{j+1}^{p_{j}}, x_{j+1}^{-\tau_{j}}\left(y_{j+1}+t_{j}\right)\right)
$$

for $j=1, \ldots, h-1$, where $\left(x_{1}, y_{1}\right)=(x, y)$.

Definition 3.5. Let

$$
f_{j}=f \circ \Phi_{1} \circ \cdots \circ \Phi_{j-1}
$$

for $j=2, \ldots, h$. Denote the Newton polygons, $\mathrm{NP}(f), \operatorname{NP}\left(f_{2}\right), \ldots, \operatorname{NP}\left(f_{h}\right)$ by

$$
\mathrm{NP}_{1}(f), \mathrm{NP}_{2}(f), \ldots, \mathrm{NP}_{h}(f)
$$

respectively. We call the set $\left\{\mathrm{NP}_{j}(f)\right\}_{j=1}^{h}$ the Newton polygons of $f$. 
Assume that we make the precisely the same substitutions $\left\{\Phi_{j}\right\}$ in some polynomial $H$.

Definition 3.6. Define

$$
H_{j}=H \circ \Phi_{1} \circ \cdots \circ \Phi_{j-1}
$$

for $j=2, \ldots, h$ and define the Newton polygons of $H$ as

$$
\mathrm{NP}_{1}(H)=\mathrm{NP}(H), \mathrm{NP}_{2}(H)=\mathrm{NP}\left(H_{2}\right), \ldots, \mathrm{NP}_{h}(H)=\mathrm{NP}\left(H_{h}\right) .
$$

In Lemma 3.10 we establish that $\mathrm{NP}_{k}(f)$ has just one key line segment as given in the following diagram:

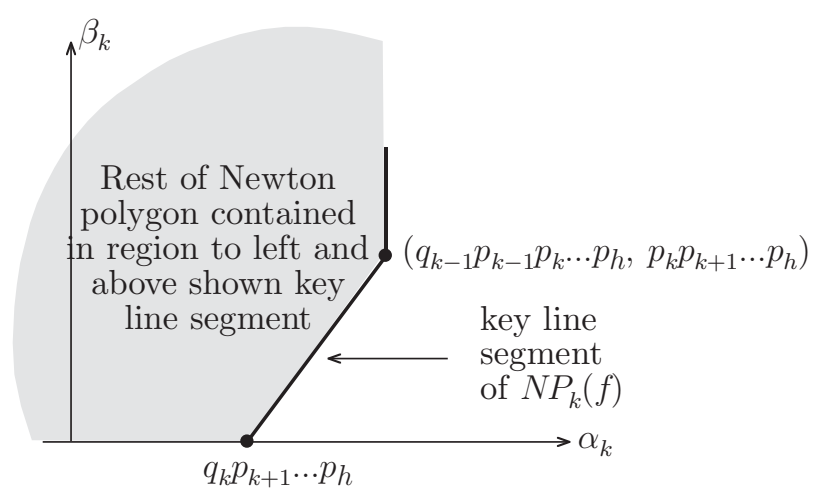

This key line segment shown on the diagram is the one used to find the Newton pair $\left(p_{k}, q_{k}\right)$ in the splice diagram. The line segment has slope $\tau_{k}$ and we know that we must obtain just one Newton pair from this line segment. Thus the terms in $f\left(x_{k}, y_{k}\right)$ corresponding to this line segment must be of the form

$$
c_{k} x_{k}^{q_{k} p_{k} \cdots p_{h}}\left(y_{k}^{p_{k}}-t_{k}^{p_{k}} x_{k}^{-\tau_{k}}\right)^{p_{k+1} \cdots p_{h}}
$$

and every integer point on the key line segment must be a key point of the Newton polygon.

In section 3.5 we use induction to establish the following results about the Newton polygons of the components of $f, g_{k}^{p_{k} \cdots p_{h}}$ and $a_{\gamma_{0} \ldots \gamma_{k}} g_{0}^{\gamma_{0}} \cdots g_{k}^{\gamma_{k}}$.

(i) Neither

$$
\mathrm{NP}_{k}\left(g_{k}^{p_{k} \cdots p_{h}}\right)
$$

nor any

$$
\mathrm{NP}_{k}\left(a_{\gamma_{0} \ldots \gamma_{k}} g_{0}^{\gamma_{0}} g_{1}^{\gamma_{1}} \cdots g_{k}^{\gamma_{k}}\right)
$$

where $a_{\gamma_{0} \ldots \gamma_{k}} g_{0}^{\gamma_{0}} g_{1}^{\gamma_{1}} \cdots g_{k}^{\gamma_{k}} \neq 0$ has a key line segment parallel to that of $\mathrm{NP}_{k}(f)$ (Lemmas 3.15 and 3.24). 
(ii) The corner point of $\operatorname{NP}_{k}\left(a_{\gamma_{0} \ldots \gamma_{k}} g_{0}^{\gamma_{0}} \cdots g_{k}^{\gamma_{k}}\right)$ is at

$$
\begin{aligned}
\alpha_{k} & =p_{1} \cdots p_{k-1} \gamma_{0}+q_{1} p_{2} \cdots p_{k-1} \gamma_{1}+\cdots+q_{k-1} \gamma_{k-1}+q_{k-1} p_{k-1} \gamma_{k} . \\
\beta_{k} & =\gamma_{k}
\end{aligned}
$$

(Corollary 3.20 to Lemma 3.19).

(iii) The Newton polygons, $\mathrm{NP}_{k}\left(g_{k}^{p_{k} \cdots p_{h}}\right)$ and $\mathrm{NP}_{k}\left(a_{\gamma_{0} \ldots \gamma_{k}} g_{0}^{\gamma_{0}} \cdots g_{k}^{\gamma_{k}}\right)$, have no point lying to the right of $\mathrm{NP}(f)$ and thus their corner points do not lie to the right of $\mathrm{NP}_{k}(f)$ (Lemmas 3.15 and 3.25). No two of these corner points coincide (Lemma 3.23).

(iv) For each key point on the key line segment of $\mathrm{NP}_{k}(f)$, that is, $\left(\alpha_{k}, \beta_{k}\right)$ where

$$
\begin{aligned}
\alpha_{k} & =q_{k-1} p_{k-1} p_{k} \cdots p_{h}-s \tau_{k} \\
\beta_{k} & =p_{k} \cdots p_{h}-s p_{k}
\end{aligned}
$$

for $s=1, \ldots,\left(p_{k} \cdots p_{h}\right)$, there exists a term in $\zeta_{k}$,

$$
a_{\gamma_{0} \ldots \gamma_{k}} g_{0}^{\gamma_{0}} \cdots g_{k}^{\gamma_{k}}
$$

such that the corner point of $\operatorname{NP}_{k}\left(a_{\gamma_{0} \ldots \gamma_{k}} g_{0}^{\gamma_{0}} \cdots g_{k}^{\gamma_{k}}\right)$ is this key point (Lemma 3.26).

(v) For $i=1, \ldots, k, \mathrm{NP}_{i}\left(g_{k+1}\right)$ has the same key line segment as $\mathrm{NP}_{i}(f)$ and the terms in $g_{k+1, i}$ and $f_{i}$ corresponding to this key line segment are the same. This implies that the first $k$ Newton pairs, and hence cabling pairs, of $f_{i}$ and $g_{k+1, i}$ are identical (Lemmas 3.17 and 3.29).

Diagrammatically the above can be illustrated as follows:

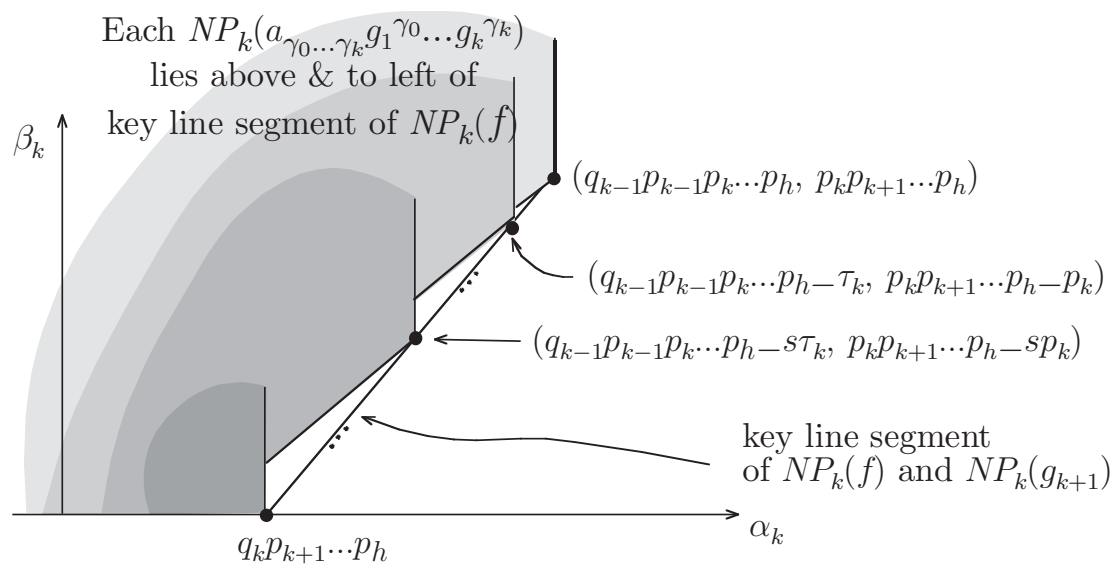




\section{Step 3: Proof of Theorem 1.1}

Proof of Theorem 1.1. The result is clear for $k=1$ as

$$
\psi_{1}=q_{1} p_{1} \cdots p_{h}, \quad q_{1}>1 \quad \text { and } \quad \delta_{0}=p_{1} \cdots p_{h}
$$

so that

$$
\psi_{1} \in \text { semi-group }\left\{\delta_{0}\right\} .
$$

Now consider $q_{k}$. As described in step 2, result (ii), there is some term in $f$,

$$
a_{\gamma_{0}^{\prime} \ldots \gamma_{k}^{\prime}} g_{0}^{\gamma_{0}^{\prime}} \cdots g_{k}^{\gamma_{k}^{\prime}}
$$

say, with $a_{\gamma_{0}^{\prime} \ldots \gamma_{k}^{\prime}} \neq 0$ whose Newton polygon $\mathrm{NP}_{k}\left(a_{\gamma_{0}^{\prime} \ldots \gamma_{k}^{\prime}} g_{0}^{\gamma_{0}^{\prime}} g_{1}^{\gamma_{1}^{\prime}} \cdots g_{k}^{\gamma_{k}^{\prime}}\right)$ has corner point at the key point, $\left(q_{k-1} p_{k-1} p_{k} \cdots p_{h}-\tau_{k}, p_{k} \cdots p_{h}-p_{k}\right)$ on $\mathrm{NP}_{k}(f)$. Thus

$$
\gamma_{k}^{\prime}=p_{k} \cdots p_{h}-p_{k}
$$

and

$$
\begin{aligned}
p_{1} \cdots p_{k-1} \gamma_{0}^{\prime}+q_{1} p_{2} \cdots p_{k-1} \gamma_{1}^{\prime}+\cdots+q_{k-1} \gamma_{k-1}^{\prime}+q_{k-1} p_{k-1} \gamma_{k}^{\prime} \\
=q_{k-1} p_{k-1} p_{k} \cdots p_{h}-\tau_{k} .
\end{aligned}
$$

Therefore

$$
\begin{array}{r}
p_{1} \cdots p_{k-1} \gamma_{0}^{\prime}+q_{1} p_{2} \cdots p_{k-1} \gamma_{1}^{\prime}+\cdots+q_{k-1} \gamma_{k-1}^{\prime}+q_{k-1} p_{k-1}\left(p_{k} \cdots p_{h}-p_{k}\right) \\
=q_{k-1} p_{k-1} p_{k} \cdots p_{h}-\tau_{k}
\end{array}
$$

and, as $q_{k}=q_{k-1} p_{k-1} p_{k}-\tau_{k}($ from section 2.2$)$,

$$
p_{1} \cdots p_{k-1} \gamma_{0}^{\prime}+q_{1} p_{2} \cdots p_{k-1} \gamma_{1}^{\prime}+\cdots+q_{k-1} \gamma_{k-1}^{\prime}=q_{k-1} p_{k-1} p_{k}-\tau_{k}=q_{k} .
$$

Multiply each side by $p_{k} \cdots p_{h}$ to obtain

$$
\delta_{0} \gamma_{0}^{\prime}+\delta_{1} \gamma_{1}^{\prime}+\cdots+\delta_{k-1} \gamma_{k-1}^{\prime}=q_{k} p_{k} \cdots p_{h}=\psi_{k}
$$

As $\gamma_{i}^{\prime} \geq 0$, for all $i$, and from Lemma 3.10, $q_{k} \neq 0$, the result follows and

$$
\psi_{k} \in \text { semi-group }\left\{\delta_{0}, \ldots, \delta_{k-1}\right\}
$$

It remains to prove the details summarised above in steps 1 and 2 . 


\subsection{Approximate roots of polynomials}

We first need to understand the ideas behind the generalized Tschirnhausen transformation developed by Abhyankar and Moh ([3]). Let $f \in \mathbb{C}[y]$ be a monic polynomial of degree $n$ :

$$
f(y)=y^{n}+a_{n-1} y^{n-1}+a_{n-2} y^{n-2}+\cdots+a_{0} .
$$

One can use a substitution, replacing $y+\frac{a_{n-1}}{n}$ by $y$, to "kill off" the coefficient of $y^{n-1}$. This affine automorphism is a Tschirnhausen transformation ([29]). A useful way of looking at this is the following. Divide $f$ by $\left(y+\frac{a_{n-1}}{n}\right)^{n}$ and express $f$ in the form

$$
f(y)=\left(y+\frac{a_{n-1}}{n}\right)^{n}+r_{1}(y)
$$

where $c_{i} \in \mathbb{C}$ and $\operatorname{deg}_{y} \psi \leq n-2$. By dividing $r_{1}(y)$ by $\left(y+\frac{a_{n-1}}{n}\right)^{n-2}$ we obtain

$$
r_{1}(y)=c_{n-2}\left(y+\frac{a_{n-1}}{n}\right)^{n-2}+r_{2}(y)
$$

with $\operatorname{deg}_{y} r_{2} \leq n-3$. We can continue in this fashion until we find

$$
f(y)=\left(y+\frac{a_{n-1}}{n}\right)^{n}+\sum_{j=0}^{n-2} c_{j}\left(y+\frac{a_{n-1}}{n}\right)^{j}
$$

for some $c_{j} \in \mathbb{C}$.

Abhyankar and Moh generalized this as follows to find the $l^{\text {th }}$ approximate root of $f$ :

Lemma 3.7. Let $f \in \mathbb{C}[x, y]$ be monic in $y$ with $\operatorname{deg}_{y}(f)=k l, k, l \in \mathbb{Z}, k, l>1$. Then there exists a unique $g \in \mathbb{C}[x, y]$ such that $g$ is monic in $y, \operatorname{deg}_{y}(g)=k$ and

$$
f(x, y)=g^{l}+\zeta
$$

where $\operatorname{deg}_{y}(\zeta)<(l-1) k=(l-1) \operatorname{deg}_{y}(g)$.

The proof of Lemma 3.7 is in [3]. We will use this lemma to show the following:

\section{Lemma 3.8. Let}

- $f \in \mathbb{C}[x, y]$ be monic in $y$ with $\operatorname{deg}_{y}(f)=k l m, k, l, m \in \mathbb{Z}, k, l, m>1$,

- $h$ be the $m^{\text {th }}$ approximate root of $f$ and

- $g$ be the $l^{\text {th }}$ approximate root of $h$.

Then $g$ is the $l m^{\text {th }}$ approximate root of $f$. 
Proof. As $h$ is the $m^{\text {th }}$ approximate root of $f$,

$$
f=h^{m}+\zeta
$$

where $\operatorname{deg}_{y}(\zeta)<(m-1) k l=(m-1) \operatorname{deg}_{y}(h)$. As $g$ is the $l^{\text {th }}$ approximate root of $h$,

$$
h=g^{l}+\phi
$$

where $\operatorname{deg}_{y}(\phi)<(l-1) k=(l-1) \operatorname{deg}_{y}(g)$. Thus

$$
\begin{aligned}
f & =h^{m}+\zeta \\
& =\left(g^{l}+\phi\right)^{m}+\zeta \\
& =g^{l m}+\sum_{i=1}^{m}\left(\begin{array}{c}
m \\
i
\end{array}\right) \phi^{i} g^{l(m-i)}+\zeta .
\end{aligned}
$$

Hence

$\operatorname{deg}_{y}\left(f-g^{l m}\right) \leq \operatorname{deg}_{y}(\phi)+(m-1) \operatorname{deg}_{y}\left(g^{l}\right)<k l-k+(m-1) k l=(m l-1) \operatorname{deg}_{y}(g)$.

By the uniqueness of approximate roots, $g$ is the $l m^{\text {th }}$ approximate root of $f$.

The main result we need for the proof of Theorem 1.1 is as follows:

Lemma 3.9. Let $f \in \mathbb{C}[x, y]$ be monic in $y$ and $\operatorname{deg}_{y}(f)=p_{1} p_{2} \cdots p_{k} \cdots p_{h}$. Let $g_{0}=x$ and $g_{1}, g_{2}, \ldots, g_{k}$ be the $\left(p_{1} p_{2} \cdots p_{h}\right)^{t h},\left(p_{2} \cdots p_{h}\right)^{t h}, \ldots,\left(p_{k} \cdots p_{h}\right)^{\text {th }}$ approximate roots of $f$ respectively. Then we can express $f$ as a sum of polynomials as follows. Given

$$
f=g_{k}^{p_{k} p_{k+1} \cdots p_{h}}+\zeta_{k}
$$

then

$$
\zeta_{k}=\sum_{\gamma_{0}, \ldots, \gamma_{k}} a_{\gamma_{0} \ldots \gamma_{k}} g_{0}^{\gamma_{0}} g_{1}^{\gamma_{1}} \cdots g_{k}^{\gamma_{k}}
$$

with $0 \leq \gamma_{0}, 0 \leq \gamma_{1}<p_{1}, \ldots, 0 \leq \gamma_{k-1}<p_{k-1}, 0 \leq \gamma_{k}<p_{k} p_{k+1} \cdots p_{h}-1$.

The proof is given by Suzuki in [28].

\subsection{The Newton polygons of $f$}

We have assumed that the cabling pairs of $f$ are either just $\{(1,0)\}$ or, for some $h \geq 1$,

$$
\left\{\left(p_{1}, q_{1}\right),\left(p_{2}, q_{2}\right), \ldots,\left(p_{h}, q_{h}\right)\right\}
$$

with $p_{i}>1$ for all $i$. (The case where there are redundant cabling pairs of the form $(1, r)$ is addressed in section 3.6.)

The attributes of $\mathrm{NP}_{1}(f)$ are as follows. If $f \in \mathbb{C}[x, y]$ is irreducible and $f(x, y)=0$ defines a curve in $\mathbb{C}^{2}$ with one place at infinity, its Newton polygon, assuming that $f$ has a non-zero constant term, is one of the following: 
1.

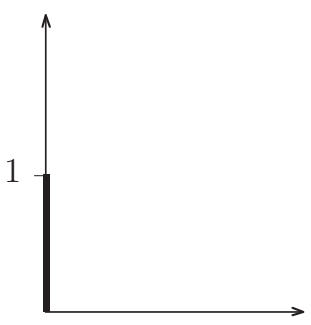

2.

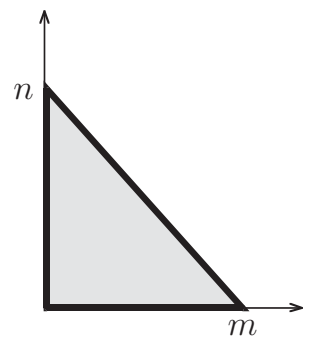

In case $1, f(x, y)=y$, the curve is just a line and has splice diagram

Thus for case 1 , Theorem 1.1 is trivially true. Case 2 is the interesting case and we discuss this in detail. As there is only one place at infinity we know that the key line segment of $\mathrm{NP}_{1}(f)$ will give exactly one Newton pair. As we have assumed that the curve meets infinity at $y=0, n>m$. From the splice diagram, we see that the root node has linking number $\delta_{0}=p_{1} p_{2} \cdots p_{h}$. So the degree of the polynomial is also $n=p_{1} p_{2} \cdots p_{h}$ (see section 2 ). Thus we can assume, without loss of generality and by applying, where necessary, polynomial automorphisms of $\mathbb{C}^{2}$ as described in Neumann and Wightwick ([20]) and Wightwick ([32]) that

- $n>m$ so that $n=p_{1} p_{2} \cdots p_{h}$.

- $m>1$.

- $f$ is monic in $y$ and

$$
f(x, y)=y^{n}+\sum_{j=2}^{n} a_{j} y^{n-j}
$$

where $a_{j} \in \mathbb{C}[x]$.

- $f$ is monic in $x$. As the line segment in the boundary of the Newton polygon has slope $-p_{1} / q_{1}, m=\operatorname{deg}_{x}(f)=q_{1} p_{2} p_{3} \cdots p_{h}$.

- $q_{1} \neq 1$ and $p_{2}, \ldots, p_{h}>1$. If any of $p_{2}, p_{3}, \ldots, p_{h}=1$, the splice diagram would not be minimal. 
Given the above degrees, the polynomial, $f$, is of the form

$$
f(x, y)=\sum_{p_{1} \alpha+q_{1} \beta=q_{1} p_{1} p_{2} \cdots p_{h}} a_{\alpha \beta} x^{\alpha} y^{\beta}+\sum_{p_{1} \alpha+q_{1} \beta<q_{1} p_{1} p_{2} \cdots p_{h}} a_{\alpha \beta} x^{\alpha} y^{\beta}
$$

or, alternatively,

$$
f(x, y)=\left(y^{p_{1}}-t_{1}^{p_{1}} x^{q_{1}}\right)^{p_{2} p_{3} \cdots p_{h}}+\sum_{p_{1} \alpha+q_{1} \beta<q_{1} p_{1} p_{2} \cdots p_{h}} a_{\alpha \beta} x^{\alpha} y^{\beta}
$$

with $t_{1}^{p_{1}}=1$.

Lemma 3.10. At the $k^{\text {th }}$ stage, $k=2,3, \ldots, h$, of finding Newton pairs, the Newton polygon, $\mathrm{NP}_{k}(f)$,

(i) has one key line segment with end points

$$
\left(q_{k} p_{k+1} \cdots p_{h}, 0\right) \quad \text { and }\left(q_{k-1} p_{k-1} p_{k} p_{k+1} \cdots p_{h}, p_{k} p_{k+1} \cdots p_{h}\right),
$$

(ii) has key points at

$$
\alpha_{k}=q_{k} p_{k} \cdots p_{h}-s \tau_{k} \quad \text { and } \quad \beta_{k}=p_{k} \cdots p_{h}-s p_{k}
$$

for $s=0, \ldots, p_{k+1} \cdots p_{h}$ and

(iii) has right side boundary components of the following form:

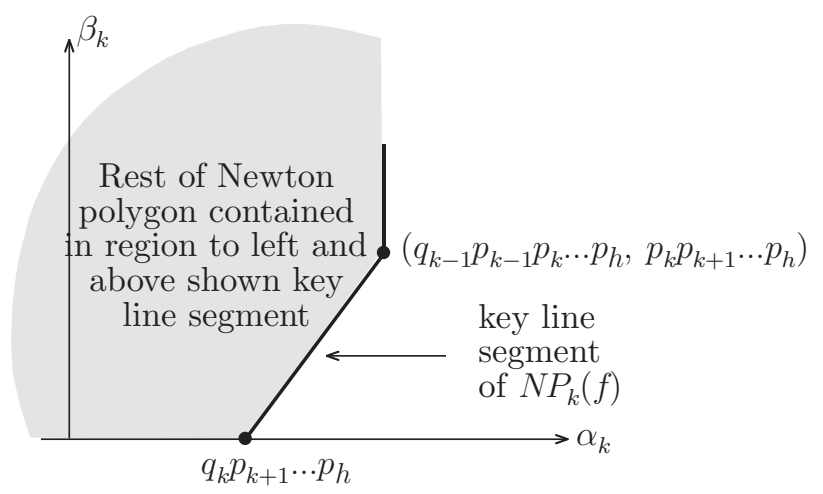

(iv) is such that $q_{k} \neq 0$.

Proof. We use induction for (i), (ii), and (iii). From equation 3.3 we have a quasihomogeneous polynomial which corresponds to the key line segment in $\mathrm{NP}_{1}(f)$ being

$$
\left(y^{p_{1}}-t_{1}^{p_{1}} x^{q_{1}}\right)^{p_{2} p_{3} \cdots p_{h}}
$$


Our first substitution is

$$
x=x_{2}^{p_{1}}, \quad y=x_{2}^{q_{1}}\left(t_{1}+y_{2}\right) .
$$

The quasi-homogeneous polynomial becomes

$$
x_{2}^{q_{1} p_{1}}\left(\left(t_{1}+y_{2}\right)^{p_{1}}-t_{1}^{p_{1}}\right)^{p_{2} p_{3} \cdots p_{h}} .
$$

The powers of the terms in this lie on the vertical line segment $\alpha_{2}=q_{1} p_{1} p_{2} \cdots p_{h}$, $p_{2} \cdots p_{h} \leq \beta_{2} \leq p_{1} p_{2} \cdots p_{h}$ which forms part of the right boundary of $\mathrm{NP}_{2}(f)$. All other key points satisfy $\alpha_{2}<q_{1} p_{1} p_{2} \cdots p_{h}$.

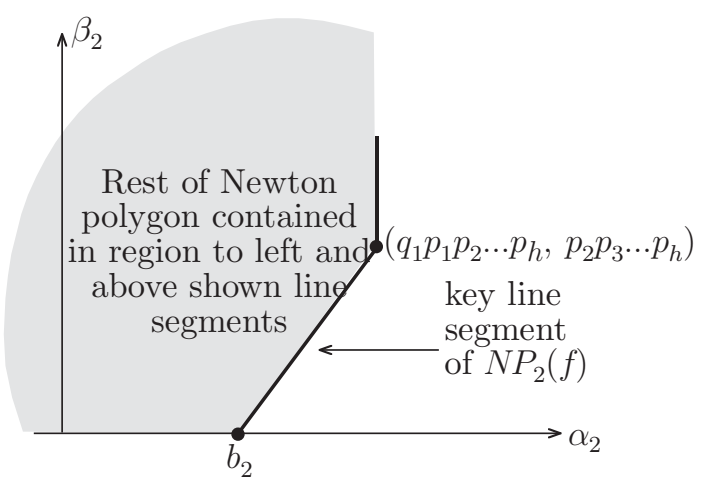

As the curve, $C$, has just one place at infinity, there is just one key line segment in $\mathrm{NP}_{2}(f)$ and this has slope $p_{2} / \tau_{2}$ where $\tau_{2}=p_{1} q_{1} p_{2}-q_{2}$. The corresponding terms in $f_{2}$ are

$$
c_{2} x_{2}^{q_{1} p_{1} \cdots p_{k}}\left(y_{2}^{p_{2}}-t_{2}^{p_{2}} x_{2}^{-\tau_{2}}\right)^{p_{3} \cdots p_{h}}
$$

for some $c_{2}, t_{2} \in \mathbb{C}$, and the result follows.

Now assume that at stage $k-1, \mathrm{NP}_{k-1}(f)$ has a key line segment as follows:

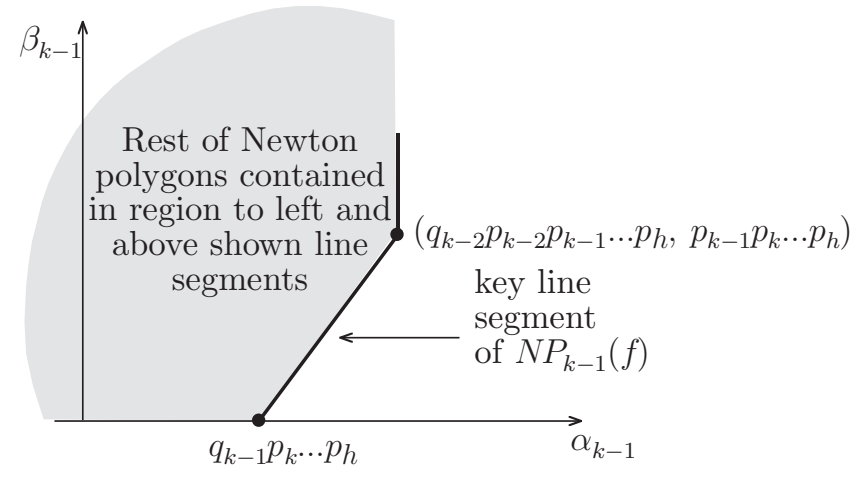


and this key line segment corresponds to a quasi-homogeneous polynomial

$$
c_{k-1} x_{k-1}^{q_{k-2} p_{k-2} \cdots p_{h}}\left(y_{k-1}^{p_{k-1}}-t_{2}^{p_{k-1}} x_{k-1}^{-\tau_{k-1}}\right)^{p_{k} \cdots p_{h}} .
$$

Substituting

$$
x_{k-1}=x_{k}^{p_{k-1}}, \quad y_{k-1}=x_{k}^{-\tau_{k-1}}\left(t_{k-1}+y_{k}\right),
$$

this quasi-homogeneous polynomial becomes

$$
\begin{aligned}
\left(c_{k-1} x_{k}^{\left.\left(q_{k-2} p_{k-2} \cdots p_{h}\right) p_{k-1}-\tau_{k-1} p_{k-1} \cdots p_{h}\right)}\left(\left(t_{k-1}+y_{k}\right)^{p_{k-1}}-t_{k-1}^{p_{k-1}}\right)^{p_{k} p_{k+1} \cdots p_{h}}\right. & \\
= & c_{k-1} x_{k}^{q_{k-1} p_{k-1} \cdots p_{h}}\left(\left(t_{k-1}+y_{k}\right)^{p_{k-1}}-t_{k-1}^{p_{k-1}}\right)^{p_{k} p_{k+1} \cdots p_{h}}
\end{aligned}
$$

As the quasi-homogeneous polynomial maps to terms in $f_{k}$ which match the vertical line segment in the right boundary of $\mathrm{NP}_{k}(f)$, we obtain the corner point as shown:

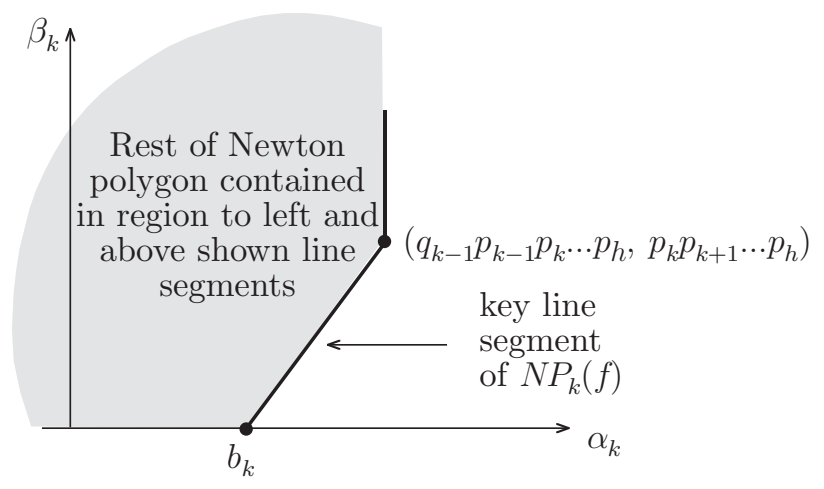

Finally, as the curve, $C$, has one place at infinity, we have one key line segment of slope $p_{k} / \tau_{k}$ where $\tau_{k}=q_{k-1} p_{k-1} p_{k}-q_{k}$ with corresponding equation

$$
c_{k} x_{k}^{q_{k} p_{k} \cdots p_{h}}\left(y_{k}^{p_{k}}-t_{k}^{p_{k}} x_{k}^{-\tau_{k}}\right)^{p_{k+1} \cdots p_{h}}
$$

which gives the required key points.

For (iv), if $q_{k}=0$, we obtain $\tau_{k}=q_{k-1} p_{k-1} p_{k}$. But then $\operatorname{gcd}\left(p_{k}, \tau_{k}\right) \neq 1$ which is a contradiction.

\subsection{Basic facts about Newton polygons}

Lemma 3.11. Let $f, g \in \mathbb{C}\left[x, x^{-1}, y\right], f, g \neq 0$. Let $\mathrm{NP}(f)$ denote the Newton polygon of $f$. Then

$$
\mathrm{NP}(f g)=\mathrm{NP}(f)+\mathrm{NP}(g)
$$

where the sum of two sets consists of all the sums of each element in the first set with each element in the second set. 
This well known fact is left as an exercise in many references and a proof is presented in Schinzel ([26, Theorem 18, page 89]).

Corollary 3.12. The Newton polygon of $f^{n}$ is similar to the Newton polygon of $f$.

We will need to have some understanding of how the corner point of $f g$ relates to the corner points of $f$ and $g$.

Corollary 3.13. If $f$ is such that $\operatorname{NP}(f)$ has a key line segment with corner point $(b, c)$ and point on the horizontal axis, $(a, 0)$, and $g$ is such that $\mathrm{NP}(g)$ has a corner point on the horizontal axis, $(d, 0)$ :
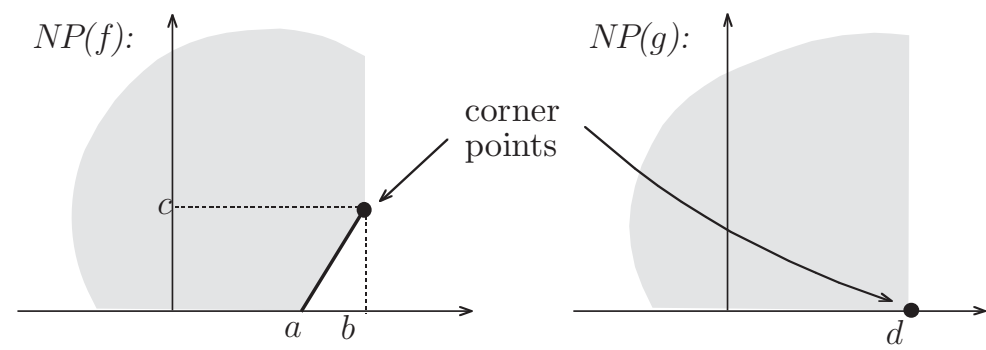

Rest of Newton polygons contained in region to left and above shown line segment and corner points.

then $\operatorname{NP}(f g)$ has a key line segment from $(a+d, 0)$ to $(b+d, c)$, with the latter point being the corner point:

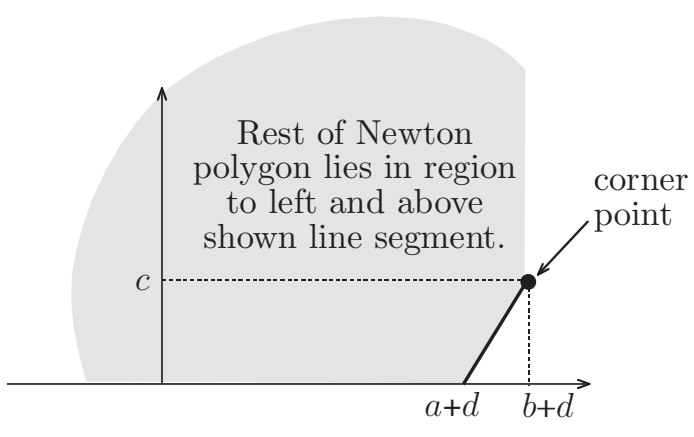


If $f$ is such that $\mathrm{NP}(f)$ has a corner point on the horizontal axis at $(d, 0)$, and $g$ is such that $\mathrm{NP}(g)$ has no key line segment and has a corner point at $(b, c)$ :
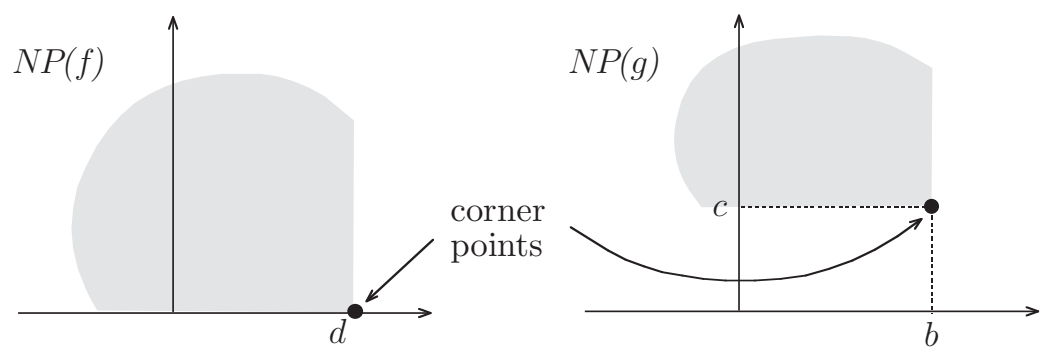

Rest of Newton polygons contained in regions to left and above shown corner points.

then $\operatorname{NP}(f g)$ has no key line segment and has a corner point at $(b+d, c)$ :

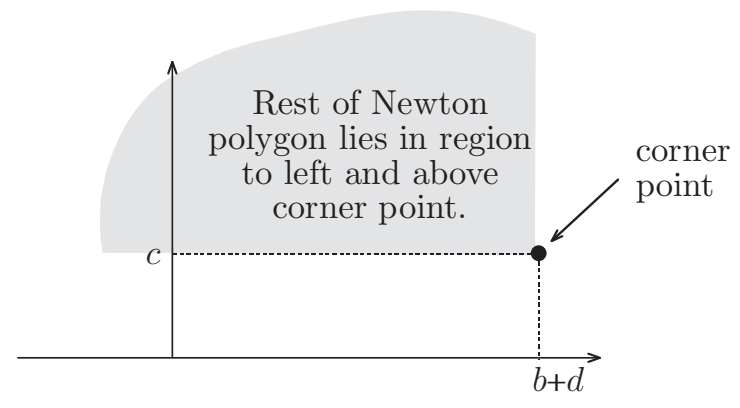

Lemma 3.14. Assume that we have a key line segment of non-zero slope in the boundary of $\mathrm{NP}(f)$ with key points $\left(\alpha_{1}, \beta_{1}\right)$ and $\left(\alpha_{2}, \beta_{2}\right)$ such that

- $\left(\alpha_{1}, \beta_{1}\right)$ is the end point of the line segment for which $\beta_{1}$ is maximum and

- $\left(\alpha_{2}, \beta_{2}\right)$ is the nearest key point to $\left(\alpha_{1}, \beta_{1}\right)$ on the line segment.

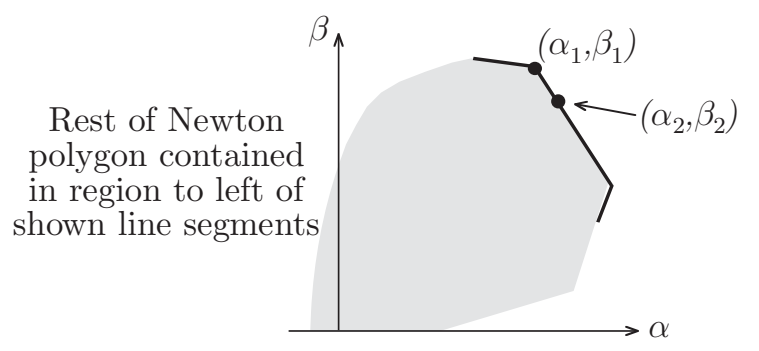


Then $\operatorname{NP}\left(f^{m}\right)$ will have key points in its boundary at

$$
\left(m \alpha_{1}, m \beta_{1}\right) \quad \text { and } \quad\left(m \alpha_{1}-\left(\alpha_{1}-\alpha_{2}\right), m \beta_{1}-\left(\beta_{1}-\beta_{2}\right)\right) .
$$

Proof. The terms in $f$ corresponding to the line segment will be of the form

$$
a_{1} x^{\alpha_{1}} y^{\beta_{1}}+a_{2} x^{\alpha_{2}} y^{\beta_{2}}+\sum_{\beta<\beta_{2}} a_{\beta} x^{\alpha} y^{\beta},
$$

$a_{1}, a_{2} \neq 0$. Taking the $m^{\text {th }}$ power of this gives

$$
\left(a_{1} x^{\alpha_{1}} y^{\beta_{1}}+a_{2} x^{\alpha_{2}} y^{\beta_{2}}\right)^{m}+r
$$

where

$$
r=\sum_{j=1}^{m}\left(\begin{array}{c}
m \\
j
\end{array}\right)\left(a_{1} x^{\alpha_{1}} y^{\beta_{1}}+a_{2} x^{\alpha_{2}} y^{\beta_{2}}\right)^{m-j}\left(\sum_{\beta<\beta_{2}} a_{\beta} x^{\alpha} y^{\beta}\right)^{j} .
$$

As $\operatorname{deg}_{y}(r)<\beta_{1}(m-j)+j \beta_{2}$ this has no term

$$
y^{\beta_{1} m} \quad \text { or } \quad y^{\beta_{1}(m-1)+\beta_{2}}
$$

with non-zero coefficient in $\mathbb{C}\left[x^{-1}, x, y\right]$. However

$$
\left(a_{1} x^{\alpha_{1}} y^{\beta_{1}}+a_{2} x^{\alpha_{2}} y^{\beta_{2}}\right)^{m}
$$

does have such a term with non-zero coefficient.

\subsection{Technical lemmas}

In this section we use induction to establish the technical lemmas used in the proof of Theorem 1.1.

Remark. We show that for $i=1, \ldots, k, \mathrm{NP}_{i}\left(g_{k+1}\right)$ has the same same key line segment as $\mathrm{NP}_{i}(f)$ and the terms in $g_{k+1, i}$ and $f_{i}$ corresponding to this key line segment are the same. This implies that the first $k$ Newton pairs, and hence cabling pairs, of $f_{i}$ and $g_{k+1, i}$ are identical (Lemmas 3.17 and 3.29). From Lemma 3.17 we see that $g_{k+1}$, $k=1, \ldots, h-1$, meets infinity at one point, $y=0$. In addition, $\operatorname{deg}_{y}\left(g_{k+1}\right)=$ $p_{1} p_{2} \cdots p_{k}$, while from above the first $k$ cabling pairs of $g_{k+1}$ are $\left\{\left(p_{1}, q_{1}\right), \ldots,\left(p_{k}, q_{k}\right)\right\}$. Thus the splice diagram for $g_{k+1}$ is

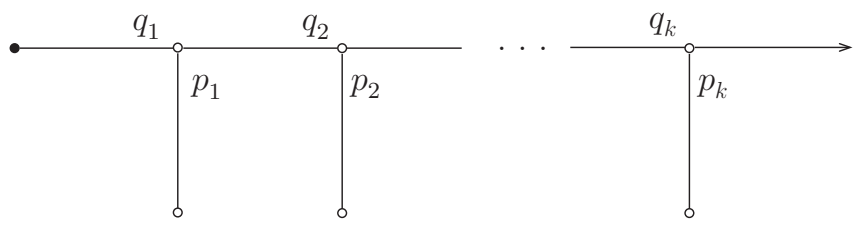


and the link at infinity of $g_{k+1}$ corresponds to the virtual component of the link at infinity of $f, S_{v_{k}}$.

We start by establishing what occurs for $\mathrm{NP}_{1}$. We use the fact that

$$
f=g_{1}^{p_{1} \cdots p_{h}}+\zeta_{1} \quad \text { and } \quad \zeta_{1}=\sum a_{\alpha \beta} g_{0}^{\alpha} g_{1}^{\beta}
$$

with $\beta<p_{1} \cdots p_{h}-1$.

Lemma 3.15. $\mathrm{NP}_{1}\left(g_{1}^{p_{1} \cdots p_{h}}\right)$ and $\mathrm{NP}_{1}\left(a_{\alpha \beta} g_{0}^{\alpha} g_{1}^{\beta}\right)$ have no key line segments parallel to that of $\mathrm{NP}_{1}(f)$ and their unique corner points lie within $\mathrm{NP}_{1}(f)$.

Proof. This is obvious as $g_{0}=x$ and $g_{1}=y$.

Lemma 3.16. $\mathrm{NP}_{1}\left(g_{k+1}^{p_{k+1} \cdots p_{h}}\right)$ is contained within $\mathrm{NP}_{1}(f)$ for $k=0, \ldots, h-1$ and includes the key point $\left(0, p_{1} \cdots p_{h}\right)$.

Proof. Recall that

$$
f=g_{k+1}^{p_{k+1} \cdots p_{h}}+\zeta_{k+1}
$$

with $\zeta_{k+1} \in \mathbb{C}[x, y], \operatorname{deg}_{y}\left(\zeta_{k+1}\right)<p_{1} \cdots p_{h}-p_{1} \cdots p_{k}$ and

$$
g_{k+1}=g_{1}^{p_{1} \cdots p_{k}}+\phi
$$

with $\operatorname{deg}_{y}(\phi)<p_{1} \cdots p_{k}-1$ (Lemma 3.8). Thus $\left(0, p_{1} \cdots p_{k}\right)$ is a key point on the boundary of $\mathrm{NP}_{1}\left(g_{k+1}\right)$. Assume we have a key point in $\mathrm{NP}_{1}\left(g_{k+1}\right),\left(\alpha^{\prime}, \beta^{\prime}\right)$ say, which satisfies

- $p_{1} \alpha^{\prime}+q_{1} \beta^{\prime}>q_{1} p_{1} \cdots p_{k}$,

- $\left(\alpha^{\prime}, \beta^{\prime}\right)$ lies on the boundary of $\mathrm{NP}_{1}\left(g_{k+1}\right)$,

- $\beta^{\prime}$ is the maximum possible such value.

Then $\left(0, p_{1} \cdots p_{k}\right)$ and $\left(\alpha^{\prime}, \beta^{\prime}\right)$ lie on the same line segment in the boundary of $\mathrm{NP}_{1}\left(g_{k+1}\right)$ and there are no key points between them. $\mathrm{NP}_{1}\left(g_{k+1}\right)$ is as follows:

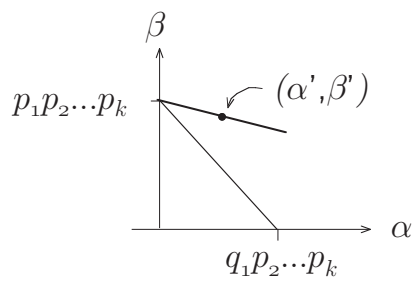


From Lemma 3.14 it follows that $\mathrm{NP}_{1}\left(g_{k+1}^{p_{k+1} \cdots p_{h}}\right)$ has key points in its boundary at

$$
\left(0, p_{1} \cdots p_{h}\right) \quad \text { and } \quad\left(\alpha^{\prime}, p_{1} \cdots p_{h}-\left(p_{1} \cdots p_{k}-\beta^{\prime}\right)\right) .
$$

Thus there is a term in $g_{k+1}^{p_{k+1} \cdots p_{h}}, x^{\alpha^{\prime}} y^{p_{1} \cdots p_{h}-\left(p_{1} \cdots p_{k}-\beta^{\prime}\right)}$ with non-zero coefficient. The key point lies outside $\mathrm{NP}_{1}(f)$ as

$$
\begin{aligned}
p_{1} \alpha^{\prime}+q_{1}\left(p_{1} \cdots p_{h}-\left(p_{1} \cdots p_{k}-\beta^{\prime}\right)\right) & =p_{1} \alpha^{\prime}+q_{1} \beta^{\prime}+q_{1} p_{1} \cdots p_{h}-q_{1} p_{1} \cdots p_{k} \\
& >q_{1} p_{1} \cdots p_{h}
\end{aligned}
$$

Thus there must be a term in $\zeta_{k+1}$ which cancels the term $x^{\alpha^{\prime}} y^{p_{1} \cdots p_{h}-\left(p_{1} \cdots p_{k}-\beta^{\prime}\right)}$. But $\operatorname{deg}_{y}\left(\zeta_{k+1}\right)<p_{1} \cdots p_{h}-p_{1} \cdots p_{k}$ so this is not possible. Thus $\operatorname{NP}_{1}\left(g_{k+1}^{p_{k+1} \cdots p_{h}}\right)$ is contained in $\mathrm{NP}_{1}(f)$.

Lemma 3.17. For $k=1, \ldots, h-1, \mathrm{NP}_{1}\left(g_{k+1}^{p_{k+1} \cdots p_{h}}\right)$ has the same key line segment as $\mathrm{NP}_{1}(f)$ and the terms in $g_{k+1}^{p_{k+1} \cdots p_{h}}$ and $f$ corresponding to these are identical.

Proof. As $\mathrm{NP}_{1}\left(g_{k+1}^{p_{k+1} \cdots p_{h}}\right)$ is contained in $\mathrm{NP}_{1}(f), \mathrm{NP}_{1}\left(g_{k+1}\right)$ is contained in the given region:

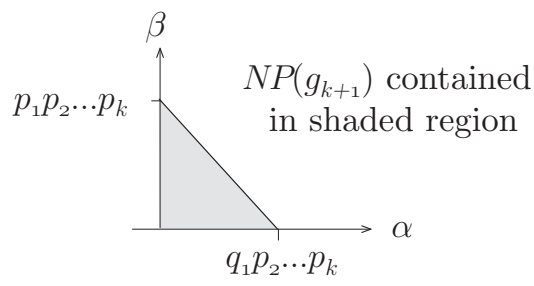

Thus

$$
g_{k+1}=\sum_{i=0}^{p_{2} \cdots p_{k}} d_{i} x^{q_{1} i} y^{p_{1}\left(p_{2} \cdots p_{k}-i\right)}+\sum_{p_{1} \alpha+q_{1} \beta<q_{1} p_{1} \cdots p_{k}} d_{\alpha \beta} x^{\alpha} y^{\beta}
$$

for some $d_{i}, d_{\alpha \beta} \in \mathbb{C}$. Note that $d_{0}=1$. Take the $\left(p_{k+1} \cdots p_{h}\right)^{\text {th }}$ power of this to obtain

$$
g_{k+1}^{p_{k+1} \cdots p_{h}}=\left(\sum_{i=0}^{p_{1} \cdots p_{k}} d_{i} x^{q_{1} i} y^{p_{1}\left(p_{2} \cdots p_{k}-i\right)}\right)^{p_{k+1} \cdots p_{h}}+\sum_{p_{1} \alpha+q_{1} \beta<q_{1} p_{1} \cdots p_{k}} d_{\alpha \beta}^{\prime} x^{\alpha} y^{\beta} .
$$

Equate $g_{k+1}^{p_{k+1} \cdots p_{h}}+\zeta_{k+1}$ to

$$
\begin{aligned}
f & =\left(y^{p_{1}}-t_{1}^{p_{1}} x^{q_{1}}\right)^{\left(p_{2} \cdots p_{k}\right)\left(p_{k+1} \cdots p_{h}\right)}+\sum_{p_{1} \alpha+q_{1} \beta<q_{1} p_{1} \cdots p_{k}} a_{\alpha \beta}^{\prime} x^{\alpha} y^{\beta} \\
& =\left(\sum_{j=0}^{p_{2} \cdots p_{k}}\left(\begin{array}{c}
p_{2} \cdots p_{k} \\
j
\end{array}\right)\left(y^{p_{1}}\right)^{\left(p_{2} \cdots p_{k}-j\right)}\left(t_{1}^{p_{1}} x^{q_{1}}\right)^{j}\right)^{p_{k+1} \cdots p_{h}}+\sum_{p_{1} \alpha+q_{1} \beta<q_{1} p_{1} \cdots p_{k}} a_{\alpha \beta}^{\prime} x^{\alpha} y^{\beta}
\end{aligned}
$$


As $\operatorname{deg}_{y}\left(\zeta_{k+1}\right)<p_{1} \cdots p_{h}-p_{1} \cdots p_{k}$, the terms with $y$-degree greater than $p_{1} \cdots p_{h}-$ $p_{1} \cdots p_{k}$ in $g_{k+1}^{p_{k+1} \cdots p_{h}}$ and $f$ must be equal. Hence

$$
\begin{aligned}
& d_{0} y^{p_{1} \cdots p_{h}}+\left(p_{k+1} \cdots p_{h}\right) y^{p_{1} \cdots p_{k}\left(p_{k+1} \cdots p_{h}-1\right)} \sum_{i=1}^{p_{2} \cdots p_{k}} d_{i}\left(x^{q_{1}}\right)^{i}\left(y^{p_{1}}\right)^{\left(p_{2} \cdots p_{k}-i\right)} \\
= & y^{p_{1} \cdots p_{h}}+\left(p_{k+1} \cdots p_{h}\right) y^{p_{1} \cdots p_{k}\left(p_{k+1} \cdots p_{h}-1\right)} \sum_{j=1}^{p_{2} \cdots p_{k}}\left(\begin{array}{c}
p_{k+1} \cdots p_{h} \\
j
\end{array}\right)\left(t_{1}^{p_{1}} x^{q_{1}}\right)^{j} y^{p_{1}\left(p_{2} \cdots p_{h}-j\right)}
\end{aligned}
$$

This uniquely determines the $d_{i}$ in terms of $t_{1}^{p_{1}}$ and we get the required values for each $d_{i}$.

Before looking at the more general case we need the following result.

Lemma 3.18. Let $f \in \mathbb{C}[x, y]$ have one place at infinity and $g_{j} \in \mathbb{C}\left[x^{-1}, x, y\right]$. Assume that the key line segment of $\mathrm{NP}_{j}(f)$ is as follows:

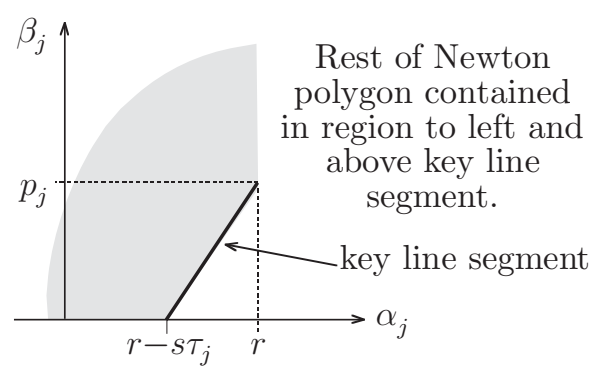

Assume that $\mathrm{NP}_{j}(g)=\mathrm{NP}\left(g_{j}\right)$ has no key line segment that is parallel to that of $\mathrm{NP}_{j}(f)$. Let $\phi:\left(x_{j}, y_{j}\right) \mapsto\left(x_{j+1}^{p_{j}}, x_{j+1}^{-\tau_{j}}\left(y+t_{j}\right)\right.$ be the substitution to be used to find the next Newton pair of $f$.

Then $\mathrm{NP}\left(g_{j} \circ \phi\right)=\mathrm{NP}\left(g_{j+1}\right)$ has a corner point on the horizontal axis with all other points in the Newton polygon lying above and to the left of that point.

Proof. The key line segment of $\mathrm{NP}_{j}(f)$ matches the following terms in $f_{j}$

$$
a x_{j}^{r}\left(y_{j}^{p_{j}}-t_{j}^{p_{j}} x_{j}^{-\tau_{j}}\right)^{s}
$$

for some constant $a$. Then $g_{j}$ can be expressed as

$$
g_{j}=c x_{j}^{\alpha^{\prime}} y_{j}^{\beta^{\prime}}+\sum_{p_{j} \alpha-\tau_{j} \beta<p_{j} \alpha^{\prime}-\tau_{j} \beta^{\prime}} d_{\alpha \beta} x_{j}^{\alpha} y_{j}^{\beta}
$$

for some $c, d_{\alpha \beta} \in \mathbb{C}$. Put

$$
\left(x_{j}, y_{j}\right)=\left(x_{j+1}^{p_{j}}, x_{j+1}^{-\tau_{j}}\left(y_{j+1}+t_{j}\right)\right)
$$


to obtain

$$
g_{j+1}=c x_{j+1}^{\alpha^{\prime} p_{j}-\beta^{\prime} \tau_{j}}\left(y_{j+1}+t_{j}\right)^{\beta^{\prime}}+\sum_{p_{j} \alpha-\tau_{j} \beta<p_{j} \alpha^{\prime}-\tau_{j} \beta^{\prime}} d_{\alpha \beta} x_{j+1}^{\alpha p_{j}-\beta \tau_{j}}\left(y_{j+1}+t_{j}\right)^{\beta} .
$$

One of the terms in $x_{j+1}^{\alpha^{\prime} p_{j}-\beta^{\prime} \tau_{j}}\left(y_{j+1}+t_{j}\right)^{\beta^{\prime}}$ gives a point lying in the right boundary of $\mathrm{NP}_{j+1}(g)$ at $\left(\alpha^{\prime} p_{j}-\beta^{\prime} \tau_{j}, 0\right)$. This point lies on the $\alpha_{j+1}$-axis as follows:

$N P\left(g_{j}\right):$

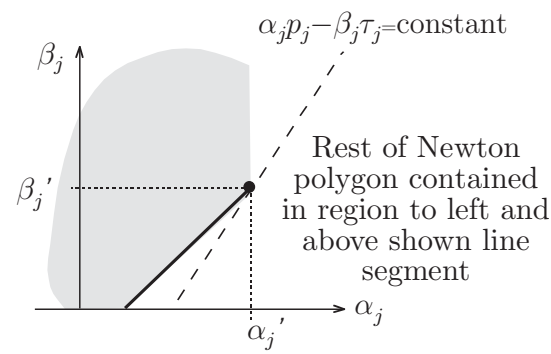

$N P\left(g_{j+1}\right):$

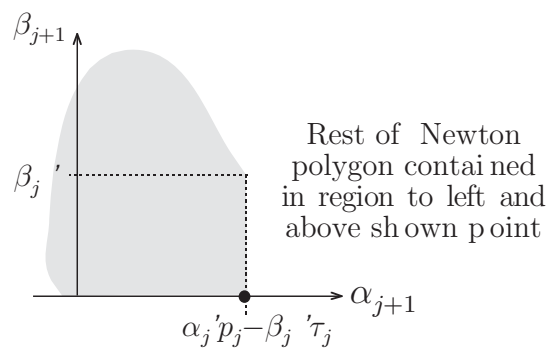

Now assume that we have established the following:

(i) that $\mathrm{NP}_{k-1}\left(g_{0}\right)=\mathrm{NP}_{k-1}(x)$ is a point on the $\alpha_{k-1}$-axis, $\left(p_{1} p_{2} \cdots p_{k-2}, 0\right)$.

(ii) that for $j=1,2, \ldots, k-2, \mathrm{NP}_{k-1}\left(g_{j}\right)$ has corner point, $\left(q_{j} p_{j+1} \cdots p_{k-2}, 0\right)$ with all other points in the Newton polygon lying above or to the left of that point.
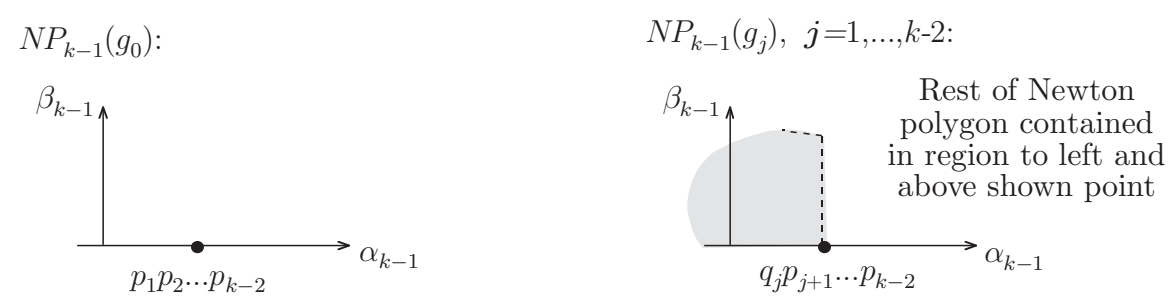

(iii) that $\mathrm{NP}_{k-1}\left(g_{k-1}\right)$ has a key line segment which is not parallel to the key line segment of $\mathrm{NP}_{k-1}(f)$ and has corner point $\left(p_{k-1} q_{k-2}, 1\right)$.

(iv) that $\mathrm{NP}_{k-1}\left(g_{k}^{p_{k} \cdots p_{h}}\right)$ and $\mathrm{NP}_{k-1}(f)$ have the same key line segments, and that the terms in $f$ which give this key line segment are also terms of $g_{k}^{p_{k} \cdots p_{h}}$. This means that $\mathrm{NP}_{k-1}\left(g_{k}\right)$ has a key line segment from $\left(q_{k-1}, 0\right)$ to $\left(p_{k-2} q_{k-2} p_{k-1}, p_{k-1}\right)$. 
$N P_{k-1}\left(g_{k-1}\right):$

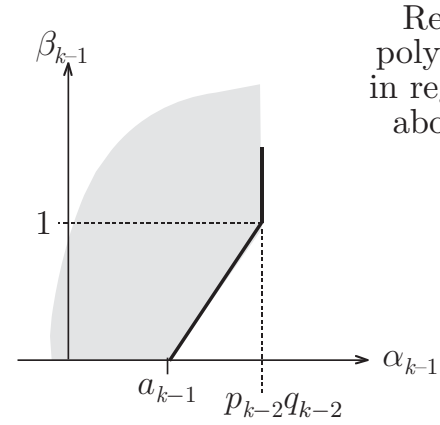

$N P_{k-1}\left(g_{k}\right):$

Rest of Newton polygons contained in region to left and above shown line segments

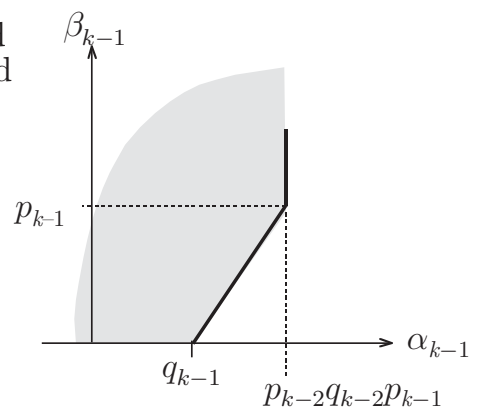

\section{Lemma 3.19. Then}

(i) $\mathrm{NP}_{k}\left(g_{0}\right)=\mathrm{NP}_{k}(x)$ is a point on the $\alpha_{k}$-axis, $\left(p_{1} p_{2} \cdots p_{k-1}, 0\right)$.

(ii) for $j=1,2, \ldots, k-2, \mathrm{NP}_{k}\left(g_{j}\right)$ has corner point, $\left(q_{j} p_{j+1} \cdots p_{k-1}, 0\right)$ with all other points in the Newton polygon lying above or to the left of that point.
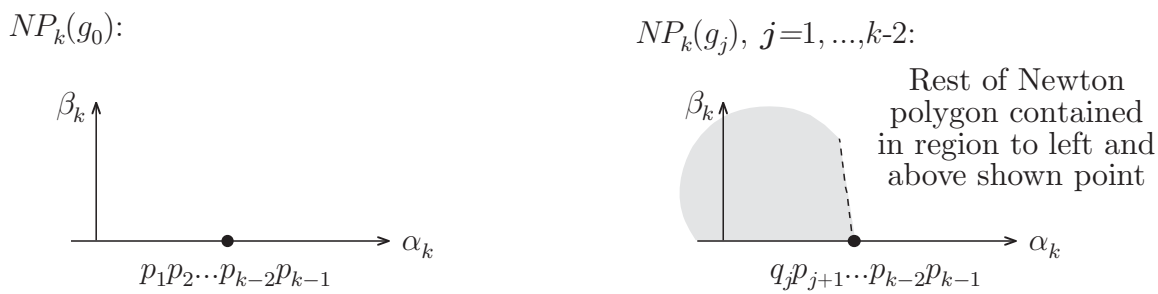

(iii) $\mathrm{NP}_{k}\left(g_{k-1}\right)$ has has right boundary which is a vertical line with corner point $\left(q_{k-1}, 0\right)$.

(iv) $\mathrm{NP}_{k}\left(g_{k}^{p_{k} \cdots p_{h}}\right)$ has corner point $\left(p_{k-1} q_{k-1}, 0\right)$ and the key line segment of $\mathrm{NP}_{k}(f)$ is not parallel to the key line segment of $\mathrm{NP}_{k}\left(g_{k}\right)$ if it exists. 
$N P_{k}\left(g_{k-1}\right)$ :

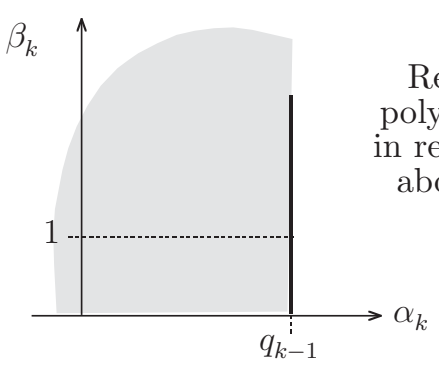

$N P_{k}\left(g_{k}\right)$ :

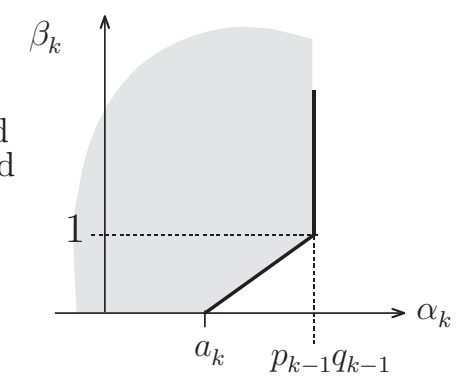

Proof. (i), (ii), and (iii) follow from Lemma 3.18.

To prove (iv), express $g_{k, k-1}$ in the form

$$
c_{k} x_{k-1}^{p_{k-2} q_{k-2} p_{k-1}}\left(y_{k-1}^{p_{k-1}}-t_{k-1}^{p_{k-1}} x_{k-1}^{-\tau_{k-1}}\right)+\sum_{\substack{\alpha_{k-1} p_{k-1}-\beta_{k-1} \tau_{k-1}<q_{k-1} p_{k-1} \\ \alpha_{k-1}}} d_{\alpha_{k-1} \beta_{k-1}}^{\alpha_{k-1}} y_{k-1}^{\beta_{k-1}} .
$$

for some constant $c_{k}$. When we let

$$
\left(x_{k-1}, y_{k-1}\right)=\left(x_{k}^{p_{k-1}}, x_{k}^{-\tau_{k-1}}\left(y_{k}+t_{k-1}\right)\right)
$$

we obtain

$$
\begin{gathered}
g_{k, k}=c_{k} x_{k}^{p_{k-1}\left(p_{k-2} q_{k-2} p_{k-1}-\tau_{k-1}\right)}\left(\left(y_{k}+t_{k-1}\right)^{p_{k-1}}-t_{k-1}^{p_{k-1}}\right) \\
\quad+\sum_{\alpha_{k-1} p_{k-1}-\beta_{k-1} \tau_{k-1}<q_{k-1} p_{k-1}} d_{\alpha_{k-1} \beta_{k-1}} x_{k-1}^{p_{k-1} \alpha_{k-1}-\beta_{k-1} \tau_{k-1}}\left(y_{k}+t_{k-1}\right)^{\beta_{k-1}} .
\end{gathered}
$$

Thus, as $p_{k-2} q_{k-2} p_{k-1}-\tau_{k-1}=q_{k-1}$, the corner point is as required.

Assume that $\mathrm{NP}_{k}\left(g_{k}\right)$ has a key line segment parallel to that of $\mathrm{NP}_{k}(f)$. Note that in this case $\mathrm{NP}_{k}\left(g_{k}\right)$ must include a point on the horizontal axis, $\left(a_{k}, 0\right)$, say. Now compare $\mathrm{NP}_{k}(f)$ with $\mathrm{NP}_{k}\left(g_{k}^{p_{k} \cdots p_{h}}\right)$. The key line segment of $\mathrm{NP}_{k}(f)$ has slope $p_{k} / \tau_{k}$ where $q_{k}=q_{k-1} p_{k-1} p_{k}-\tau_{k}$ and $\operatorname{gcd}\left(p_{k}, \tau_{k}\right)=1$. 


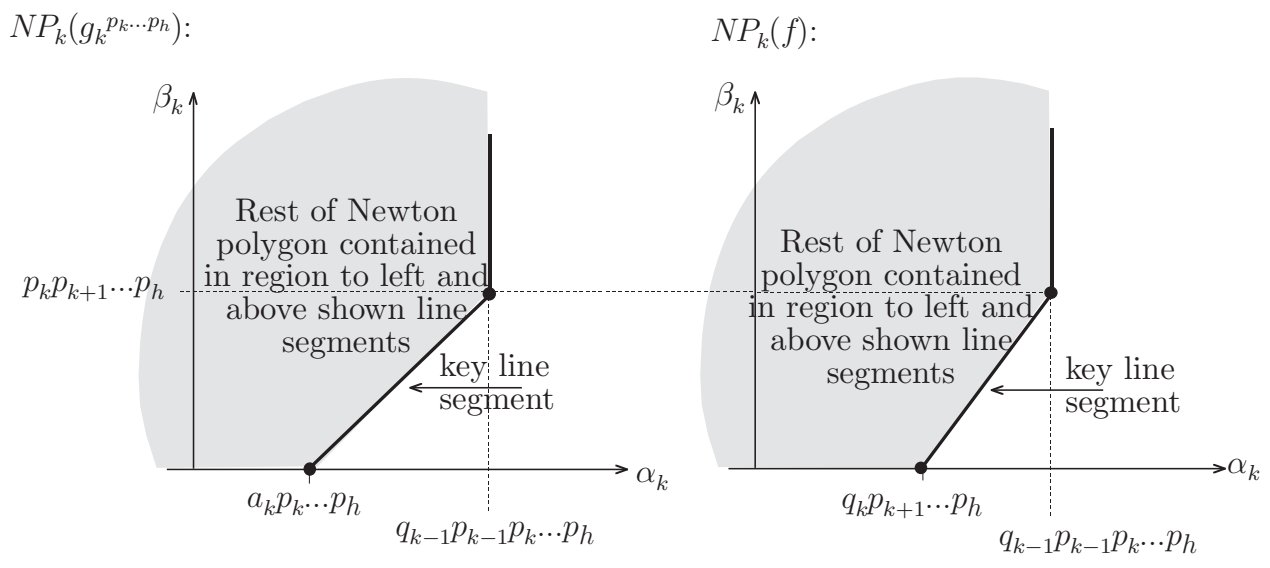

$N P_{k}(f):$

From Corollary 3.12, $\mathrm{NP}_{k}\left(g_{k}^{p_{k} \cdots p_{h}}\right)$ has key line segment with slope $\left(p_{k} p_{k+1} \cdots p_{h}\right) /$ $\left(a_{k} p_{k} p_{k+1} \cdots p_{h}\right)$ and we see that this has the same corner point as $\mathrm{NP}_{k}(f)$. Thus if the the key line segments of $\mathrm{NP}_{k}(f)$ and $\mathrm{NP}_{k}\left(g_{k}^{p_{k} \cdots p_{h}}\right)$ are parallel, they are identical. But then $\tau_{k}=a_{k} p_{k}$ which is not possible because $\operatorname{gcd}\left(p_{k}, \tau_{k}\right)=1$ and $p_{k}>1$.

Corollary 3.20. The corner point of the term $\mathrm{NP}_{k}\left(a_{\gamma_{0} \ldots \gamma_{k}} g_{0}^{\gamma_{0}} \cdots g_{k}^{\gamma_{k}}\right)$ is at $\left(\alpha_{k}, \beta_{k}\right)$ where

$$
\alpha_{k}=\gamma_{0} p_{1} \cdots p_{k-1}+\gamma_{1} q_{1} p_{2} \cdots p_{k-1}+\cdots+\gamma_{k-1} q_{k-1}+\gamma_{k} p_{k-1} q_{k-1}
$$

and

$$
\beta_{k}=\gamma_{k}
$$

Lemma 3.21. If $\mathrm{NP}_{k}\left(g_{k}^{p_{k} \cdots p_{h}}\right)$ has a key line segment, then it has key points on this line segment at

$$
\alpha_{k}=q_{k-1} p_{k-1} \cdots p_{h}-u\left(q_{k-1} p_{k-1}-a_{k}\right), \quad \beta_{k}=p_{k} \cdots p_{h}-u
$$

for some $a_{k} \in \mathbb{C}, u=0, \ldots, p_{k} \cdots p_{h}$.

Proof. Consider $\mathrm{NP}_{k}\left(g_{k}\right)$ which either has no point on the horizontal axis or does have a point on the horizontal axis, at $\left(a_{k}, 0\right)$ say. It is only the latter case where $\mathrm{NP}_{k}\left(g_{k}^{p_{k} \cdots p_{h}}\right)$ will have a key line segment. The key line segment of $\mathrm{NP}_{k}\left(g_{k}\right)$ has end points $\left(q_{k-1} p_{k-1}, 1\right)$ and $\left(a_{k}, 0\right)$ : 


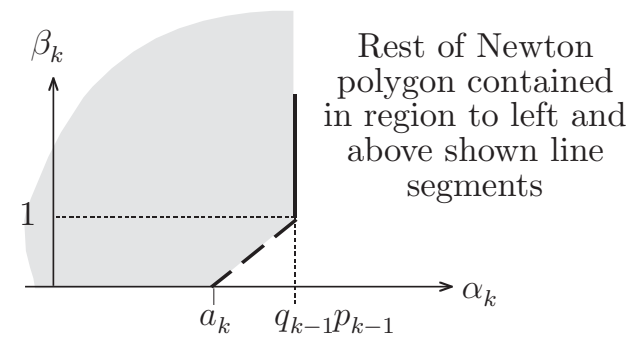

Thus we can express $g_{k}$ in the form

$$
g_{k}=d_{k} x_{k}^{q_{k-1} p_{k-1}}\left(y_{k}-r_{k} x_{k}\right)+\sum_{\alpha_{k}-\beta_{k}\left(q_{k-1} p_{k-1}-a_{k}\right)<a_{k}} d_{\alpha_{k} \beta_{k}} x_{k}^{\alpha_{k}} y_{k}^{\beta_{k}}
$$

for some $d_{k}, d_{\alpha_{k} \beta_{k}}, r_{k} \in \mathbb{C}$ with $d_{k}, r_{k} \neq 0$. It follows that

$$
g_{k}^{p_{k} \cdots p_{h}}=d_{k}^{\prime} x_{k}^{q_{k-1} p_{k-1} p_{k} \cdots p_{h}}\left(y_{k}-r_{k} x_{k}\right)^{p_{k} \cdots p_{h}}+\sum d_{\alpha_{k} \beta_{k}}^{\prime} x_{k}^{\alpha_{k}} y_{k}^{\beta_{k}}
$$

for some $d_{k}^{\prime}, d_{\alpha_{k} \beta_{k}}^{\prime} \in \mathbb{C}$. Therefore, if $\mathrm{NP}_{k}\left(g_{k}^{p_{k} \cdots p_{h}}\right)$ has a key line segment, it has key points at

$$
\left(q_{k-1} p_{k-1} \cdots p_{h}-u\left(q_{k-1} p_{k-1}-a_{k}\right), p_{k} \cdots p_{h}-u\right)
$$

for $u=0,1, \ldots, p_{k} \cdots p_{h}$.

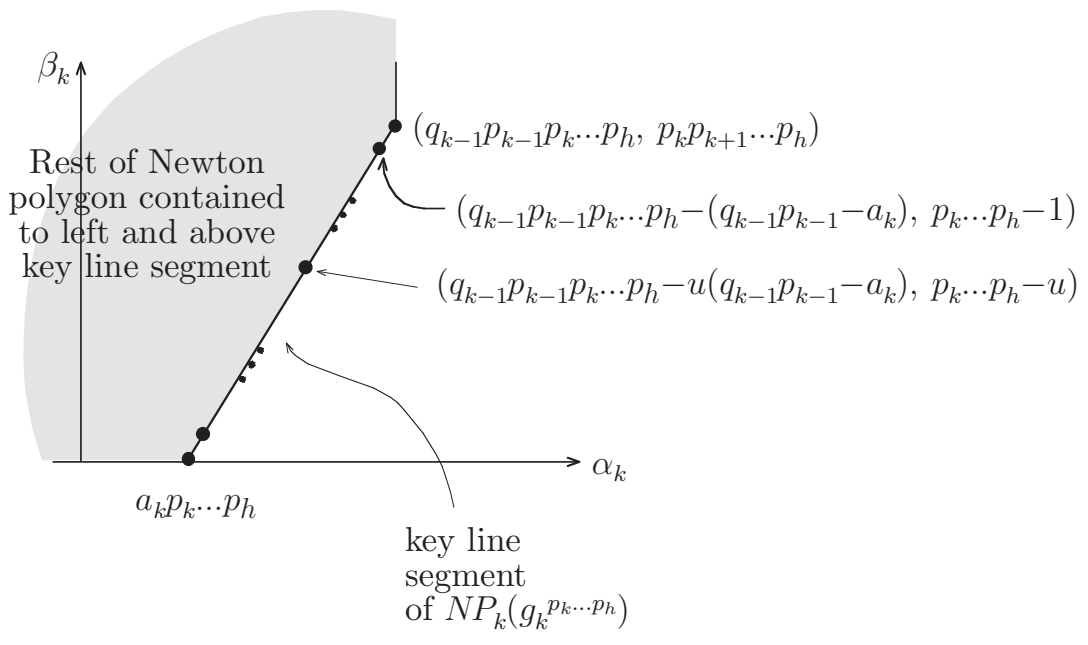


Lemma 3.22. Let $p, q, \phi, \phi^{\prime}, \lambda, \lambda^{\prime} \in \mathbb{Z}$ and assume that

(i) $p \geq 2, q \neq 0$,

(ii) $\operatorname{gcd}(p, q)=1$ and

(iii) $0 \leq \phi, \phi^{\prime}<p$.

If

$$
\lambda p+\phi q=\lambda^{\prime} p+\phi^{\prime} q
$$

then

$$
(\lambda, \phi)=\left(\lambda^{\prime}, \phi^{\prime}\right)
$$

Proof. Without loss of generality, assume $\phi \geq \phi^{\prime}$. Then

$$
p\left(\lambda^{\prime}-\lambda\right)=q\left(\phi-\phi^{\prime}\right)
$$

with $0 \leq \phi-\phi^{\prime}<p$. As $\operatorname{gcd}(p, q)=1, p$ is a factor of $\phi-\phi^{\prime}$. Thus $\phi-\phi^{\prime}=0$ and

$$
\phi=\phi^{\prime} .
$$

Hence

$$
\lambda^{\prime}-\lambda=0
$$

Lemma 3.23. If we have two terms in $f, g_{0}^{\gamma_{0}} g_{1}^{\gamma_{1}} \cdots g_{k}^{\gamma_{k}}$ and $g_{0}^{\gamma_{0}^{\prime}} g_{1}^{\gamma_{1}^{\prime}} \cdots g_{k}^{\gamma_{k}^{\prime}}$ such that

$$
\left(\gamma_{0}, \gamma_{1}, \ldots, \gamma_{k}\right) \neq\left(\gamma_{0}^{\prime}, \gamma_{1}^{\prime}, \ldots, \gamma_{k}^{\prime}\right)
$$

then

$$
\begin{aligned}
& \left(p_{1} \cdots p_{k-1} \gamma_{0}+q_{1} p_{2} \cdots p_{k-1} \gamma_{1}+\cdots+q_{k-1} \gamma_{k-1}+p_{k-1} q_{k-1} \gamma_{k}, \gamma_{k}\right) \\
\neq & \left(p_{1} \cdots p_{k-1} \gamma_{0}^{\prime}+q_{1} p_{2} \cdots p_{k-1} \gamma_{1}^{\prime}+\cdots+q_{k-1} \gamma_{k-1}^{\prime}+p_{k-1} q_{k-1} \gamma_{k}^{\prime}, \gamma_{k}^{\prime}\right) .
\end{aligned}
$$

Proof. We prove the contrapositive. Assume

$$
\begin{aligned}
& \left(p_{1} \cdots p_{k-1} \gamma_{0}+q_{1} p_{2} \cdots p_{k-1} \gamma_{1}+\cdots+q_{k-1} \gamma_{k-1}+p_{k-1} q_{k-1} \gamma_{k}, \gamma_{k}\right) \\
= & \left(p_{1} \cdots p_{k-1} \gamma_{0}^{\prime}+q_{1} p_{2} \cdots p_{k-1} \gamma_{1}^{\prime}+\cdots+q_{k-1} \gamma_{k-1}^{\prime}+p_{k-1} q_{k-1} \gamma_{k}^{\prime}, \gamma_{k}^{\prime}\right) .
\end{aligned}
$$

Then $\gamma_{k}=\gamma_{k}^{\prime}$ and thus

$$
\begin{aligned}
& p_{1} \cdots p_{k-1} \gamma_{0}+q_{1} p_{2} \cdots p_{k-1} \gamma_{1}+\cdots+q_{k-1} \gamma_{k-1} \\
= & p_{1} \cdots p_{k-1} \gamma_{0}^{\prime}+q_{1} p_{2} \cdots p_{k-1} \gamma_{1}^{\prime}+\cdots+q_{k-1} \gamma_{k-1}^{\prime}
\end{aligned}
$$

so that

$$
\begin{aligned}
& p_{k-1}\left(p_{1} \cdots p_{k-2} \gamma_{0}+q_{1} p_{2} \cdots p_{k-2} \gamma_{1}+\cdots+q_{k-2} \gamma_{k-2}\right)+q_{k-1} \gamma_{k-1} \\
= & p_{k-1}\left(p_{1} \cdots p_{k-2} \gamma_{0}^{\prime}+q_{1} p_{2} \cdots p_{k-2} \gamma_{1}^{\prime}+\cdots+q_{k-2} \gamma_{k-2}^{\prime}\right)+q_{k-1} \gamma_{k-1}^{\prime}
\end{aligned}
$$


Then, from Lemma 3.22, as $\operatorname{gcd}\left(p_{k-1}, q_{k-1}\right)=1$ and $0 \leq \gamma_{k-1}, \gamma_{k-1}^{\prime}<p_{k-1}$,

$$
\gamma_{k-1}=\gamma_{k-1}^{\prime}
$$

and

$$
\begin{aligned}
& p_{1} \cdots p_{k-2} \gamma_{0}+q_{1} p_{2} \cdots p_{k-2} \gamma_{1}+\cdots+q_{k-2} \gamma_{k-2} \\
= & p_{1} \cdots p_{k-2} \gamma_{0}^{\prime}+q_{1} p_{2} \cdots p_{k-2} \gamma_{1}^{\prime}+\cdots+q_{k-2} \gamma_{k-2}^{\prime} .
\end{aligned}
$$

Continuing in this fashion we eventually obtain

$$
p_{1} \cdots p_{k-1} \gamma_{0}+q_{1} p_{2} \cdots p_{k-1} \gamma_{1}=p_{1} \cdots p_{k-1} \gamma_{0}^{\prime}+q_{1} p_{2} \cdots p_{k-1} \gamma_{1}^{\prime} .
$$

Then

$$
p_{1} \gamma_{0}+q_{1} \gamma_{1}=p_{1} \gamma_{0}^{\prime}+q_{1} \gamma_{1}^{\prime}
$$

From Lemma 3.22,

$$
\left(\gamma_{0}, \gamma_{1}\right)=\left(\gamma_{0}^{\prime}, \gamma_{1}^{\prime}\right)
$$

and conclude that

$$
\left(\gamma_{0}, \gamma_{1}, \ldots, \gamma_{k}\right)=\left(\gamma_{0}^{\prime}, \gamma_{1}^{\prime}, \ldots, \gamma_{k}^{\prime}\right)
$$

Lemma 3.24. The key line segments of the terms of $f, \mathrm{NP}_{k}\left(a_{\gamma_{0} \gamma_{1} \ldots \gamma_{k}} g_{0}^{\gamma_{0}} g_{1}^{\gamma_{1}} \cdots g_{k}^{\gamma_{k}}\right)$ where $\gamma_{k}>0$

(i) are parallel to each other and are parallel to the key line segment of $\mathrm{NP}_{k}\left(g_{k}^{\left(p_{k} \cdots p_{h}\right)}\right)$ and

(ii) are not parallel to the key line segment of $\mathrm{NP}_{k}(f)$.

Note that if $\gamma_{k}=0, \operatorname{NP}_{k}\left(a_{\gamma_{0} \gamma_{1} \ldots \gamma_{k}} g_{0}^{\gamma_{0}} g_{1}^{\gamma_{1}} \cdots g_{k}^{\gamma_{k}}\right)$ does not have a key line segment and has a corner point on the horizontal axis.

Proof. (i) This follows directly from Corollary 3.13 and Lemma 3.19.

(ii) This follows from Lemmas 3.10 and 3.19 .

Let $h$ be a term of $f$, that is one of $g_{k}^{p_{k} \cdots p_{h}}$ and $a_{\gamma_{0} \ldots \gamma_{k}} g_{0}^{\gamma_{0}} \cdots g_{k}^{\gamma_{k}}$ with $a_{\gamma_{0} \ldots \gamma_{k}} \neq 0$.

Lemma 3.25. Then $\mathrm{NP}_{k}(h)$ does not have any component to the right of $\mathrm{NP}_{k}(f)$. That is, for

$$
h=\sum_{\alpha_{k}, \beta_{k}} b_{\alpha_{k} \beta_{k}} x_{k}^{\alpha_{k}} y_{k}^{\beta_{k}},
$$

the following holds:

$$
\alpha_{k} p_{k}-\beta_{k} \tau_{k} \leq q_{k} p_{k} \cdots p_{h}
$$

and

$$
\alpha_{k} \leq q_{k-1} p_{k-1} \cdots p_{h}
$$


Proof. First we show that $\mathrm{NP}\left(g_{k}^{p_{k} \cdots p_{h}}\right)$ has no component to the right of $\mathrm{NP}_{k}(f)$. There are two cases.

(i) $\mathrm{NP}_{k}\left(g_{k}\right)$ is a vertical line only. In this case it is clear that there is no component to the right of $\mathrm{NP}(f)$.

(ii) $\mathrm{NP}_{k}\left(g_{k}\right)$ is not a vertical line. In this case assume that $\mathrm{NP}\left(g_{k}^{p_{k} \cdots p_{h}}\right)$ does have a component to the right of $\mathrm{NP}_{k}(f)$. Then the key line segments of $\mathrm{NP}_{k}(f)$ and $\mathrm{NP}_{k}\left(g_{k}\right)$ will be as follows:

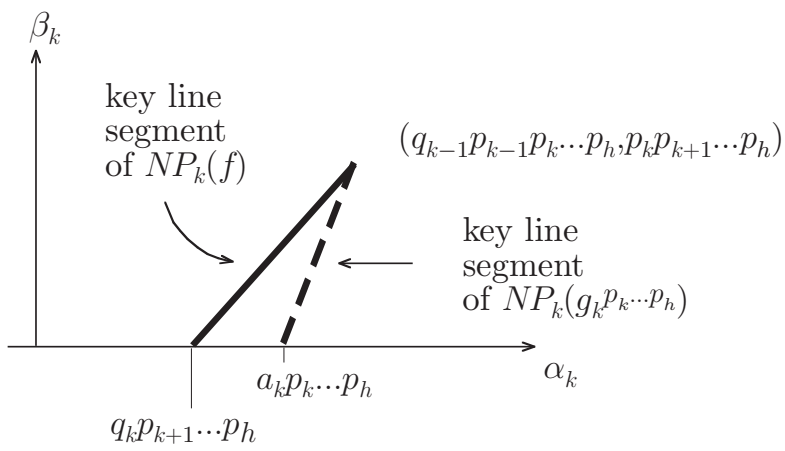

From Lemma 3.21, the key points on the key line segment of $\mathrm{NP}_{k}\left(g_{k}^{p_{k} \cdots p_{h}}\right)$ are

$$
\left(q_{k-1} p_{k-1} p_{k} \cdots p_{h}-u a_{k}, p_{k} \cdots p_{h}-u\right)
$$

with $u=0, \ldots, p_{k} \cdots p_{h}$. (There is a term with non-zero coefficient in $g_{k}^{p_{k} \cdots p_{h}}$ for each of these values.) As these key points lie to the right of $\mathrm{NP}_{k}(f)$, there must be terms in

$$
\zeta_{k}=f-g_{k}^{p_{k} \cdots p_{h}}
$$

having Newton polygons which include the above key points. In particular, there must be one to cancel the term in $g_{k}^{p_{k} \cdots p_{h}}$ corresponding to the key point in $\mathrm{NP}_{k}\left(g_{k}^{p_{k} \cdots p_{h}}\right)$, that is to cancel $\left(q_{k-1} p_{k-1} p_{k} \cdots p_{h}-a_{k}, p_{k} \cdots p_{h}-1\right)$. Consider the terms of $\zeta_{k}, a_{\gamma_{0} \ldots \gamma_{k}} g_{0}^{\gamma_{0}} \cdots g_{k}^{\gamma_{k}}$. As $\gamma_{k}<p_{k} \cdots p_{h}-1$, it follows that there is no $\mathrm{NP}_{k}\left(a_{\gamma_{0} \ldots \gamma_{k}} g_{0}^{\gamma_{0}} \cdots g_{k}^{\gamma_{k}}\right)$ whose corner point is $\left(q_{k-1} p_{k-1} p_{k} \cdots p_{h}-a_{k}, p_{k} \cdots p_{h}-1\right)$. Thus there is a term $a_{\gamma_{0} \ldots \gamma_{k}} g_{0}^{\gamma_{0}} \cdots g_{k}^{\gamma_{k}}$ with non-zero coefficient with corner point lying beyond the right side of $\mathrm{NP}_{k}\left(g_{k}^{p_{k} \cdots p_{h}}\right)$, that is, at some $\left(\alpha_{k}^{\prime}, \beta_{k}^{\prime}\right)$ with $p_{k} \alpha_{k}^{\prime}-a_{k} p_{k} \beta_{k}^{\prime}>$ $p_{k} \alpha_{k}-a_{k} p_{k} \beta_{k}$ 


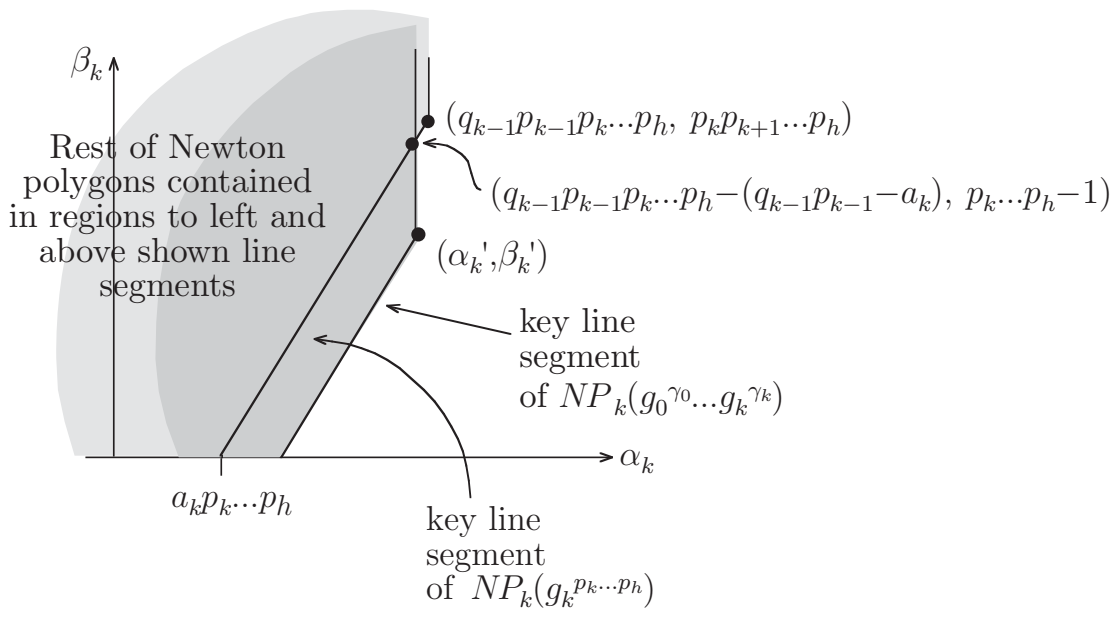

Take the terms $a_{\gamma_{0} \ldots \gamma_{k}} g_{0}^{\gamma_{0}} \cdots g_{k}^{\gamma_{k}}$ with $a_{\gamma_{0} \ldots \gamma_{k}} \neq 0$ such that $p_{k} \alpha_{k}-a_{k} p_{k} \beta_{k}$ is maximum for the corner points, $\left(\alpha_{k}, \beta_{k}\right)$, of $\mathrm{NP}_{k}\left(a_{\gamma_{0} \cdots \gamma_{k}} g_{0}^{\gamma_{0}} \cdots g_{k}^{\gamma_{k}}\right)$. From these choose that term whose corner point has maximum $\beta_{k}$.

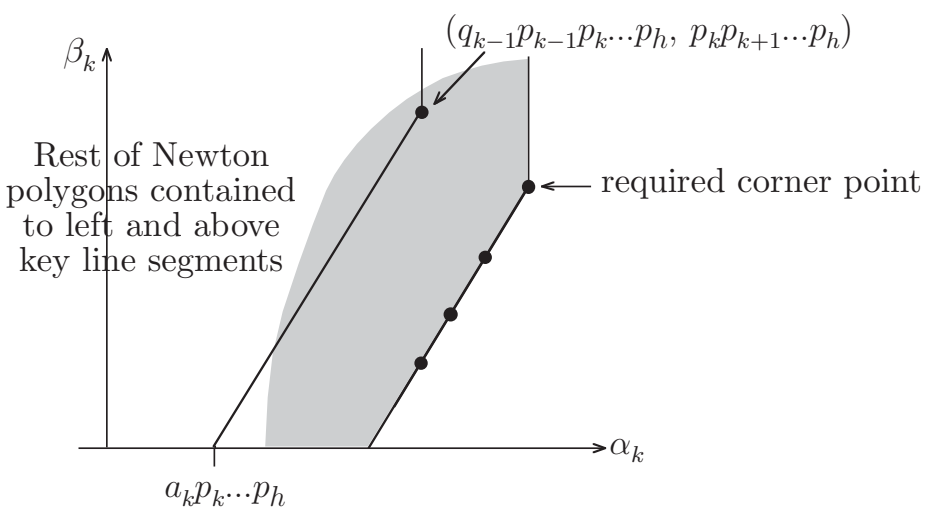

No other term can have a Newton polygon with the same corner point (Lemma 3.23). By construction, this corner point does not lie within the Newton polygon of any other $a_{\gamma_{0} \ldots \gamma_{k}} g_{0}^{\gamma_{0}} \cdots g_{k}^{\gamma_{k}}$. Thus this corner point must lie in $\operatorname{NP}_{k}(f)$. But this is a contradiction as we assumed that $\mathrm{NP}_{k}(f)$ has key line segment to the left of the key line segment of $\mathrm{NP}_{k}\left(g_{k}^{p_{k} \cdots p_{h}}\right)$. Hence the key line segment of $\mathrm{NP}_{k}\left(g_{k}^{p_{k} \cdots p_{h}}\right)$ and thus its corner point lie inside $\mathrm{NP}_{k}(f)$.

Secondly, we assume that there is some term $a_{\gamma_{0} \ldots \gamma_{k}} g_{0}^{\gamma_{0}} \cdots g_{k}^{\gamma_{k}}$ in $\zeta_{k}$, with Newton polygon whose corner point lies to the right of $\mathrm{NP}_{k}(f)$. A similar construction to that used above leads to a contradiction and the result follows from the fact that any key line segment of $\mathrm{NP}_{k}\left(a_{\gamma_{0} \ldots \gamma_{k}} g_{0}^{\gamma_{0}} \cdots g_{k}^{\gamma_{k}}\right)$ is parallel to that of $\mathrm{NP}_{k}\left(g_{k}^{p_{k} \cdots p_{h}}\right)$ (Lemma 3.24). 
Lemma 3.26. The polynomial

$$
f=g_{k}^{p_{k} \cdots p_{h}}+\sum a_{\gamma_{0} \ldots \gamma_{k}} g_{0}^{\gamma_{0}} \cdots g_{k}^{\gamma_{k}}
$$

with $0 \leq \gamma_{0}, 0 \leq \gamma_{1}<p_{1}, \ldots, 0 \leq \gamma_{k-1}<p_{k-1}, 0 \leq \gamma_{k}<p_{k} \cdots p_{h}-1$, is such that the following terms have non-zero coefficients:

$$
\begin{aligned}
& \left\{a_{\gamma_{0} \cdots \gamma_{k}} g_{0}^{\gamma_{0}} \cdots g_{k}^{\gamma_{k}} \mid\right. \\
& \quad\left(p_{1} p_{2} \cdots p_{k-1} \gamma_{0}+q_{1} p_{2} \cdots p_{k-1} \gamma_{1}+q_{2} p_{3} \cdots p_{k-1} \gamma_{2}+\cdots+\gamma_{k}, \gamma_{k}\right) \\
& \left.\quad=\left(q_{k-1} p_{k-1} \cdots p_{h}-s \tau_{k}, p_{k} \cdots p_{h}-s p_{k}\right), \quad s=1,2, \ldots, p_{k+1} \cdots p_{h}\right\}
\end{aligned}
$$

Proof. Recall that $\mathrm{NP}_{k}\left(g_{k}^{p_{k} \cdots p_{h}}\right)$ and $\left\{\mathrm{NP}_{k}\left(a_{\gamma_{0} \ldots \gamma_{k}} g_{0}^{\gamma_{0}} \cdots g_{k}^{\gamma_{k}}\right) \mid a_{\gamma_{0} \ldots \gamma_{k}} \neq 0\right\}$

(i) have unique corner points (Lemma 3.23),

(ii) either have no key line segment or a key line segment which is not parallel to the key line segment of $\mathrm{NP}_{k}(f)$ (Lemma 3.19),

(iii) have corner points which lie within $\mathrm{NP}_{k}(f)$ (Lemma 3.25) and

(iv) $f_{k}$ has a term with non-zero coefficient for each key point

$$
\alpha_{k}=q_{k-1} p_{k-1} \cdots p_{h}-s \tau_{k} \quad \text { and } \quad \beta_{k}=p_{k} \cdots p_{h}-s p_{k}
$$

on the key line segment of $\mathrm{NP}_{k}(f)$ (Lemma 3.10).

Thus there must be terms $a_{\gamma_{0} \ldots \gamma_{k}} g_{0}^{\gamma_{0}} \cdots g_{k}^{\gamma_{k}}$ with non-zero coefficients whose corner points match the key points on the key line segment of $f$ :

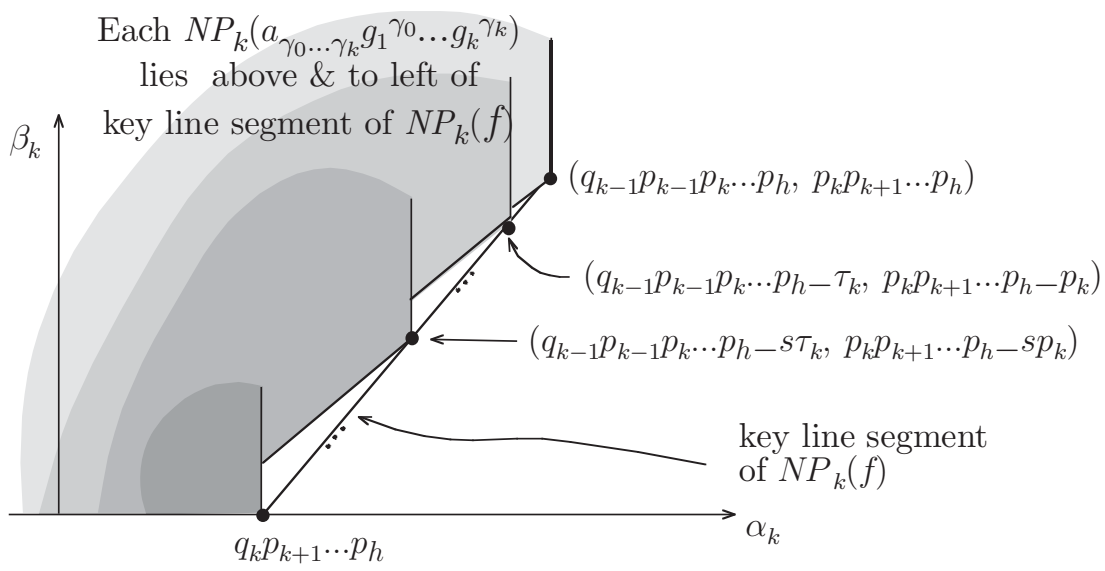


Lemma 3.27. Assume that for $j=1, \ldots, h-k, \mathrm{NP}_{k-1}\left(g_{k+j}^{p_{k+j} \cdots p_{h}}\right)$ has the same same key line segment as $\mathrm{NP}_{k-1}(f)$ for $i=1, \ldots, k-1$ and the terms in $g_{k+j, i}^{p_{k+1} \cdots p_{h}}$ and $f_{i}$ corresponding to this key line segment are the same.

Then $\mathrm{NP}_{k}\left(g_{k+j}^{p_{k+j} \cdots p_{h}}\right)$ lies inside $\mathrm{NP}_{k}(f)$.

Proof. We note that the right vertical line segment in $\mathrm{NP}_{k}\left(g_{k+j}^{p_{k+j} \cdots p_{k}}\right)$ is the same as the right vertical line segment of $\mathrm{NP}_{k}(f)$ (because $\mathrm{NP}_{k-1}\left(g_{k+j}^{p_{k+j} \cdots p_{h}}\right)$ has the same same key line segment as $\mathrm{NP}_{k-1}(f)$ for $i=1, \ldots, k-1$ and the terms in $g_{k+j, i}^{p_{k+1} \cdots p_{h}}$ and $f_{i}$ corresponding to this key line segment are the same).

As $g_{k+j}$ is the $\left(p_{k+j} \cdots p_{h}\right)^{\text {th }}$ approximate root of $f$,

$$
f=g_{k+j}^{p_{k+j} \cdots p_{h}}+\zeta_{k+j}
$$

where $\operatorname{deg}_{y}\left(\zeta_{k+j}\right)<p_{1} \cdots p_{h}-p_{1} \cdots p_{k-1+j}$. Using the techniques of Lemma 3.9 we have that

$$
\zeta_{k+j}=\sum b_{\eta_{0} \ldots \eta_{k}} g_{0}^{\eta_{0}} \cdots g_{k}^{\eta_{k}}
$$

with $\eta_{1}<p_{1}, \ldots, \eta_{k-1}<p_{k-1}, \eta_{k}<p_{k} \cdots p_{h}-p_{k} \cdots p_{k-1+j}$. Assume that $\mathrm{NP}_{k}\left(g_{k+j}\right)$ has a point on its boundary, $\left(\alpha_{k}^{\prime}, \beta_{k}^{\prime}\right)$ such that

- $\alpha_{k}^{\prime} p_{k}-\beta_{k}^{\prime} \tau_{k}>q_{k} p_{k} \cdots p_{k+j-1}$ is the maximum possible value and

- $\beta_{k}^{\prime}$ is the maximum for the key points on this line segment.

Then there is a line segment in the boundary of $\mathrm{NP}_{k}\left(g_{k+j}\right)$ which includes the key points

$$
\left(q_{k-1} p_{k-1} p_{k} \cdots p_{k+j-1}, p_{k} \cdots p_{k+j-1}\right)
$$

and

$$
\left(\alpha_{k}^{\prime}, \beta_{k}^{\prime}\right)
$$

with no other key points in between. Thus, from Lemma $3.14, \mathrm{NP}_{k}\left(g_{k+j}^{p_{k+j} \cdots p_{h}}\right)$ includes the key point $\left(\alpha_{k}, \beta_{k}\right)$ where

$$
\alpha_{k}=q_{k-1} p_{k-1} p_{k} \cdots p_{h}-\left(q_{k-1} p_{k-1} p_{k} \cdots p_{k+j-1}-\alpha_{k}^{\prime}\right)
$$

and

$$
\beta_{k}=p_{k} \cdots p_{h}-\left(p_{k} \cdots p_{k+j-1}-\beta_{k}^{\prime}\right) .
$$

This point lies outside $\mathrm{NP}_{k}(f)$ as

$$
\begin{aligned}
p_{k}\left(q_{k-1} p_{k-1} p_{k} \cdots p_{h}-\left(q_{k-1} p_{k-1} p_{k}\right.\right. & \left.\left.\cdots p_{k+j-1}-\alpha_{k}^{\prime}\right)\right) \\
& -\tau_{k}\left(p_{k} \cdots p_{h}-\left(p_{k} \cdots p_{k+j-1}-\beta_{k}^{\prime}\right)\right) \\
= & q_{k} p_{k} \cdots p_{h}-q_{k} p_{k} \cdots p_{k+j-1}+\alpha_{k}^{\prime} p_{k}-\tau_{k} \beta_{k}^{\prime} \\
> & q_{k} p_{k} \cdots p_{h}
\end{aligned}
$$


Thus there must be some $b_{\eta_{0} \ldots \eta_{k}} g_{0}^{\eta_{0}} \cdots g_{k}^{\eta_{k}}$ in $\zeta_{k+j}$ which cancels this term in $g_{k+1}^{p_{k+j} \cdots p_{h}}$ and therefore a term in $\zeta_{k+1}$ with corner point of $\mathrm{NP}_{k}\left(b_{\eta_{0} \ldots \eta_{k}} g_{0}^{\eta_{0}} \cdots g_{k}^{\eta_{k}}\right)$ lying outside $\mathrm{NP}_{k}(f)$. If we do the construction as in the proof of Lemma 3.25 we will get a contradiction in exactly the same way.

Scholium 3.28. The terms of $\zeta_{k+j}, b_{\eta_{0} \ldots \eta_{k}} g_{0}^{\eta_{0}} \cdots g_{k}^{\eta_{k}}$, are such that the corner points of $\mathrm{NP}_{k}\left(b_{\eta_{0} \ldots \eta_{k}} g_{0}^{\eta_{0}} \cdots g_{k}^{\eta_{k}}\right)$ are inside $\mathrm{NP}_{k}(f)$ and contained within the region $\alpha_{k} \leq$ $q_{k-1} p_{k-1} \cdots p_{h}-\tau_{k} p_{k+1} \cdots p_{k+j-1}$.

Proof. From the last part of the proof of the previous result we see that $\mathrm{NP}_{k}\left(\zeta_{k+j}\right)$ has terms, $\quad b_{\eta_{0} \ldots \eta_{k}} g_{0}^{\eta_{0}} \cdots g_{k}^{\eta_{k}}$, with corner points lying within $\mathrm{NP}_{k}(f)$ and with $\eta_{k}<p_{k} \cdots p_{h}-p_{k} \cdots p_{k+j-1}$. The result follows.

Lemma 3.29. For $j=1, \ldots, h-k, \mathrm{NP}_{k}\left(g_{k+j}^{p_{k+j} \cdots p_{h}}\right)$ has the same key line segment as $\mathrm{NP}_{k}(f)$ and the terms in $g_{k+j, k}^{p_{k+j} \cdots p_{h}}$ and $f_{k}$ corresponding to these are identical.

Proof. As $\mathrm{NP}_{k}\left(g_{k+j}^{p_{k+j} \cdots p_{h}}\right)$ is contained in $\mathrm{NP}_{k}(f), \mathrm{NP}_{k}\left(g_{k+j}\right)$ is contained in the region

$$
\begin{aligned}
p_{k} \alpha_{k}-\tau_{k} \beta_{k} & \leq q_{k-1} p_{k-1} \cdots p_{k+j-1}-\tau_{k} p_{k+1} \cdots p_{k+j-1} \\
& =q_{k} p_{k} \cdots p_{k+j-1}
\end{aligned}
$$

as shown:

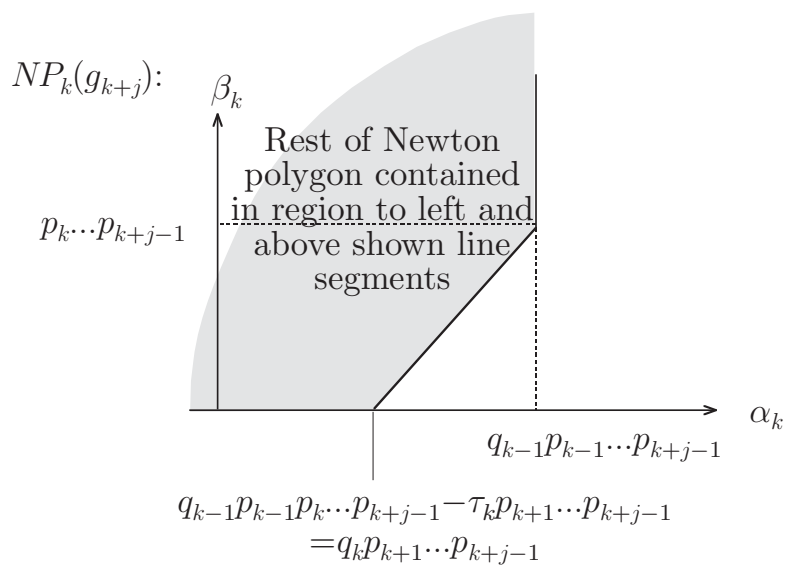

We have

$$
g_{k+j, k}=x_{k}^{q_{k-1} p_{k-1} \cdots p_{k+j-1}} \sum_{i=0}^{p_{k+1} \cdots p_{k+j-1}} d_{i}\left(x_{k}^{-\tau_{k}}\right)^{i}\left(y_{k}^{p_{k}}\right)^{\left(p_{k+1} \cdots p_{k+j-1}-i\right)}+\sum_{p_{k} \alpha_{k}-\tau_{k} \beta_{k}<q_{k-1} p_{k-1} \cdots p_{k+j-1}} d_{\alpha_{k} \beta_{k}} x_{k}^{\alpha_{k}} y_{k}^{\beta_{k}}
$$


for some $d_{i}, d_{\alpha \beta} \in \mathbb{C}$. Take the $\left(p_{k+j} \cdots p_{h}\right)^{\text {th }}$ power of this to obtain

$$
\begin{array}{r}
g_{k+1}^{p_{k+j} \cdots p_{h}}=x_{k}^{q_{k-1} p_{k-1} \cdots p_{h}}\left(\sum_{i=0}^{p_{k+1} \cdots p_{k+j-1}} d_{i}\left(x_{k}^{-\tau_{k}}\right)^{i}\left(y_{k}^{p_{k}}\right)^{\left(p_{k+1} \cdots p_{k+j-1}-i\right)}\right)^{p_{k+1} \cdots p_{h}} \\
+\sum_{p_{k} \alpha_{k}-\tau_{k} \beta_{k}<q_{k-1} p_{k-1} \cdots p_{h}} d_{\alpha_{k} \beta_{k}}^{\prime} x_{k}^{\alpha_{k}} y_{k}^{\beta_{k}} .
\end{array}
$$

Equate $g_{k+j, k}^{p_{k+j} \cdots p_{h}}+\zeta_{k+j, k}$ to obtain

$$
\begin{aligned}
f_{k}= & d_{0} x_{k}^{q_{k-1} p_{k-1} \cdots p_{h}}\left(y_{k}^{p_{k}}-t_{k}^{p_{k+1}} x_{k}^{-\tau_{k}}\right)^{\left(p_{k+1} \cdots p_{k+j-1}\right)\left(p_{k+j} \cdots p_{h}\right)}+\sum_{p_{k} \alpha_{k}-\tau_{k} \beta_{k}<q_{k-1} p_{k-1} \cdots p_{h}} a_{\alpha \beta}^{\prime} x_{k}^{\alpha_{k}} y_{k}^{\beta_{k}} \\
= & d x_{k}^{q_{k-1} p_{k-1} \cdots p_{h}} \\
& \cdot\left(\sum_{m=0}^{p_{k+1} \cdots p_{k+j-1}}\left(\begin{array}{c}
p_{k+1} \cdots p_{k+j-1} \\
m
\end{array}\right) y_{k}^{p_{k}\left(p_{k+1} \cdots p_{k+j-1}-m\right)}\left(t_{k}^{p_{k+1}} x^{-\tau_{k}}\right)^{m}\right)^{p_{k+j} \cdots p_{h}} \\
& +\sum_{p_{k} \alpha_{k}-\tau_{k} \beta_{k}<q_{k-1} p_{k-1} \cdots p_{h}} a_{\alpha \beta}^{\prime} x_{k}^{\alpha_{k}} y_{k}^{\beta_{k}}
\end{aligned}
$$

From Scholium 3.28, $\zeta_{k+j, k}$ does not have any terms $x_{k}^{\alpha_{k}} y_{k}^{\beta_{k}}$ with non-zero coefficient satisfying

$$
\alpha_{k}>q_{k-1} p_{k-1} \cdots p_{h}-\tau_{k} p_{k+1} \cdots p_{k+j-1} .
$$

Thus we see that the terms in (1) and (2) with degree in $y_{k}>q_{k-1} p_{k-1} \cdots p_{h}-$ $\tau_{k} p_{k+1} \cdots p_{k+j-1}$ satisfying $p_{k} \alpha_{k}-\tau_{k} \beta_{k}=q_{k-1} p_{k-1} \cdots p_{h}$ correspond. Thus

$$
\begin{aligned}
d_{0} y_{k}^{p_{k} \cdots p_{k+j-1}\left(p_{k+j} \cdots p_{h}\right)} & \\
& +\left(p_{k+j} \cdots p_{h}\right) d_{0} y_{k}^{p_{k} \cdots p_{k+j-1}\left(p_{k+j} \cdots p_{h}-1\right)} \sum_{i=1}^{p_{k+1} \cdots p_{k+j-1}} d_{i} x_{k}^{-\tau_{k} i} y_{k}^{p_{k}\left(p_{k+1} \cdots p_{k+j-1}-i\right)} \\
= & d y_{k}^{p_{k} \cdots p_{h}}+d\left(p_{k+j} \cdots p_{h}\right) y_{k}^{p_{k} \cdots p_{k+j-1}\left(p_{k+j} \cdots p_{h}-1\right)} \\
& \cdot \sum_{i=1}^{p_{k+1} \cdots p_{k}}\left(\begin{array}{c}
p_{k+j} \cdots p_{k+j-1} \\
i
\end{array}\right)\left(t_{k}^{p_{k}} x_{k}^{-\tau_{k}}\right)^{i} y_{k}^{p_{k}\left(p_{k+1} \cdots p_{h}-i\right)} .
\end{aligned}
$$

This uniquely determines the $d_{i}$ in terms of $d$ and $t_{k}^{p_{k}}$ and we get the required values for each $d_{i}$.

\subsection{The case of redundant cables}

In the previous part of this section we assumed that the minimal splice diagram was the same as the original splice diagram. However this is not always the case as we 
may have Newton pairs of the form $(1, s)$ which lead to redundant cables as we build up the link at infinity. To allow for this we replace the assumptions of Section 3.3 by the following:

(i) $f \in \mathbb{C}^{2}$ is a polynomial with $f(x, y)=0$ defining an irreducible curve $C$ with one place at infinity.

(ii) $f$ has minimal splice diagram:

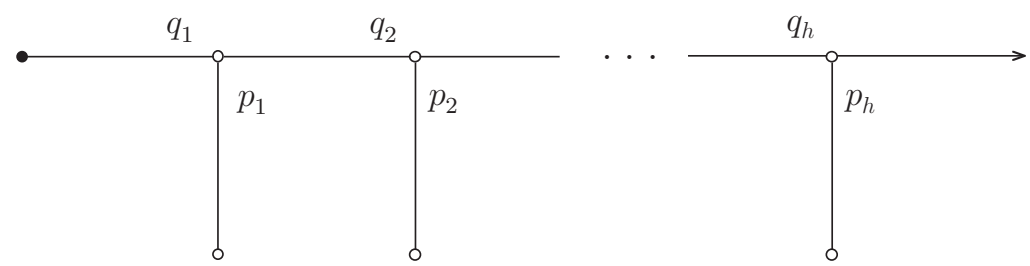

(iii) The cabling pairs of $f$ include (in the given order)

$$
\left(p_{1}, q_{1}\right),\left(p_{2}, q_{2}\right), \ldots,\left(p_{h}, q_{h}\right)
$$

with $p_{i}>1$ for all $i$ while the Newton pairs include

$$
\left(p_{1}, q_{1}\right),\left(p_{2},-\tau_{2}\right), \ldots,\left(p_{h},-\tau_{h}\right) .
$$

However for one or more values of $k, k>1$, we find additional Newton pairs

$$
\left(1,-\omega_{k, 1}\right),\left(1,-\omega_{k, 2}\right), \ldots,\left(1,-\omega_{k, l_{k}}\right)
$$

between $\left(p_{k-1},-\tau_{k-1}\right)$ and $\left(p_{k},-\tau_{k}\right)$ which lead to redundant cabling pairs

$$
\left(1, s_{k, 1}\right),\left(1, s_{k, 2}\right), \ldots,\left(1, s_{k, l_{k}}\right) .
$$

The relevant part of the non-reduced splice diagram is

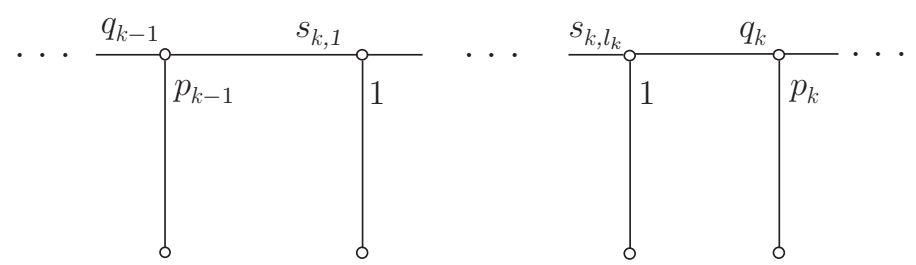

(iv) In order to find the cabling pairs of $f$

$$
\left(p_{1}, q_{1}\right),\left(p_{2}, q_{2}\right), \ldots,\left(p_{h}, q_{h}\right)
$$


we make the following substitutions. If there are no additional Newton pairs between $\left(p_{k-1},-\tau_{k-1}\right)$ and $\left(p_{k},-\tau_{k}\right)$,

$$
\Phi_{j}:\left(x_{k-1}, y_{k-1}\right)=\left(x_{k}^{p_{k-1}}, x_{k}^{-\tau_{k-1}}\left(y_{k}+t_{k-1}\right)\right)
$$

as before. Where additional Newton pairs occur between $\left(p_{k-1},-\tau_{k-1}\right)$ and $\left(p_{k},-\tau_{k}\right)$ we make the following substitutions:

- $\Phi_{k-1}:\left(x_{k-1}, y_{k-1}\right)=\left(x_{k, 1}^{p_{k-1}}, x_{k, 1}^{-\tau_{k-1}}\left(y_{k, 1}+t_{k-1}\right)\right)$. Thus $s_{k, 1}=q_{k-1} p_{k-1}-$ $\tau_{k-1}$.

- $\Omega_{k, j}:\left(x_{k, j}, y_{k, j}\right)=\left(x_{k, j+1}, x_{k, j+1}^{-\omega_{k, j}}\left(y_{k, j+1}+u_{k, j}\right)\right)$ for $j=1, \ldots, k_{l}-1$ giving $s_{k, j}=s_{k, j-1}-\omega_{k, j}$.

- $\Omega_{k, l_{k}}:\left(x_{k, l_{k}}, y_{k, l_{k}}\right)=\left(x_{k}, x_{k}^{-\omega_{k, l_{k}}}\left(y_{k}+u_{k, l_{k}}\right)\right)$ giving $q_{k}=s_{k, l_{k}} p_{k}-\tau_{k}$.

For convenience we call the images of $f$ used at the stages of finding the cabling pairs $\left(p_{1}, q_{1}\right),\left(p_{2}, q_{2}\right), \ldots,\left(p_{h}, q_{h}\right)$, by $f_{1}, \ldots, f_{h}$ as before. We denote the image of $f$ used to determine $\Omega_{k_{j}}$ by $f_{k, j}$ and the corresponding Newton polygon as $\mathrm{NP}_{k, j}(f)$. We carry through the notation of the images and Newton polygons of each $g_{i}$ under the actions of $\Phi_{i}$ and $\Omega_{k, j}$ in the same way.

We obtain the following Newton polygon after applying $\Phi_{k-1}$ :

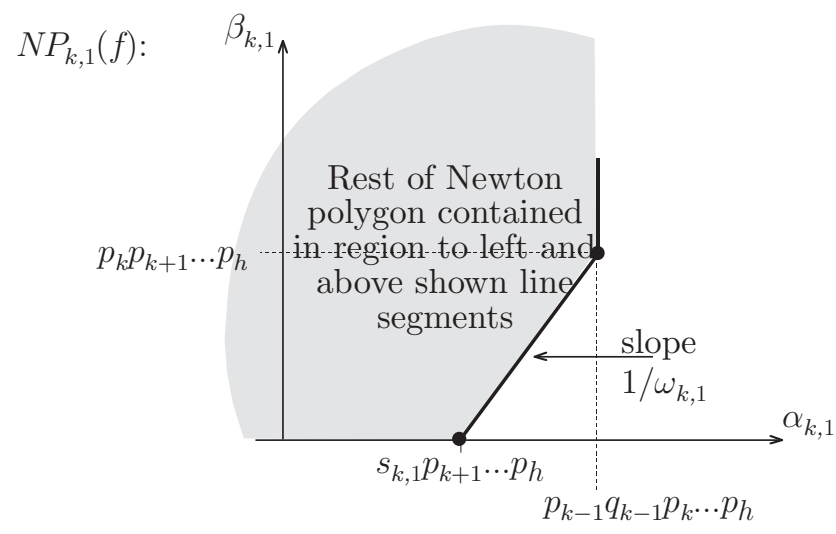


After applying $\Omega_{k, 1}, \ldots, \Omega_{k, l_{k}-1}$ we obtain

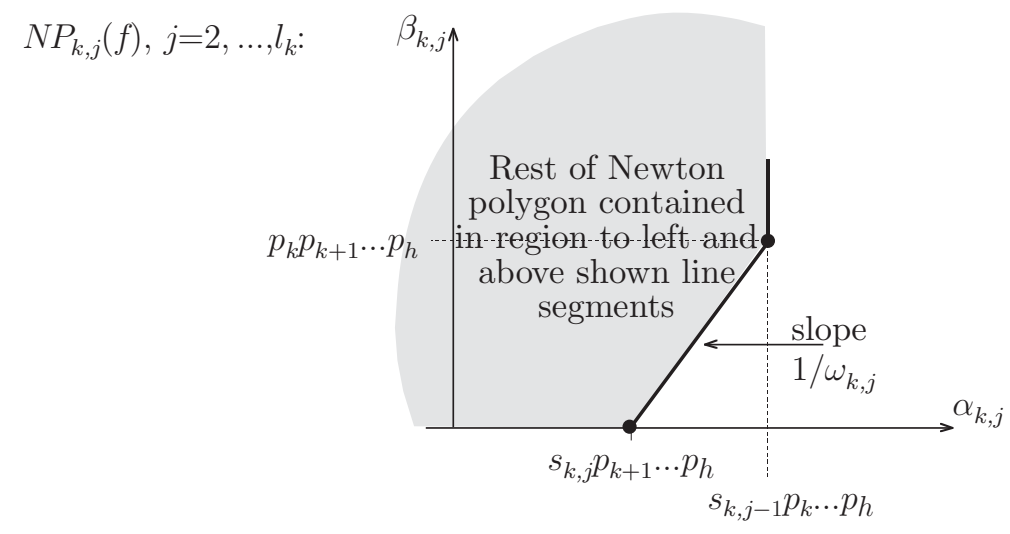

Finally we apply $\Omega_{k, l_{k}}$ to get

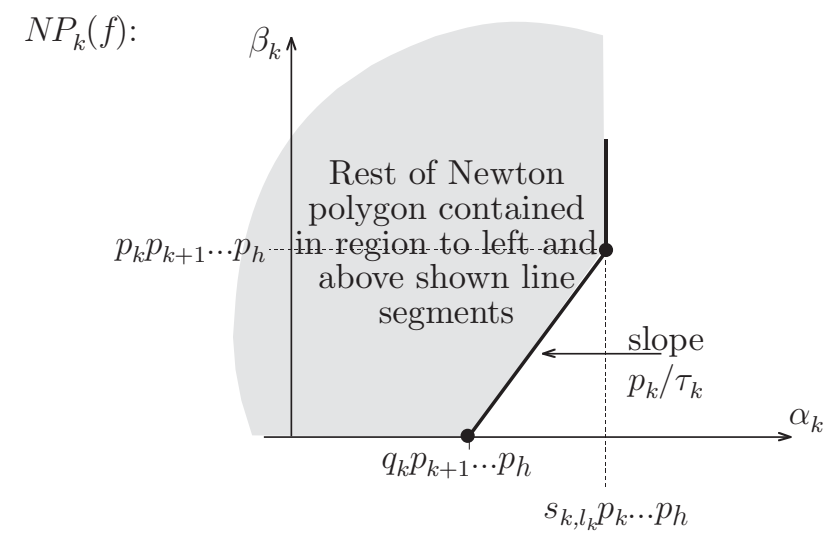

Lemma 3.30. For $i=0, \ldots, k-1$, the corner points of $\mathrm{NP}_{k, j}\left(g_{i}\right)$ are the same as the corner points of $\mathrm{NP}_{k}\left(g_{i}\right), j=1, \ldots, l_{k}$, which are the same corner points as given in Lemma 3.19, that is,

(i) $\mathrm{NP}_{k}\left(g_{0}\right)$ and $\mathrm{NP}_{k, j}\left(g_{0}\right)=\mathrm{NP}_{k, j}(x)$ are points on the horizontal axes, $\left(p_{1} p_{2} \cdots p_{k-1}, 0\right)$.

(ii) $\mathrm{NP}_{k}\left(g_{i}\right)$ and $\mathrm{NP}_{k, j}\left(g_{i}\right), i=1,2, \ldots, k-2$ have corner points, $\left(q_{i} p_{i+1} \cdots p_{k-1}, 0\right)$.

(iii) $\mathrm{NP}_{k}\left(g_{k-1}\right)$ and $\mathrm{NP}_{k, j}\left(g_{k-1}\right)$ have corner points $\left(q_{k-1}, 0\right)$ lying on the horizontal axis. 
Proof. Initially we apply $\Phi_{k-1}$ as in Lemma 3.19, so that the result is true for $j=1$. When we apply $\Omega_{k, j}$, the term corresponding to the corner point of $\operatorname{NP}_{k, j}\left(g_{i}\right)$, is of the form $c x_{k, j}^{\alpha}$ and so maps to $c x_{k, j+1}^{\alpha}$ as required.

Lemma 3.31. For $i=0, \ldots, h-k$ and $\mathrm{NP}_{k}\left(g_{k+i}\right)$ and $\mathrm{NP}_{k, j}\left(g_{k+i}^{p_{k+i} \cdots p_{h}}\right)$, $j=1, \ldots, l_{k}$, have the same key line segments as $\mathrm{NP}_{k}(f)$ and $\mathrm{NP}_{k, j}(f)$ respectively and the terms in $g_{k+i, k, j}^{p_{k+i} \cdots p_{h}}$ and $f_{k, j}$ corresponding to these are identical.

Proof. (i)

$$
f_{k, 1}=c_{1} x_{k, 1}^{q_{k-1} p_{k-1} \cdots p_{h}}\left(y_{k, 1}-u_{k, 1} x_{k, 1}^{-\omega_{k, 1}}\right)^{p_{k} \cdots p_{h}}+\sum_{\alpha_{k, 1}-\omega_{k, 1} \beta_{k, 1}<s_{1} p_{k} \cdots p_{h}} d_{\alpha_{k, 1} \beta_{k, 1}} x_{k, 1}^{\alpha_{k, 1}} y_{k, 1}^{\beta_{k, 1}}
$$

and

$$
f_{k, j}=c_{j} x_{k, j}^{s_{j-1} p_{k} \cdots p_{h}}\left(y_{k, j}-u_{k, j} x_{k, j}^{-\omega_{k, j}}\right)^{p_{k} \cdots p_{h}}+\sum_{\alpha_{k, j}-\omega_{k, j} \beta_{k, j}<s_{k, j} p_{k} \cdots p_{h}} d_{\alpha_{k, j} \beta_{k, j}} x_{k, j}^{\alpha_{k, j}} y_{k, j}^{\beta_{k, j}}
$$

for $j=2, \ldots, l_{k}, f_{k, j}$ has a term, $x_{k, j}^{s_{j} p_{k} \cdots p_{h}-\omega_{k, j}} y^{p_{k} \cdots p_{h}-1}$, with non-zero coefficient.

(ii) By the arguments in the proofs of Lemmas 3.27 and 3.25 the right boundary of $\mathrm{NP}_{k, j}\left(g_{k+i}^{p_{k+i} \cdots p_{h}}\right)$, lies inside $\mathrm{NP}_{k, j}(f)$ for $i=0, \ldots, h-k$.

Given these two items we can use the same argument as Lemma 3.29 to obtain the desired result.

Lemmas 3.31 and 3.30 ensure that the results proved in the technical lemmas needed to prove the Abhyankar-Moh semi-group theorem still hold, that is,

(i) that the terms of $f$ with non-zero coefficients have Newton polygons with corner points occurring at $\left(\alpha_{k}, \beta_{k}\right)$ where

$$
\begin{aligned}
\alpha_{k} & =p_{1} \cdots p_{k-1} \gamma_{0}+q_{1} p_{2} \cdots p_{k-1} \gamma_{1}+\cdots+q_{k-1} \gamma_{k-1}+s_{k, l_{k}} \gamma_{k} \\
\beta_{k} & =\gamma_{k}
\end{aligned}
$$

(ii) that there is some term in $f$

$$
a_{\gamma_{0}^{\prime} \ldots \gamma_{k}^{\prime}} g_{0}^{\gamma_{0}^{\prime}} \cdots g_{k}^{\gamma_{k}^{\prime}}
$$

$a_{\gamma_{0}^{\prime} \ldots \gamma_{k}^{\prime}} \neq 0$ whose Newton polygon $\mathrm{NP}_{k}\left(a_{\gamma_{0}^{\prime} \ldots \gamma_{k}^{\prime}} g_{0}^{\gamma_{0}^{\prime}} g_{1}^{\gamma_{1}^{\prime}} \cdots g_{k}^{\gamma_{k}^{\prime}}\right)$ has corner point at the key point on the boundary of $\mathrm{NP}_{k}(f),\left(\alpha_{k}, \beta_{k}\right)$ with

$$
\begin{aligned}
\alpha_{k} & =s_{k, l_{k}} p_{k} \cdots p_{h}-\tau_{k}, \\
\beta_{k} & =p_{k} \cdots p_{h}-p_{k}
\end{aligned}
$$

where $q_{k}=s_{k, l_{k}} p_{k}-\tau_{k}$. 
Proof of Theorem 1.1. The proof is the same for $k=1$ as

$$
\psi_{1}=q_{1} p_{1} \cdots p_{h}
$$

and

$$
\delta_{0}=p_{1} \cdots p_{h}
$$

so that

$$
\psi_{1} \in \text { semi-group }\left\{\delta_{0}\right\} .
$$

Under our new assumptions

$$
\gamma_{k}^{\prime}=p_{k} \cdots p_{h}-p_{k}
$$

and

$$
p_{1} \cdots p_{k-1} \gamma_{0}^{\prime}+q_{1} p_{2} \cdots p_{k-1} \gamma_{1}^{\prime}+\cdots+q_{k-1} \gamma_{k-1}^{\prime}+s_{k, l_{k}} \gamma_{k}^{\prime}=s_{k, l_{k}} p_{k} \cdots p_{h}-\tau_{k} .
$$

Therefore

$p_{1} \cdots p_{k-1} \gamma_{0}^{\prime}+q_{1} p_{2} \cdots p_{k-1} \gamma_{1}^{\prime}+\cdots+q_{k-1} \gamma_{k-1}^{\prime}+s_{k, l_{k}}\left(p_{k} \cdots p_{h}-p_{k}\right)=s_{k, l_{k}} p_{k} \cdots p_{h}-\tau_{k}$

and thus

$$
p_{1} \cdots p_{k-1} \gamma_{0}^{\prime}+q_{1} p_{2} \cdots p_{k-1} \gamma_{1}^{\prime}+\cdots+q_{k-1} \gamma_{k-1}^{\prime}=s_{k, l_{k}} p_{k}-\tau_{k}=q_{k} .
$$

Multiply each side by $p_{k} \cdots p_{h}$ to obtain

$$
\delta_{0} \gamma_{0}^{\prime}+\delta_{1} \gamma_{1}^{\prime} \cdots \delta_{k-1} \gamma_{k-1}^{\prime}=\psi_{k} .
$$

As $\gamma_{i}^{\prime} \geq 0$, for all $i$, the result follows and

$$
\psi_{k} \in \text { semi-group }\left\{\delta_{0}, \ldots, \delta_{k-1}\right\} .
$$

\section{The semi-group conditions for one point at infinity}

In section 3 the main assumption underlying the proof of the Abhyankar-Moh semigroup theorem (Theorem 1.1) is that, for $f \in \mathbb{C}[x, y]$, the curve defined by with $f(x, y)=0$ is an irreducible algebraic curve in the complex plane $\mathbb{C}^{2}$ with "one place at infinity." Alternatively the minimal link at infinity is of the following form

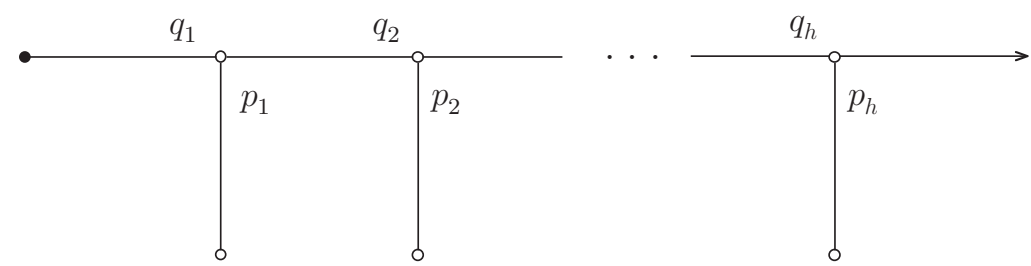


There are two key consequences of this assumption that are used to prove Theorem 1.1:

(i) $f$ is monic in $y$ so that we can find approximate roots of $f$, and

(ii) the Newton polygons of $f$ each have one key line segment and give just one Newton pair corresponding to one branch in the splice diagram.

This leads to the intriguing question: can we find descriptions of the linking numbers for curves in other cases? The answer is that we can indeed find partial descriptions in two cases. In particular, in this section we give a description for the case of one point at infinity, but more than one branch at the $h^{\text {th }}$ node.

In this situation, at some node, say, the $h^{\text {th }}$ node the cabling operation consists of adding $l$ parallel $\left(p_{h}, q_{h}\right)$ cables, $l \geq 1$. The splice diagram is

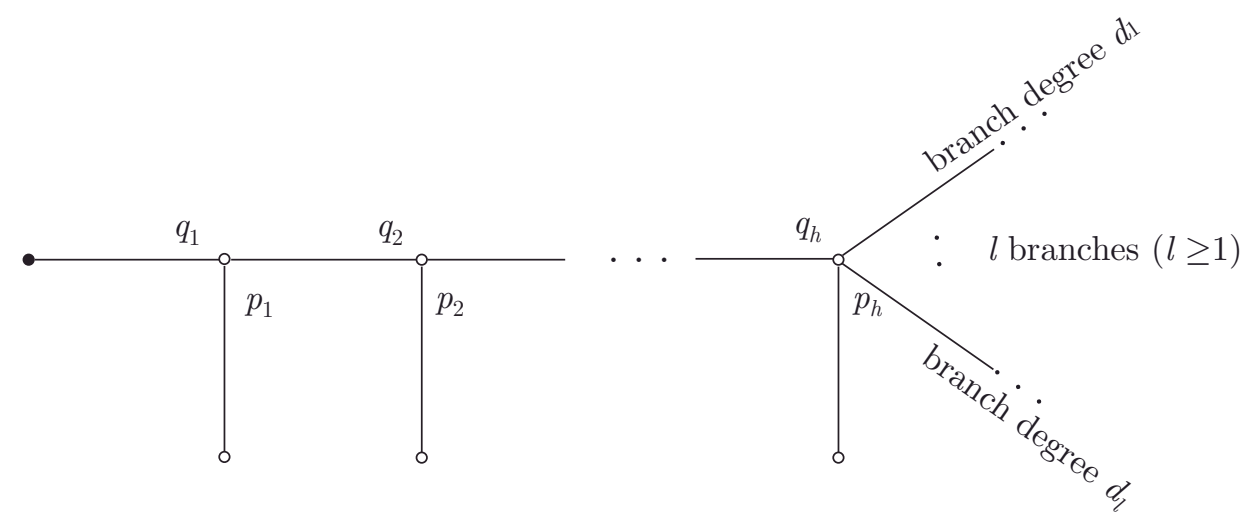

either with $l=1$ and $p_{1}, \ldots, p_{h} \geq 2$ or with $l \geq 2, p_{1}, \ldots, p_{h-1} \geq 2$, and $p_{h} \geq 1$. The above splice diagram is minimal except unless $l \geq 2$ and $p_{h}=1$.

Note that the branch degree is found as follows. Let $v$ be a node in $\Gamma$ and select a number of edges adjoining $v$. Let $w_{1}, \ldots, w_{r}$ be the arrowheads in that part of the splice diagram which lie along and beyond the selected edges. Then the branch degree is $\sum_{i=1}^{r} l\left(v, w_{i}\right)$ where $l\left(v, w_{i}\right)$ is the product of the edge weights adjacent to but not on the path from $v$ to $w_{i}$.

Let $\sum_{i=1}^{l} d_{i}=d$. Name the vertices in the diagram above as follows. Starting from the root vertex and moving towards the branching vertex:

- vertices with one adjoining edge are $u_{0}, u_{1}, \ldots, u_{h}$

- vertices with three or more adjoining edges are $v_{1}, v_{2}, \ldots, v_{h}$

Let $S_{u_{i}}$ and $S_{v_{i}}$ denote the virtual components of the link corresponding to the nodes $\left\{u_{i}\right\}_{i=0}^{h}$ and $\left\{v_{j}\right\}_{j=1}^{h}$ respectively. Then name the linking numbers of the virtual 
components with $L$, the link at infinity, by

$$
\begin{gathered}
\delta_{0}=l_{u_{0}}=\operatorname{link}\left(S_{u_{0}}, L\right)=p_{1} p_{2} \cdots p_{h} d, \\
\delta_{1}=l_{u_{1}}=\operatorname{link}\left(S_{u_{1}}, L\right)=q_{1} p_{2} p_{3} \cdots p_{h} d, \\
\delta_{2}=l_{u_{2}}=\operatorname{link}\left(S_{u_{2}}, L\right)=q_{2} p_{3} p_{4} \cdots p_{h} d, \\
\vdots \\
\delta_{h}=l_{u_{h}}=\operatorname{link}\left(S_{u_{h}}, L\right)=q_{h} d,
\end{gathered}
$$

and

$$
\begin{gathered}
\psi_{1}=l_{v_{1}}=\operatorname{link}\left(S_{v_{1}}, L\right)=q_{1} p_{1} p_{2} \cdots p_{h} d, \\
\psi_{2}=l_{v_{2}}=\operatorname{link}\left(S_{v_{2}}, L\right)=q_{2} p_{2} p_{3} \cdots p_{h} d, \\
\vdots \\
\psi_{h}=l_{v_{h}}=\operatorname{link}\left(S_{v_{h}}, L\right)=q_{h} p_{h} d .
\end{gathered}
$$

Theorem 1.2. Let $f \in \mathbb{C}[x, y]$ be an irreducible polynomial that defines a curve $f(x, y)=0$ with one point at infinity. Then, using the above notation we have

(i) For $i=1, \ldots, h-1$,

$$
\psi_{i} \in \operatorname{semi-group}\left\{\delta_{0}, \delta_{1}, \ldots, \delta_{i-1}\right\}
$$

and

(ii) there exists $r \in \mathbb{Z}$ such that $1 \leq r \leq l$ such that

$$
r \psi_{h} \in \operatorname{semi-group}\left\{\delta_{0}, \delta_{1}, \ldots, \delta_{h-1}\right\} .
$$

The following lemma is used in the proof of this theorem.

Lemma 4.1. Let $w \in \mathbb{C}[z]$ have exactly $l$ distinct roots, with $l>1$ and assume $w(z)=z^{n}+a_{n-1} z^{n-1}+\cdots+a_{0}$. Then at least one of $a_{n-1}, a_{n-2}, \ldots, a_{n-l} \neq 0$.

Proof. Assume that

$$
w(z)=\prod_{i=1}^{n}\left(z-b_{i}\right)=\prod_{j=1}^{l}\left(z-c_{j}\right)^{d_{j}}
$$

where $b_{1}, b_{2}, \ldots, b_{n}$ are the roots of $w$ repeated according to multiplicity and $c_{1}, \ldots$, $c_{l}$ are the $l$ distinct roots. We prove the result by contradiction. Assume

$$
a_{n-1}=a_{n-2}=\cdots=a_{n-l}=0 .
$$

Now $a_{n-1}=-\sigma_{1}, \ldots, a_{n-l}=(-1)^{l} \sigma_{l}$ where $\sigma_{t}$ is the $t^{\text {th }}$ symmetric function in $b_{1}$, $\ldots, b_{n}$,

$$
\sigma_{t}=\sum_{i_{1}<i_{2}<\cdots<i_{t}} b_{i_{1}} b_{i_{2}} \cdots b_{i_{t}} .
$$


Let

$$
S_{t}=\sum_{i=1}^{n} b_{i}^{t}
$$

Then $\sigma_{t}$ and $S_{t}$ are linked by Newton's formulae as follows:

$$
S_{t}-\sigma_{1} S_{t-1}+\sigma_{2} S_{t-2}-\cdots+(-1)^{t-1} \sigma_{t-1} S_{1}+(-1)^{t} S_{0} \sigma_{t}=0
$$

for $t=1,2, \ldots$ Given $S_{0}=t \neq 0$ for $t \geq 1$,

$$
\sigma_{1}=\cdots=\sigma_{l} \quad \text { if and only if } S_{1}=\cdots=S_{l}=0 .
$$

Now $S_{t}=\sum_{i=1}^{n} b_{i}^{t}=\sum_{j=1}^{l} d_{j} c_{j}^{t}$ so that as $\sigma_{t}=0$ for $t=1,2, \ldots, l$,

$$
\sum_{j=1}^{l} d_{j} c_{j}^{t}=0 \quad \text { for } t=1, \ldots, l .
$$

These equations are linear in $d_{j}$. Therefore

$$
\left[\begin{array}{cccc}
c_{1} & c_{2} & \cdots & c_{l} \\
c_{1}^{2} & c_{2}^{2} & \cdots & c_{l}^{2} \\
\vdots & & & \vdots \\
c_{1}^{l} & c_{2}^{l} & \cdots & c_{l}^{l}
\end{array}\right]\left[\begin{array}{c}
d_{1} \\
d_{2} \\
\vdots \\
d_{l}
\end{array}\right]=\left[\begin{array}{c}
0 \\
0 \\
\vdots \\
0
\end{array}\right] .
$$

As none of the $d_{j}=0$ for $j=1, \ldots, l$, the coefficient matrix has zero determinant. Hence

$$
\begin{aligned}
\left|\begin{array}{cccc}
c_{1} & c_{2} & \cdots & c_{l} \\
c_{1}^{2} & c_{2}^{2} & \cdots & c_{l}^{2} \\
\vdots & & & \vdots \\
c_{1}^{l} & c_{2}^{l} & \cdots & c_{l}^{l}
\end{array}\right| & =c_{1} c_{2} \cdots c_{l}\left|\begin{array}{cccc}
1 & 1 & \cdots & 1 \\
c_{1} & c_{2} & \cdots & c_{l} \\
\vdots & & & \vdots \\
c_{1}^{l-1} & c_{2}^{l-1} & \cdots & c_{l}^{l-1}
\end{array}\right| \\
& =c_{1} c_{2} \cdots c_{l} \cdot(\text { Vandermonde determinant }) \\
& =c_{1} c_{2} \cdots c_{l} \prod_{i<j}\left(c_{i}-c_{j}\right) \\
& =0 .
\end{aligned}
$$

As we have exactly $l$ distinct factors, $c_{i} \neq c_{j}$ for $i \neq j$ we must have $c_{j}=0$ for some $j$. Without loss of generality assume $c_{l}=0$.

Divide $w$ by $z^{n_{l}}$ to obtain $\bar{w}$. Then

$$
\bar{w}(z)=z^{n-l}+a_{n-1} z^{n-l-1}+\cdots+a_{l}=\pi_{j=1}^{l-1}\left(z-c_{j}\right)^{n_{j}}
$$

with $a_{n-1}=\cdots=a_{n-l+1}=0$. By the same reasoning as above we get $c_{j}=0$ for some $j<l$. But then $c_{j}=c_{l}$ which contradicts our assumption that we have exactly $l$ factors. 
Proof of Theorem 1.2. The proof of (i) is identical to that of Theorem 1.1 because of the following:

(i) As $f$ is monic in $y$ we can find the first $h-1$ approximate roots of $f, g_{0}, g_{1}$, $\ldots, g_{h-1}$, as before. That is, as $f \in \mathbb{C}[x, y]$ is monic in $y$ and $\operatorname{deg}_{y}(f)=$ $p_{1} p_{2} \cdots p_{k} \cdots p_{h} d$ we put $g_{0}=x$ and for $k=1,2, \ldots, h-1$ there exists a unique polynomial $g_{k}$ (monic in $y$ ) such that

$$
f=g_{k}^{p_{k} \cdots p_{h} d}+\zeta_{k}
$$

where $\operatorname{deg}_{y}\left(\zeta_{k}\right)<p_{1} p_{2} \cdots p_{k-1}$. Here $g_{k}$ is the $\left(p_{k} \cdots p_{h} d\right)^{\text {th }}$ approximate root of $f$. Then

$$
\zeta_{k}=\sum_{\gamma_{0} \ldots \gamma_{k}} a_{\gamma_{0} \ldots \gamma_{k}} g_{0}^{\gamma_{0}} g_{1}^{\gamma_{1}} \cdots g_{k}^{\gamma_{k}}
$$

where $g_{0}=x$ and $g_{1}, g_{2}, \ldots, g_{k-1}$ are the $\left(p_{1} p_{2} \cdots p_{h} d\right)^{\text {th }},\left(p_{2} \cdots p_{h} d\right)^{\text {th }}, \ldots$, $\left(p_{k-1} \cdots p_{h} d\right)^{\text {th }}$ approximate roots of $f$ respectively. In addition, $0 \leq \gamma_{0}, 0 \leq$ $\gamma_{1}<p_{1}, \ldots, 0 \leq \gamma_{k-1}<p_{k-1}, 0 \leq \gamma_{k}<p_{k} \cdots p_{h} d-1$.

(ii) No two of the corner points of $\mathrm{NP}_{k}\left(g_{k}^{p_{k} \cdots p_{h} d}\right)$ and $\mathrm{NP}_{k}\left(a_{\gamma_{0} \ldots \gamma_{k}} g_{0}^{\gamma_{0}} g_{1}^{\gamma_{1}} \cdots g_{k}^{\gamma_{k}}\right)$ coincide.

(iii) At each of the first $h-1$ stages of finding the cabling pairs for the component of the link at infinity at $y=0$, there just one key line segment in $\mathrm{NP}_{k}(f)$ that gives just one cabling pair.

Given this we see that all the results of the technical lemmas in section 3.5 and the corresponding results in section 3.6 will carry over for $f$.

To prove (ii) we first note that if $l=1$ then Theorem 1.2 is exactly Theorem 1.1. So assume that $l \geq 2$. We start with the case for no redundant cables at the $h^{\text {th }}$ stage. Consider the Newton polygon at stage $h$. For $p_{h} \geq 2$ it is of the form

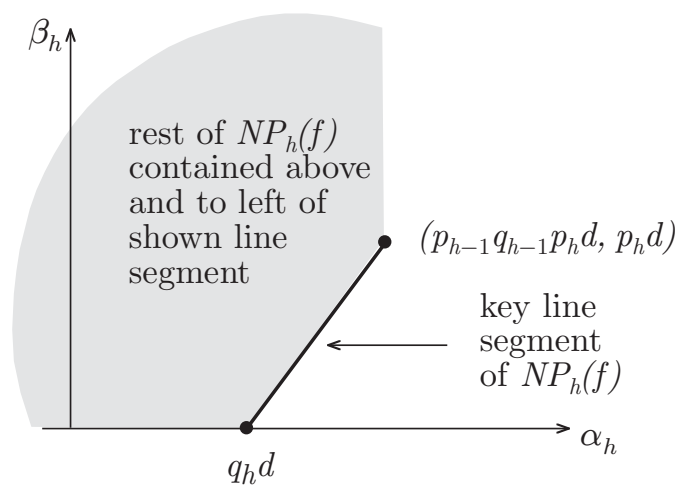


However it is possible that for $p_{h}=1$ there may be more than one line segment corresponding to the branches at this node. The boundary of $\mathrm{NP}_{h}(f)$ will still include that part of the line segment marked on the following diagram:

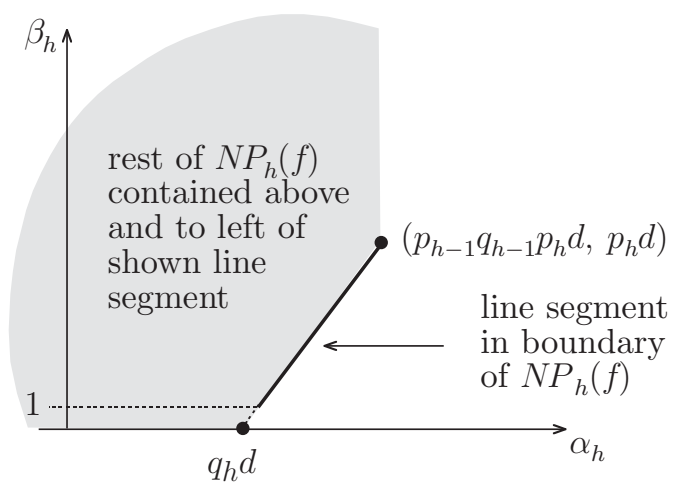

Therefore for $p_{h} \geq 1$ there is a line segment of slope $p_{h} / \tau_{h}$. Thus we can express $f_{h}$ in the form

$$
f_{h}=\prod_{i=1}^{l} b_{h}\left(y_{h}^{p_{h}}-t_{h, i}^{p_{h}} x_{h}^{-\tau_{h}}\right)^{d_{i}}+\sum_{\alpha_{h} p_{h}-\beta_{h} q_{h}<q_{h} d} b_{\alpha_{h} \beta_{h}} x_{h}^{\alpha_{h}} y_{h}^{\beta_{h}}
$$

for some $b_{h} \neq 0, b_{\alpha_{h} \beta_{h}} \in \mathbb{C}$, where $t_{h, i}^{p_{h}} \neq t_{h, j}^{p_{h}}$ for $i \neq j$. Consider the expansion of the terms in $f_{h}$ corresponding to the line segment of slope $p_{h} / \tau_{h}$ :

$$
\prod_{i=1}^{l} b_{h}\left(y_{h}^{p_{h}}-t_{h, i}^{p_{h}} x_{h}^{-\tau_{h}}\right)^{d_{i}}=b_{h} \sum_{r=0}^{d} c_{r} x_{h}^{q_{h-1} p_{h-1} p_{h} d-r \tau_{h}} y_{h}^{p_{h} d-r p_{h}} .
$$

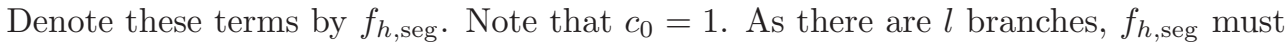
have exactly $l$ distinct factors. Thus from Lemma 4.1, one of $c_{1} \cdots c_{l} \neq 0$. Thus there must be at least one key point in $f_{h, \text { seg }}$ for $r=1,2, \ldots, l$. Following the same reasoning as we used in Lemma 3.26, we conclude that there exists some $a_{\gamma_{0}^{\prime} \ldots \gamma_{h}^{\prime}} g_{0}^{\gamma_{0}^{\prime}} \cdots g_{h}^{\gamma_{h}^{\prime}}$ with non-zero coefficient such that

$$
\begin{aligned}
\left(p_{1} \cdots p_{h-1} \gamma_{0}^{\prime}+q_{1} p_{2} \cdots p_{h-1} \gamma_{1}^{\prime}+\cdots+q_{h-1} \gamma_{h-1}^{\prime}+q_{h-1} p_{h-1} \gamma_{h}^{\prime}, \gamma_{h}^{\prime}\right) & \\
= & \left(q_{h-1} p_{h-1} p_{h} d-r \tau_{h}, p_{h} d-r p_{h}\right)
\end{aligned}
$$

for some $r \in\{1,2, \ldots, l\}$. As $\gamma_{h}^{\prime}=p_{h} d-r p_{h}$,

$$
\begin{array}{r}
p_{1} \cdots p_{h-1} \gamma_{0}^{\prime}+q_{1} p_{2} \cdots p_{h-1} \gamma_{1}^{\prime}+\cdots+q_{h-1} \gamma_{h-1}^{\prime}+q_{h-1} p_{h-1}\left(p_{h} d-r p_{h}\right) \\
=q_{h-1} p_{h-1} p_{h} d-r \tau_{h}
\end{array}
$$


Hence

$$
p_{1} \cdots p_{h-1} \gamma_{0}^{\prime}+q_{1} p_{2} \cdots p_{h-1} \gamma_{1}^{\prime}+\cdots+q_{h-1} \gamma_{h-1}^{\prime}=q_{h-1} p_{h-1} r p_{h}-r \tau_{h}=r q_{h} .
$$

Multiplying both sides by $p_{h} d$ gives the required result.

The argument for the case of redundant cables is the similar to that used in section 3.6 except that we obtain

$$
\gamma_{h}^{\prime}=p_{h} d-r p_{h}
$$

and

$$
p_{1} \cdots p_{h-1} \gamma_{0}^{\prime}+q_{1} p_{2} \cdots p_{h-1} \gamma_{1}^{\prime}+\cdots+q_{h-1} \gamma_{h-1}^{\prime}+s_{h, l_{h}} \gamma_{h}^{\prime}=s_{h, l_{h}} p_{h} d-r \tau_{h}
$$

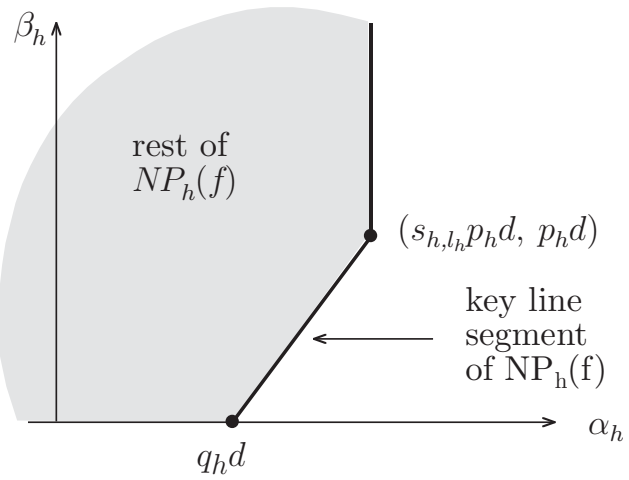

Therefore

$$
\begin{array}{r}
p_{1} \cdots p_{h-1} \gamma_{0}^{\prime}+q_{1} p_{2} \cdots p_{h-1} \gamma_{1}^{\prime}+\cdots+q_{h-1} \gamma_{h-1}^{\prime}+s_{h, l_{h}}\left(p_{h} d-r p_{h}\right) \\
=s_{h, l_{h}} p_{h} d-r \tau_{k}
\end{array}
$$

and

$$
p_{1} \cdots p_{h-1} \gamma_{0}^{\prime}+q_{1} p_{2} \cdots p_{h-1} \gamma_{1}^{\prime}+\cdots+q_{h-1} \gamma_{k-1}^{\prime}=s_{h, l_{h}} r p_{h}-r \tau_{h}=r q_{h} .
$$

Multiply each side by $p_{k} \cdots p_{h}$ to obtain

$$
\delta_{0} \gamma_{0}^{\prime}+\delta_{1} \gamma_{1}^{\prime} \cdots \delta_{h-1} \gamma_{h-1}^{\prime}=r \psi_{k}
$$

As $\gamma_{i}^{\prime} \geq 0$, for all $i$, the result follows and

$$
r \psi_{h} \in \text { semi-group }\left\{\delta_{0}, \ldots, \delta_{k-1}\right\}
$$

for some $r \in\{1,2, \ldots, l\}$. 


\section{The case with more points at infinity}

Theorem 5.1. Assume Let $f \in \mathbb{C}[x, y]$ be an irreducible polynomial that defines a curve $f(x, y)=0$ satisfying the following conditions:

- The splice diagram is

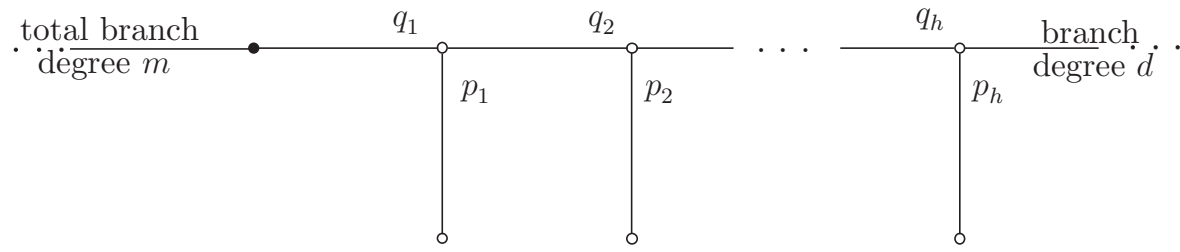

so that the polynomial has either one $(m=0)$ or two $(m>0)$ points at infinity.

- the section shown with cabling pairs $\left(p_{1}, q_{1}\right), \ldots,\left(p_{h}, q_{h}\right)$ occurs on the branch corresponding to the point at infinity $y=0$,

- $f=\rho+x^{m} \xi$ with $\rho, \xi \in \mathbb{C}[x, y]$ so that $x^{-m} f \in \mathbb{C}\left[x^{-1}, x, y\right]$ is monic in $y$,

- $\operatorname{deg}_{y}(f)=\operatorname{deg}_{y}(\xi)=p_{1} p_{2} \cdots p_{h} d$ for some $d \in \mathbb{C}$ and

- $\operatorname{deg}_{y}(\rho)<\operatorname{deg}_{y}(\xi)-p_{1} p_{2} \cdots p_{h-1}=p_{1} p_{2} \cdots p_{h} d-p_{1} p_{2} \cdots p_{h-1}, \operatorname{deg}_{x}(\rho)<m$.

Then, for $j=1,2, \ldots, h$,

$$
\begin{aligned}
m p_{1} p_{2} \cdots p_{h} d+\psi_{j} \in \operatorname{semi-group}\left\{p_{1} p_{2} \cdots p_{h} d, q_{1} p_{2} p_{3} \cdots p_{h} d,\right. & \\
& \left.\ldots, q_{j-1} p_{j} p_{j+1} \cdots p_{h} d\right\} \cup\{0\}
\end{aligned}
$$

The Abhyankar-Moh semi-group conditions are the case $m=0$ and $d=1$.

The condition on the $y$-degree of $\rho$ ensures, for $g_{k}$ the $\left(p_{k} \cdots p_{h}\right)^{\text {th }}$ root of $x^{-m} f$, that $x^{m} g_{k}^{p_{k} \cdots p_{h} d}$ is a polynomial and that $\mathrm{NP}\left(x^{m} g_{k}^{p_{k} \cdots p_{h} d}\right)$ lies inside $\mathrm{NP}(f)$ for $k=$ $1, \ldots, h$. It is in fact sufficient that $\zeta_{k}=f-x^{m} g_{k}^{p_{k} \cdots p_{h} d}$ is such that $\operatorname{deg}_{y}\left(\zeta_{k}\right)<$ $p_{1} \cdots p_{h} d-p_{1} \cdots p_{k-1}$ for all $k$. Given the above we have $\operatorname{deg}_{x}(f)=q_{1} p_{2} \cdots p_{h} d+m$ and $q_{1}<p_{1}$, and that $\mathrm{NP}(f)$ is as follows: 


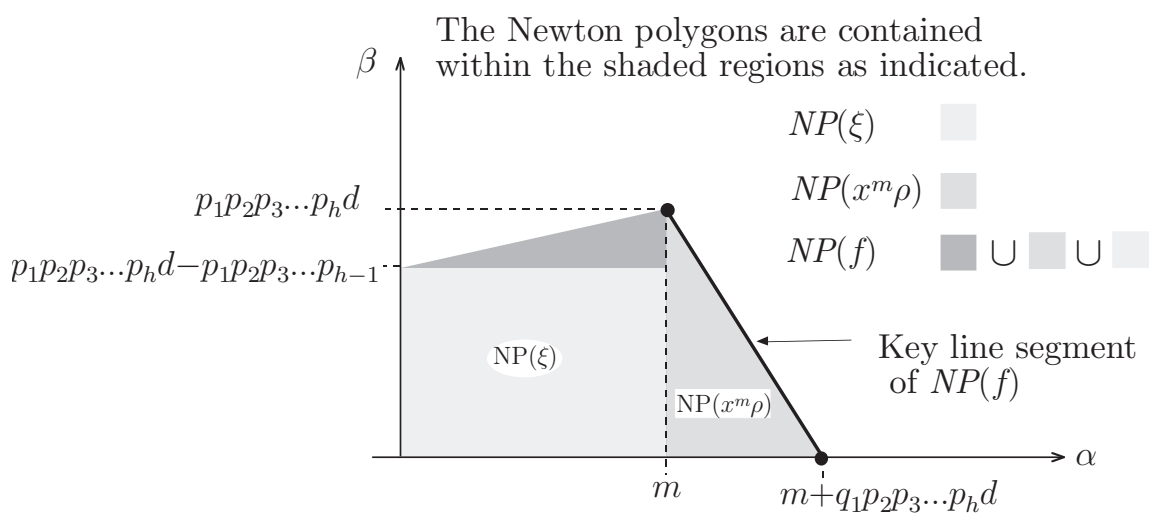

The link at infinity has two parts, that part that is found at $y=0$ and that at $x=0$. The branch under consideration corresponds to $y=0$. Let $L_{y}$ be the union of the components of the link at infinity at $y=0$. We then name the vertices in the diagram corresponding to the branch $y=0$ in the same way as in section 4 . We again let $S_{u_{i}}$ and $S_{v_{i}}$ denote the virtual components of the link corresponding to the nodes $\left\{u_{i}\right\}_{i=0}^{h}$ and $\left\{v_{j}\right\}_{j=1}^{h}$ respectively. We name the linking numbers of the virtual components with $L_{y}$ by

$$
\begin{aligned}
\delta_{0}=l_{u_{0}} & =\operatorname{link}\left(S_{u_{0}}, L_{y}\right)=p_{1} p_{2} \cdots p_{h} d \\
\delta_{1}=l_{u_{1}} & =\operatorname{link}\left(S_{u_{1}}, L_{y}\right)=q_{1} p_{2} p_{3} \cdots p_{h} d \\
\delta_{2}=l_{u_{2}} & =\operatorname{link}\left(S_{u_{2}}, L_{y}\right)=q_{2} p_{3} p_{4} \cdots p_{h} d \\
& \vdots \\
\delta_{h}=l_{u_{h}} & =\operatorname{link}\left(S_{u_{h}}, L_{y}\right)=q_{h} d
\end{aligned}
$$

and

$$
\begin{gathered}
\psi_{1}=l_{v_{1}}=\operatorname{link}\left(S_{v_{1}}, L_{y}\right)=q_{1} p_{1} p_{2} \cdots p_{h} d \\
\psi_{2}=l_{v_{2}}=\operatorname{link}\left(S_{v_{2}}, L_{y}\right)=q_{2} p_{2} p_{3} \cdots p_{h} d \\
\vdots \\
\psi_{h}=l_{v_{h}}=\operatorname{link}\left(S_{v_{h}}, L_{y}\right)=q_{h} p_{h} d
\end{gathered}
$$

If $m \geq 1$, the curve has two points at infinity and the link at infinity has two or more components. The points at infinity for a curve $f(x, y)=0$ are the points of intersection of the closure of $f(x, y)=0$ in $\mathbb{C P}^{2}$ with the line at infinity $\mathbb{C P}^{1}$.

In the case that $q_{k-1} \leq 0$ so that $q_{k}<0$, the above semi-group conditions do not restrict the value of $q_{k}$ in any further way.

Let $g=x^{-m} f=x^{-m} \rho+\xi$. We note the following: 
(i) As $g$ is monic in $y$ we can find approximate roots of $g, g_{0}, g_{1}, \ldots, g_{h}$, as before. The condition $\operatorname{deg}_{y}(\rho)<p_{1} p_{2} \cdots p_{h} d-p_{1} p_{2} \cdots p_{h-1}$ means that $g_{1}, \ldots, g_{h}$ are the approximate roots of $\xi$ so that $x^{m} g_{k}^{p_{k} \cdots p_{h} d}, k=1, \ldots, h$ are all polynomials. Then

$$
g=g_{k}^{p_{k} \cdots p_{h} d}+\zeta_{k}
$$

where $\zeta_{k} \in \mathbb{C}\left[x, x^{-1}, y\right]$ with $\operatorname{deg}_{x}\left(\zeta_{k}\right) \geq-m$ and $\operatorname{deg}_{y}\left(\zeta_{k}\right)<p_{1} \cdots p_{h} d-$ $p_{1} \cdots p_{k-1}$. As before

$$
\zeta_{k}=\sum a_{\gamma_{0} \ldots \gamma_{k}} g_{0}^{\gamma_{0}} \cdots g_{k}^{\gamma_{k}}
$$

where $0 \leq \gamma_{1}<p_{1}, \ldots, 0 \leq \gamma_{k-1}<p_{k-1}, 0 \leq \gamma_{k}<p_{k} \cdots p_{h} d-1$. However, $\gamma_{0}$ is no longer a non-negative integer. It now satisfies the condition $\gamma_{0} \geq-m$.

(ii) The uniqueness of the corner points of the terms of $g$ still holds as the Lemmas 3.22 and 3.23 did not depend upon $\gamma_{0}$ and $\gamma_{0}^{\prime}$ or any $q_{i}$ being positive.

(iii) At each of the first $h$ stages of finding the cabling pairs for the component of link at infinity at $y=0$, there is a key line segment in $\operatorname{NP}_{k}(g)$ and this gives just one branch in the splice diagram.

Given this we see that all the results of the technical lemmas in section 3.5 and the corresponding results in section 3.6 will carry over for $g$.

As the $k^{\text {th }}$ Newton polygon of $x$ is a point on the $\alpha_{k}$-axis, the effect of multiplying $g$ by $x^{m}$ is to translate each Newton polygon to the right by a multiple of $m$. In addition, we see that the equations used to find the first $h$ Newton pairs of $f$ and $g$ will be the same except of a factor $x^{c_{k} m}$ for some constant $c_{k}$ at each stage. Thus the first $h$ Newton pairs of $f$ and $g$ will be identical.

Proof of Theorem 5.1. Using the same logic as in the proof of Theorem 1.1 we again find that the result is clear for $k=1$ as

$$
\psi_{1}=q_{1} p_{1} \cdots p_{h} d
$$

and

$$
\delta_{0}=p_{1} \cdots p_{h} d
$$

so that $q_{1} \geq 0$

$$
m p_{1} \cdots p_{h} d+\psi_{1} \in \operatorname{semi-group}\left\{\delta_{0}\right\} \cup\{0\} .
$$

From the above discussion we note that the assumptions used in the proof of Theorem 1.1 hold for $g$. Where there are no redundant Newton pairs these are:

(i) that the terms of $g$ with non-zero coefficients have Newton polygons with corner points occurring at $\left(\alpha_{k}, \beta_{k}\right)$ where

$$
\begin{aligned}
\alpha_{k} & =p_{1} \cdots p_{k-1} \gamma_{0}+q_{1} p_{2} \cdots p_{k-1} \gamma_{1}+\cdots+q_{k-1} \gamma_{k-1}+q_{k-1} p_{k-1} \gamma_{k}, \\
\beta_{k} & =\gamma_{k},
\end{aligned}
$$


(ii) that there is some term in $g$

$$
a_{\gamma_{0}^{\prime} \ldots \gamma_{k}^{\prime}} g_{0}^{\gamma_{0}^{\prime}} \cdots g_{k}^{\gamma_{k}^{\prime}}
$$

$a_{\gamma_{0}^{\prime} \ldots \gamma_{k}^{\prime}} \neq 0$ whose Newton polygon $\mathrm{NP}_{k}\left(a_{\gamma_{0}^{\prime} \ldots \gamma_{k}^{\prime}} g_{0}^{\gamma_{0}^{\prime}} g_{1}^{\gamma_{1}^{\prime}} \cdots g_{k}^{\gamma_{k}^{\prime}}\right)$ has corner point at the key point on the boundary of $\mathrm{NP}_{k}(g),\left(\alpha_{k}, \beta_{k}\right)$ with

$$
\begin{aligned}
\alpha_{k} & =q_{k-1} p_{k-1} p_{k} \cdots p_{h} d-\tau_{k}, \\
\beta_{k} & =p_{k} \cdots p_{h} d-p_{k},
\end{aligned}
$$

where $q_{k}=q_{k-1} p_{k-1} p_{k}-\tau_{k}$.

We then have

$$
\gamma_{k}^{\prime}=p_{k} \cdots p_{h} d-p_{k}
$$

and

$$
\begin{aligned}
p_{1} \cdots p_{k-1} \gamma_{0}^{\prime}+q_{1} p_{2} \cdots p_{k-1} \gamma_{1}^{\prime}+\cdots+q_{k-1} \gamma_{k-1}^{\prime}+q_{k-1} p_{k-1} \gamma_{k}^{\prime} & \\
& =q_{k-1} p_{k-1} p_{k} \cdots p_{h} d-\tau_{k} .
\end{aligned}
$$

Therefore

$$
\begin{array}{r}
p_{1} \cdots p_{k-1} \gamma_{0}^{\prime}+q_{1} p_{2} \cdots p_{k-1} \gamma_{1}^{\prime}+\cdots+q_{k-1} \gamma_{k-1}^{\prime}+q_{k-1} p_{k-1}\left(p_{k} \cdots p_{h} d-p_{k}\right) \\
=q_{k-1} p_{k-1} p_{k} \cdots p_{h} d-\tau_{k}
\end{array}
$$

and thus

$$
p_{1} \cdots p_{k-1} \gamma_{0}^{\prime}+q_{1} p_{2} \cdots p_{k-1} \gamma_{1}^{\prime}+\cdots+q_{k-1} \gamma_{k-1}^{\prime}=q_{k-1} p_{k-1} p_{k}-\tau_{k}=q_{k} .
$$

Multiply each side by $p_{k} \cdots p_{h} d$ to obtain

$$
\delta_{0} \gamma_{0}^{\prime}+\delta_{1} \gamma_{1}^{\prime} \cdots \delta_{k-1} \gamma_{k-1}^{\prime}=\psi_{k}
$$

We have $\gamma_{i}^{\prime} \geq 0$, for $i=1, \ldots, k$ and $\gamma_{0} \geq-m$. To ensure that $\delta_{0}$ has a non-negative coefficient add $m \delta_{0}$ to each side to obtain

$$
\delta_{0}\left(m+\gamma_{0}^{\prime}\right)+\delta_{1} \gamma_{1}^{\prime} \cdots \delta_{k-1} \gamma_{k-1}^{\prime}=\delta_{0} m+\psi_{k}
$$

which is the required result as $\delta_{0}=p_{1} \cdots p_{h} d$. Note, it is possible that $\operatorname{gcd}\left(p_{k}, q_{k}\right)=1$ while $\operatorname{gcd}\left(p_{k}, m p_{1} \cdots p_{k-1}+q_{k}\right) \neq 1$ so that we may have $m p_{1} \cdots p_{k-1}+q_{k}=0$. Thus

$$
m p_{1} \cdots p_{h} d+\psi_{k} \in \text { semi-group }\left\{\delta_{0}, \ldots, \delta_{k-1}\right\} \cup\{0\} .
$$

To prove Theorem 5.1 in the case where there are redundant Newton pairs one can use the same techniques as in section 3.6. 
Acknowledgement. I wish to thank Walter Neumann for introducing me to an exciting and stimulating area of mathematics and for his help and encouragement.

\section{References}

[1] S. S. Abhyankar, Lectures on expansion techniques in algebraic geometry, with notes by B. Singh, Tata Institute of Fundamental Research Lectures on Mathematics and Physics, vol. 57, Tata Institute of Fundamental Research, Bombay, 1977.

[2] _ Algebraic geometry for scientists and engineers, Mathematical Surveys and Monographs, vol. 35, American Mathematical Society, Providence, RI, 1990.

[3] S. S. Abhyankar and T. T. Moh, Newton-Puiseux expansion and generalized Tschirnhausen transformation. I, J. Reine Angew. Math. 260 (1973), 47-83.

[4] _ Embeddings of the line in the plane, J. Reine Angew. Math. 276 (1975), 148-166.

[5] N. A'Campo and M. Oka, Geometry of plane curves via Tschirnhausen resolution tower, Osaka J. Math. 33 (1996), no. 4, 1003-1033.

[6] E. Artal-Bartolo, Une démonstration géométrique du théorème d'Abhyankar-Moh, J. Reine Angew. Math. 464 (1995), 97-108.

[7] E. Artal-Bartolo, P. Cassou-Noguès, and A. Dimca, Sur la topologie des polynômes complexes, Singularities (Oberwolfach, 1996), Progr. Math., vol. 162, Birkhäuser, Basel, 1998, pp. 317-343.

[8] E. Artal-Bartolo, P. Cassou-Noguès, and I. Luengo Velasco, On polynomials whose fibers are irreducible with no critical points, Math. Ann. 299 (1994), no. 3, 477-490.

[9] E. Brieskorn and H. Knörrer, Plane algebraic curves, translated by J. Stillwell, Birkhäuser Verlag, Basel, 1986.

[10] P. Cassou-Noguès, Entrelacs toriques itérés et intégrales associées à une courbe plane, Sém. Théor. Nombres Bordeaux (2) 2 (1990), no. 2, 273-331.

[11] $\ldots$, The effect of rational maps on polynomial maps, Ann. Polon. Math. 76 (2001), no. 1-2, $21-31$.

[12] D. Eisenbud and W. Neumann, Three-dimensional link theory and invariants of plane curve singularities, Annals of Mathematics Studies, vol. 110, Princeton University Press, Princeton, NJ, 1985.

[13] F. Kirwan, Complex algebraic curves, London Mathematical Society Student Texts, vol. 23, Cambridge University Press, Cambridge, 1992.

[14] S. Lefschetz, Algebraic geometry, Princeton University Press, Princeton, N. J., 1953.

[15] J. Milnor, Singular points of complex hypersurfaces, Annals of Mathematics Studies, No. 61, Princeton University Press, Princeton, N.J., 1968.

[16] M. Miyanishi, Minimization of the embeddings of the curves into the affine plane, J. Math. Kyoto Univ. 36 (1996), no. 2, 311-329.

[17] Y. Nakazawa and M. Oka, Smooth plane curves with one place at infinity, J. Math. Soc. Japan 49 (1997), no. 4, 663-687.

[18] W. D. Neumann, Complex algebraic plane curves via their links at infinity, Invent. Math. 98 (1989), no. 3, 445-489

[19] _ Irregular links at infinity of complex affine plane curves, Quart. J. Math. Oxford Ser. (2) 50 (1999), no. 199, 301-320. 
[20] W. D. Neumann and P. G. Wightwick, Algorithms for polynomials in two variables, Combinatorial and computational algebra (Hong Kong, 1999), Contemp. Math., vol. 264, Amer. Math. Soc., Providence, RI, 2000, pp. 219-235.

[21] I. Newton, Curves by Sir Isaac Newton, Lexicum technicum: An universal dictionary or arts and sciences. Vol. II (J. Harris, ed.), London, 1710; reprinted in The mathematical works of Isaac Newton. Vol. II, with an introduction by D. T. Whiteside, Johnson Reprint Corp., New York, 1967, pp. 135-161.

[22] H. Pinkham, Courbe planes ayant une seule place à l'infini, Séminaire sur les Singularités des Surfaces (Centre de Mathématiques de l'École Polytechnique, Palaiseau, 1977-1978).

[23] M. Puiseux, Recherches sur les fonctions algébriques, Journal de Math. (1) 15 (1850), 365-480.

[24] A. Sathaye, Degree-semigroups of plane algebraic curves, preprint.

[25] A. Sathaye and J. Stenerson, Plane polynomial curves, Algebraic geometry and its applications (West Lafayette, IN, 1990), Springer, New York, 1994, pp. 121-142.

[26] A. Schinzel, Polynomials with special regard to reducibility, with an appendix by U. Zannier, Encyclopedia of Mathematics and its Applications, vol. 77, Cambridge University Press, Cambridge, 2000.

[27] M. Suzuki, Propriétés topologiques des polynômes de deux variables complexes, et automorphismes algébriques de l'espace $\mathbf{C}^{2}$, J. Math. Soc. Japan 26 (1974), 241-257.

[28] _ Affine plane curves with one place at infinity, Ann. Inst. Fourier (Grenoble) 49 (1999), no. 2, 375-404

[29] E. W. von Tschirnhausen, Acta eruditorum, Leipzig, 1683.

[30] R. J. Walker, Algebraic curves, Dover Publications Inc., New York, 1962.

[31] P. G. Wightwick, Algebraic curves and their links, Minor Masters Thesis, University of Melbourne, 1995.

[32] _ Equivalence of polynomials under automorphisms of $\mathbb{C}^{2}$, J. Pure Appl. Algebra 157 (2001), no. 2-3, 341-367.

[33] M. G. Zal̆denberg and V. Ya. Lin, An irreducible, simply connected algebraic curve in $\mathbf{C}^{2}$ is equivalent to a quasihomogeneous curve, Dokl. Akad. Nauk SSSR 271 (1983), no. 5, 1048-1052 (Russian) 